Der Medizinischen Fakultät der Georg-August-Universität Göttingen Eingereicht von Prof. Dr. med. F. Hufert

\title{
The Role of NS3 Helicase Domain in Hepatitis C Virus Particle Assembly
}

\author{
INAUGURAL-DISSERTATION \\ zur Erlangung des Doktorgrades \\ der Medizinischen Fakultät \\ der Georg-August-Universität zu Göttingen
}

vorgelegt von

Caroline Bouter

aus

Peine

Göttingen 2012 
Diese Dissertation wurde erstellt an der

\section{Yale SCHOOL OF MEDICINE}

295 Congress Avenue, New Haven, CT, USA im Jahr 2010 unter der Betreuung von Prof. Brett Lindenbach (PhD).

Dekan:

Prof. Dr. med. M. Schön

1. Berichterstatter: $\quad$ Prof. Dr. med. F. Hufert

2. Berichterstatter/in:

3. Berichterstatter/in:

Tag der mündlichen Prüfung: 


\section{Table of Contents}

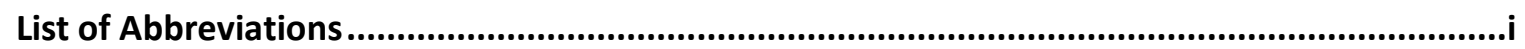

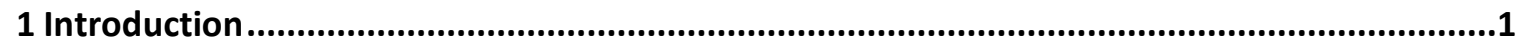

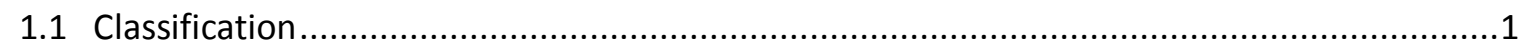

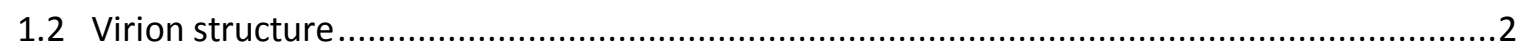

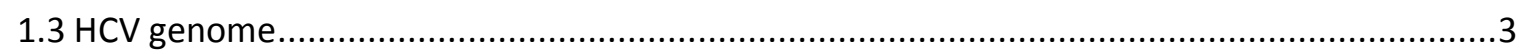

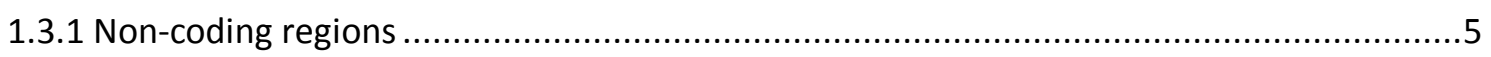

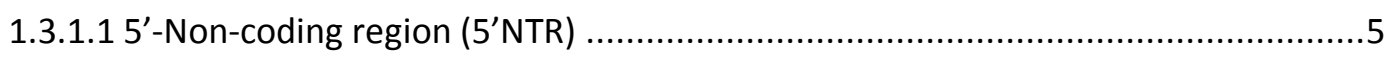

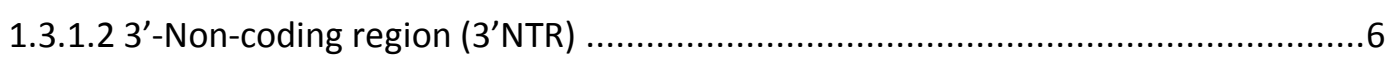

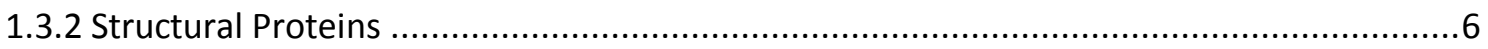

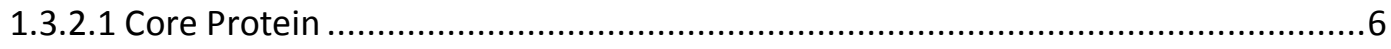

1.3.2.2 Envelope Glycoproteins (E1 and E2) .........................................................

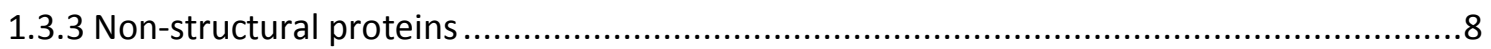

1.3.3.1 p7

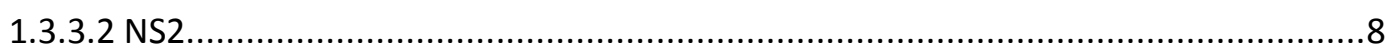

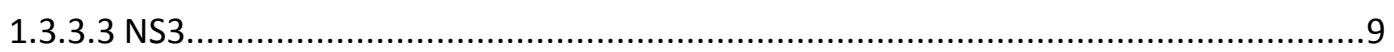

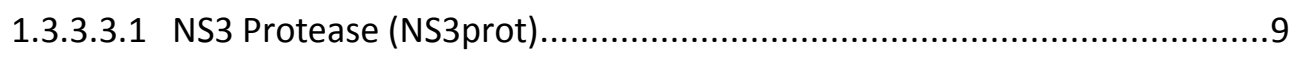

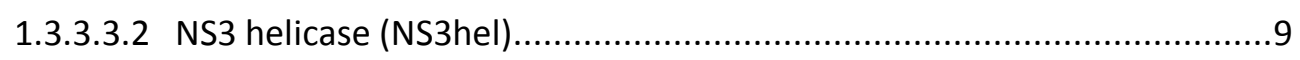

1.3.3.3.3 NS3hel structure .......................................................................10

1.3.3.3.4 Mechanism of unwinding duplex DNA or RNA....................................12

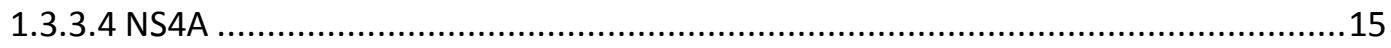

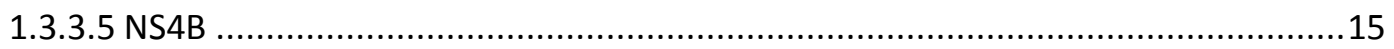

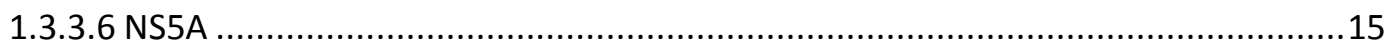

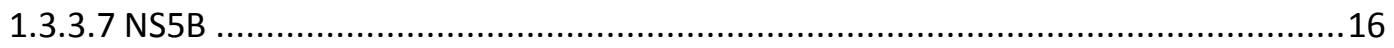

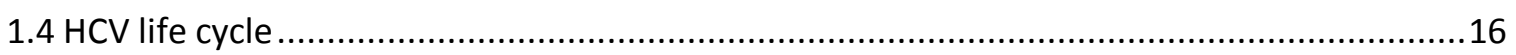

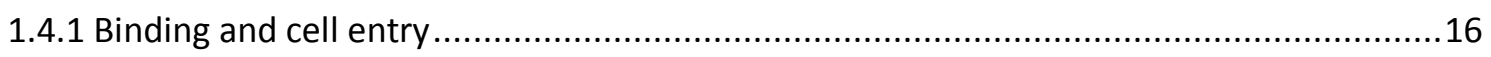

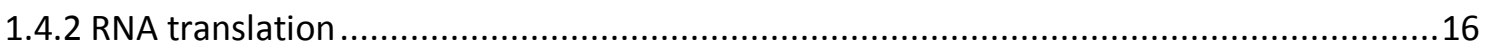

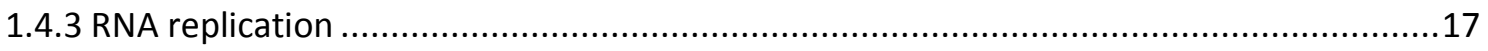

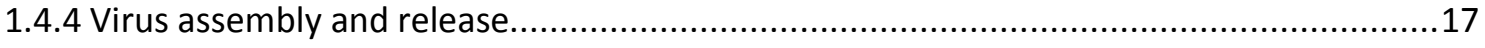

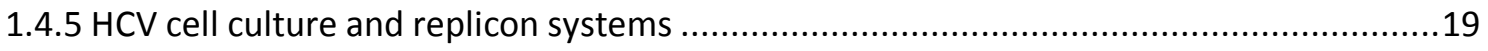

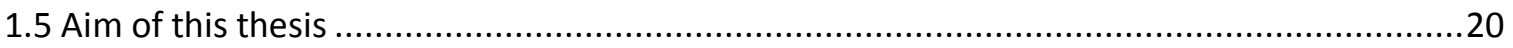




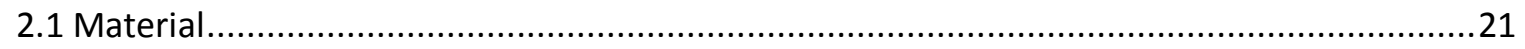

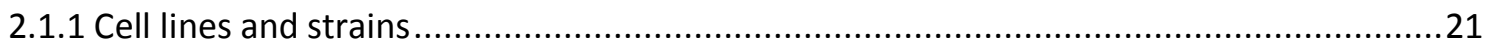

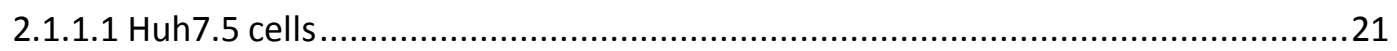

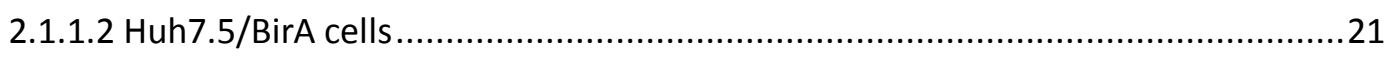

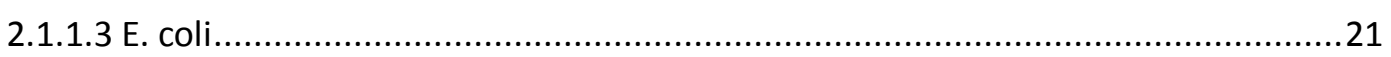

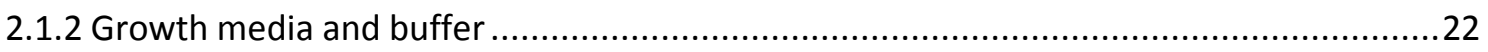

2.1.2.1 Media for Escherichia coli cultures..................................................22

2.1.2.2 Pulldown experiment \& Western blot buffer ........................................23

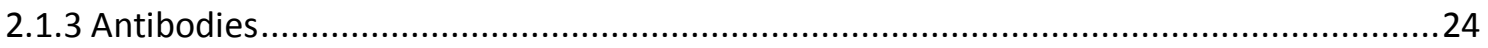

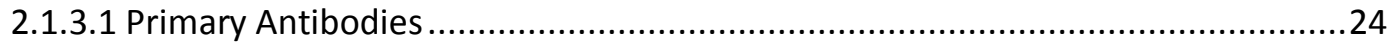

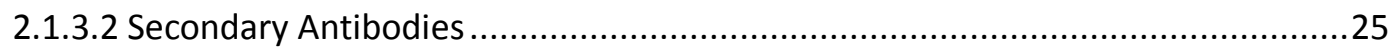

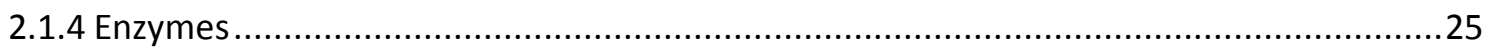

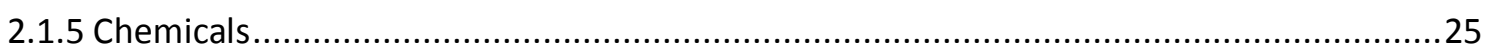

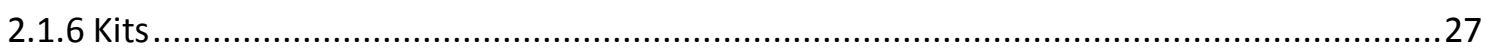

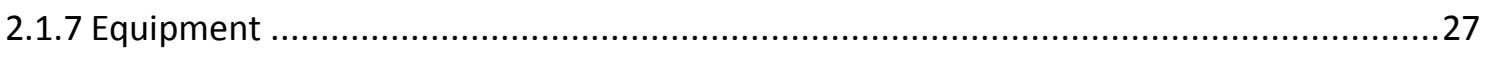

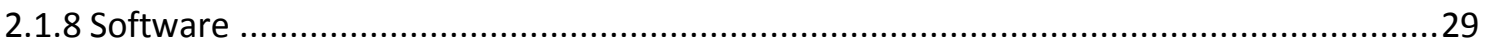

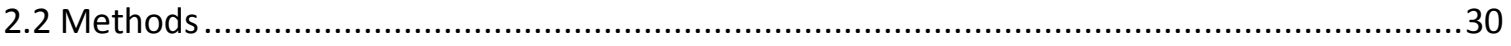

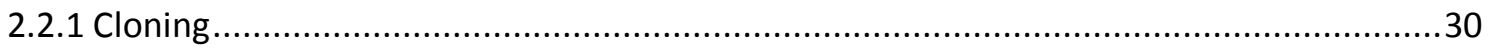

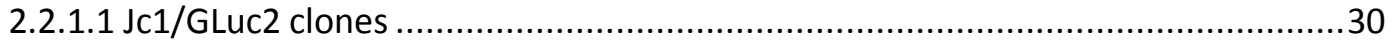

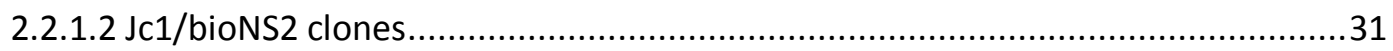

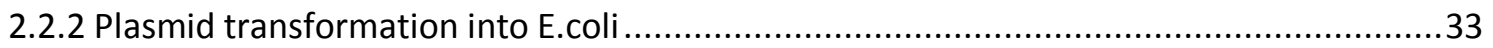

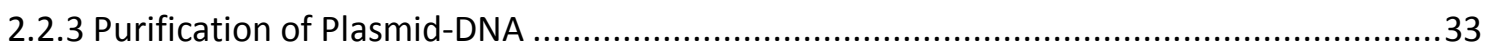

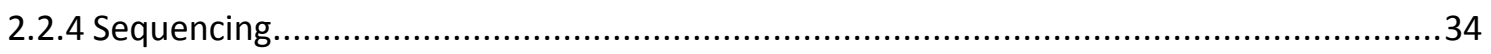

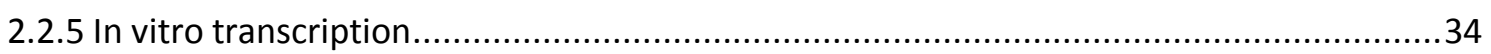

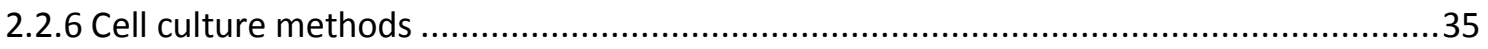

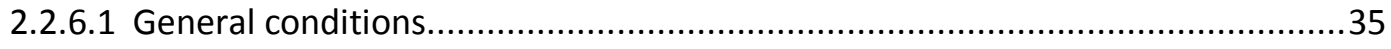

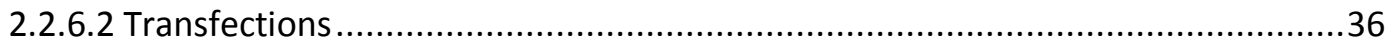

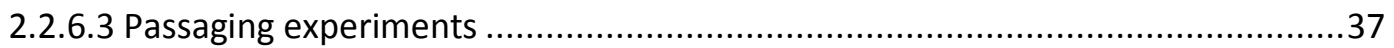

2.2.6.4 GLuc activity/Luciferase assay........................................................38

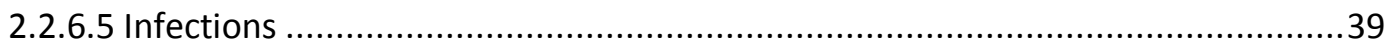

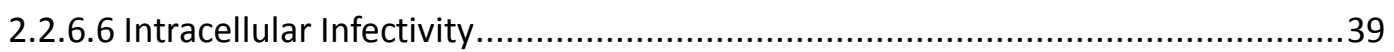

2.2.6.7 Anti-NS5a staining of HCV-infected Huh 7.5 cells .......................................40

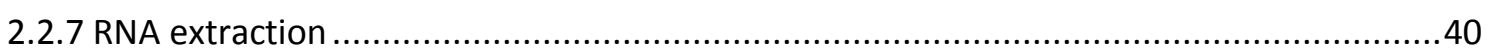




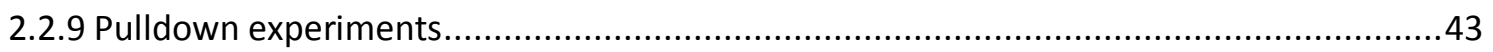

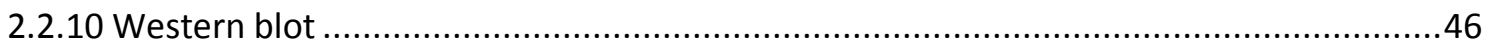

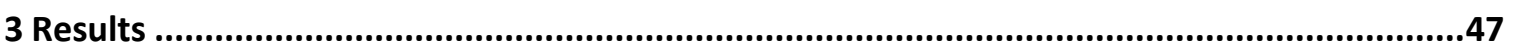

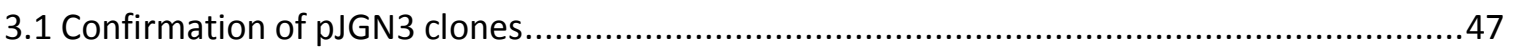

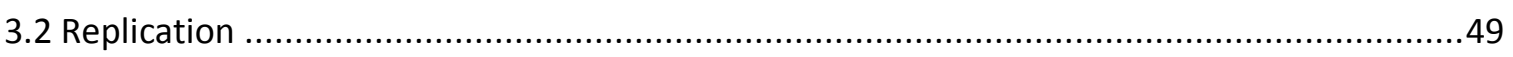

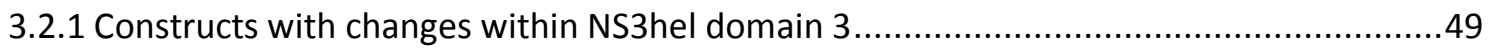

3.2.1.1 Replication of constructs with changes within NS3hel domain 3 alpha helix 20 and 21

3.2.1.2 Replication of constructs with changes within NS3hel domain 3 alpha helix 14,

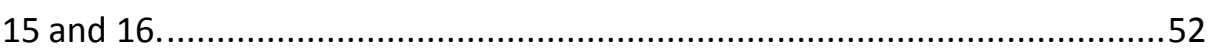

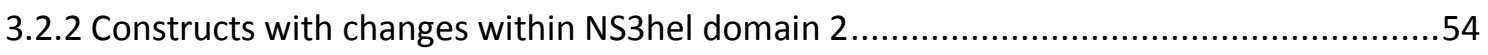

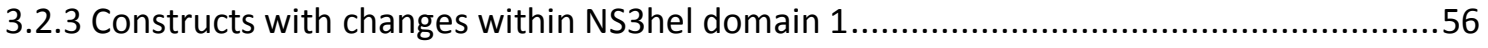

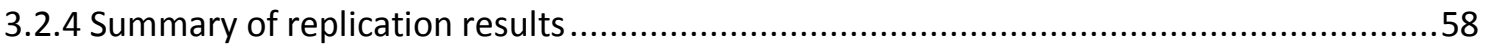

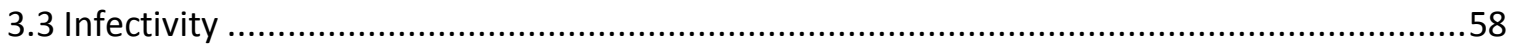

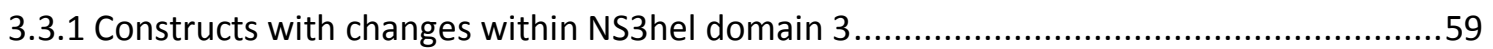

3.3.1.1 Infectivity of constructs with changes within NS3hel domain 3 alpha helix 20

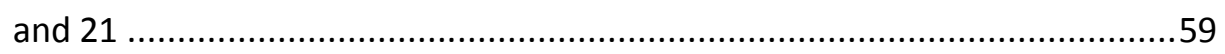

3.3.1.2 Infectivity of constructs with changes within NS3hel domain 3 alpha helix 14,

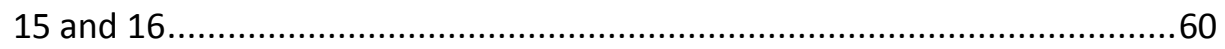

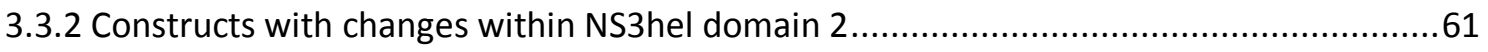

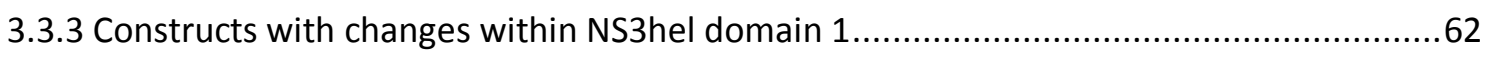

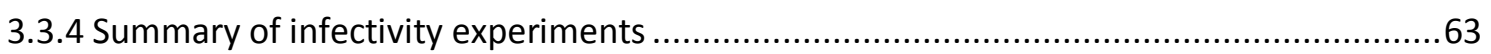

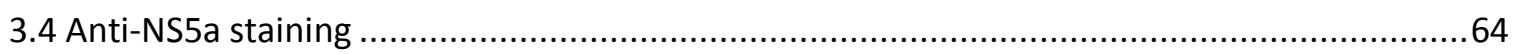

3.5 Studying HCV virus particle assembly: JGN3-2, JGN3-12 and JGN3-24 - constructs that were able to replicate but not to be infectious .........................................................66

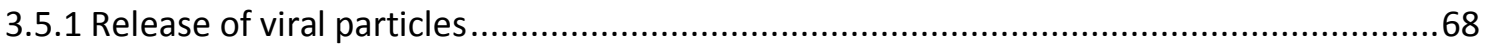

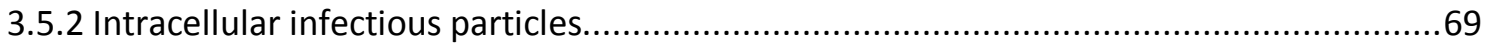

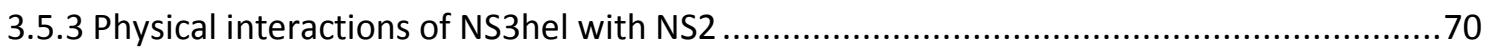

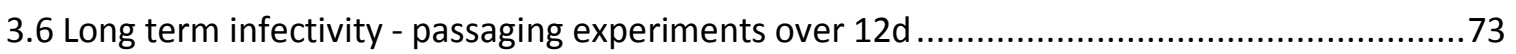

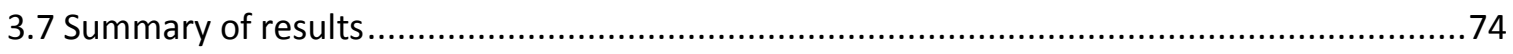

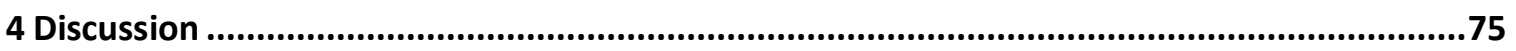

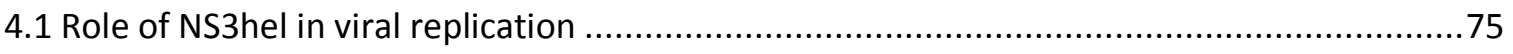

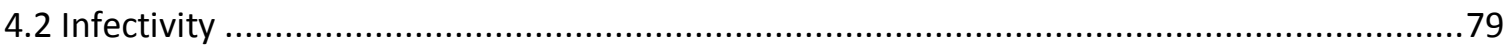

4.3 JGN3-2, JGN3-12 and JGN3-24 - constructs that were able to replicate but not to be infectious 
4.3.1 NS3hel and virus particle assembly......

4.3.2 How does NS3hel contribute to virus particle assembly? .............................................82

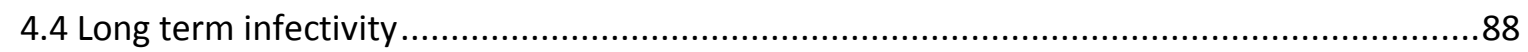

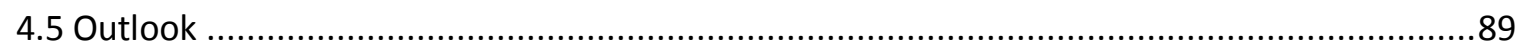

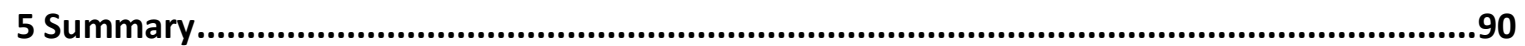

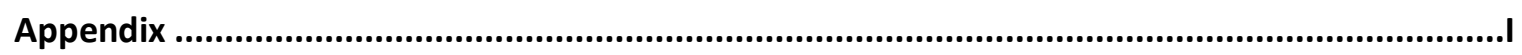

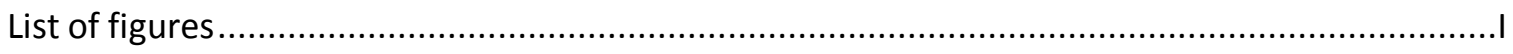

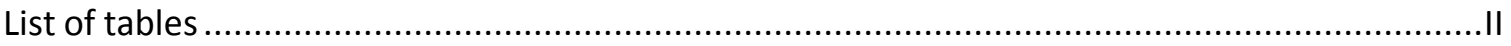

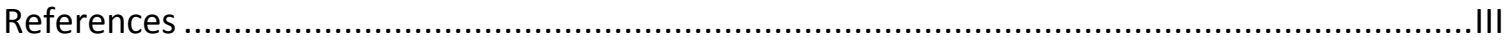




\section{List of Abbreviations}

\begin{tabular}{|c|c|}
\hline Abbreviation & Long version \\
\hline ARF & Alternative reading frame protein \\
\hline ATP & Adenosine triphosphate \\
\hline BAP & Biotin acceptor peptide \\
\hline Biotin-AP & Biotin-acceptor peptide \\
\hline BSA & Bovine serum albumine \\
\hline DMEM & Dulbecco's modified Eagle medium \\
\hline DNA & Deoxyribonucleic acid \\
\hline DPBS & Dulbecco's phosphate-buffered saline \\
\hline DTT & Dithiothreitol \\
\hline E.coli & Escherichia coli \\
\hline EM & Electron microscope \\
\hline ER & Endoplasmic reticulum \\
\hline $\mathrm{EtBr}$ & Ethidium bromide \\
\hline FCS & Fetal calf serum \\
\hline GLuC & Gaussia Luciferase \\
\hline GTPase & Guanine triphosphatase \\
\hline $\mathrm{HCV}$ & Hepatitis $\mathrm{C}$ virus \\
\hline HCVpp & Hepatitis C virus pseudo particles \\
\hline IFN & Interferon \\
\hline IRES & Internal ribosome entry site \\
\hline LB & Lysogeny broth \\
\hline LD & Lipid droplet \\
\hline mRNA & Messenger RNA \\
\hline NF-kB & $\begin{array}{l}\text { Nuclear factor 'kappa-light-chain-enhancer' of } \\
\text { activated B-cells }\end{array}$ \\
\hline NS & Non-structural protein \\
\hline NTP & Nucleoside triphosphate \\
\hline NTR & Non-coding regions \\
\hline PBS & Phosphate buffered saline \\
\hline PKR & Protein kinase $\mathrm{R}$ \\
\hline qRT-PCR & $\begin{array}{l}\text { Quantitative reverse transcription polymerase } \\
\text { chain reaction }\end{array}$ \\
\hline $\mathrm{RdRp}$ & RNA-dependent RNA polymerase \\
\hline $\mathrm{RF}$ & Replicative form \\
\hline $\mathrm{RI}$ & Replicative intermediates \\
\hline RLU & Relative light units \\
\hline RNA & Ribonucleic acid \\
\hline RNAP & RNA polymerase \\
\hline SDS & Sodium dodecylsulfate \\
\hline SDS-PAGE & $\begin{array}{l}\text { Sodium dodecylsulfate polyacrylamide } \\
\text { electrophoresis }\end{array}$ \\
\hline SL & Stem-loop \\
\hline SOB & Super optimal broth \\
\hline SR-BI & Scavenger receptor class B type I \\
\hline
\end{tabular}




\begin{tabular}{ll}
\hline SSRNA & Single-stranded ribonucleic acid \\
\hline TAE & Tris-acetate-EDTA \\
\hline TBS & Tris-buffered saline \\
\hline TNF & Tumor necrosis factor \\
\hline WHO & World Health Organization \\
\hline WT & Wild type \\
\hline
\end{tabular}




\section{Introduction}

Hepatitis C, which was identified in 1989 as non-A, non-B hepatitis, represents an immense global health problem. According to the World Health Organization (WHO) 130-170 million people are infected worldwide which is around 3\% of the world's population. Over 350000 infected people die from hepatitis C virus (HCV)-related diseases every year.

The liver is the major site of HCV infection. Hepatitis $C$ causes acute and chronic liver disease which can lead to cirrhosis, liver failure and hepatocellular carcinoma. Furthermore, different studies detected HCV RNA in cells other than hepatocytes (Lemon et al. 2007). Evidence for extrahepatic replication was found in the central nervous system, lymphoid cells and gut epithelial cells (Lindenbach et al. 2007). Though, the relevance of these findings remains uncertain and controversial.

Current HCV therapies have to be improved as standard therapy with a combination of ribavarin and pegylated interferon- $\alpha$ is ineffective. It permanently eliminates detectable virus in only around $40 \%$ of genotype 1 infected patients and around $70-80 \%$ of patients infected with genotype 2 (Manns et al. 2001). The therapy is often poorly tolerated and provides many side effects. No primary preventions, no vaccines and no post-exposure prophylaxis were developed so far (Lemon et al. 2007). Studying the hepatitis $C$ virus life cycle can reveal new approaches for the development of vaccines or new antiviral therapies. Different steps in the $\mathrm{HCV}$ life cycle are possible targets for new therapeutic drugs.

\subsection{Classification}

The hepatitis $C$ virus is an enveloped single-stranded RNA virus with positive polarity (ss(+)RNA). It is a member of the Flaviviridae family and classified in the Hepacivirus genus (Choo et al. 1991).

HCV shows extraordinary genetic variability due to its abundant virus production rate, high error rate and lack of proofreading activity of the RNA-dependent RNA polymerase (RdRp) (Pawlotsky 2003). Based on the genetic differences six major genotypes could be determined. These variants differ up to $30 \%$ in their sequence over the entire genome. The six genotypes can be further divided into several subtypes. Minor sequence variations, around $1-2 \%$, are common. These so called 'quasispecies' co-circulate within single infected individuals. Genetic relationship between genotypes and regions of distribution are shown in Figure 1 (Simmonds 2004). 


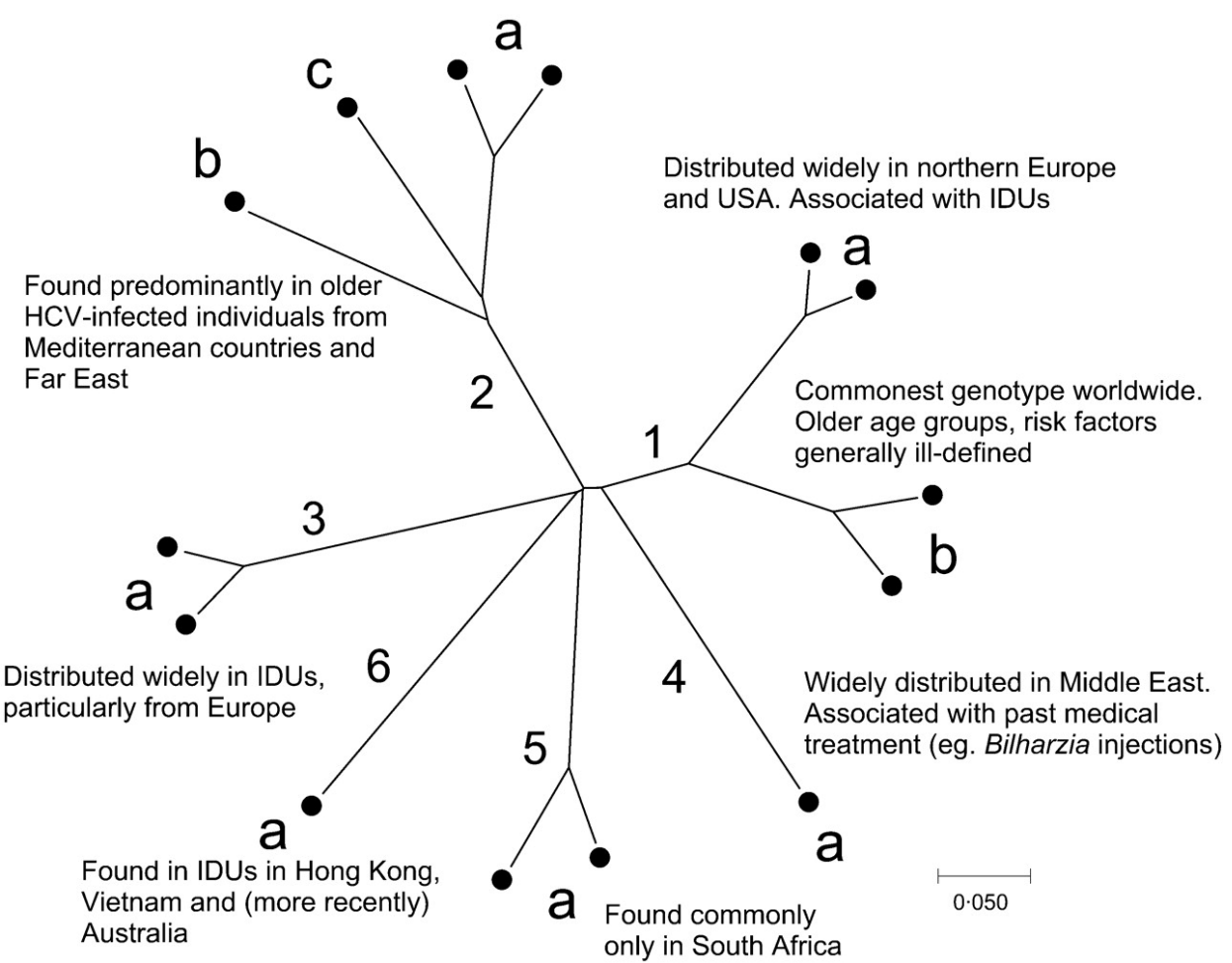

Figure 1 Phylogenetic tree of major HCV genotypes and subtypes (Simmonds 2004 p. 3176). Evolutionary distances and regions of distribution are shown.

\subsection{Virion structure}

$\mathrm{HCV}$ particles are described by filtration and electron microscope (EM) techniques to be between 30 and $80 \mathrm{~nm}$ in diameter (He et al. 1987; Yuasa et al. 1991). EM images show spherical virions containing an electron dense core surrounded by a lipid envelope (Wakita et al. 2005). HCV RNA is thought to be encapsidated by core proteins surrounded by a lipid layer including HCV glycoproteins E1 and E2. A model of the HCV virion is shown in figure 2A. Furthermore, HCV shows an unusually low buoyant density between 1.03 to $1.10 \mathrm{~g} / \mathrm{ml}$ (Bradley et al. 1991; Hijikata et al. 1993). This is likely explained by its described interactions with lipoproteins (Lindenbach et al. 2007). 
A

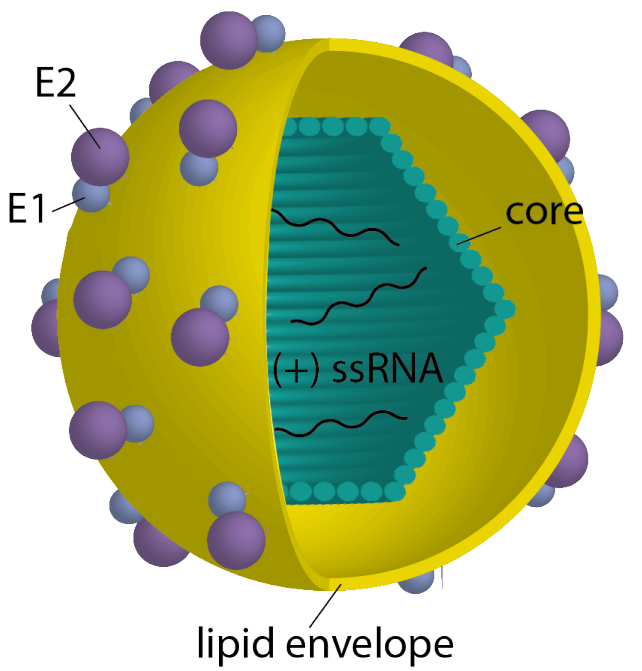

B

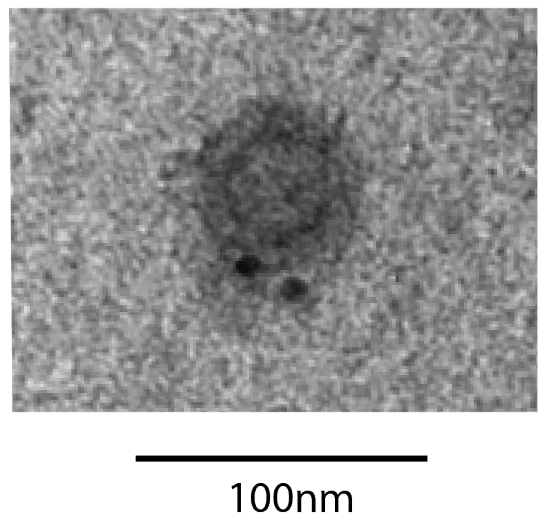

Figure 2 HCV virion structure. (A) Model of a HCV virion. A lipid envelope containing HCV glycoproteins E1 and E2 surrounds the core-encapsidated viral genome. (B) EM image of a HCV virion (Wakita et al. 2005 p.793). Particle was detected by immunogold labeling for E2.

\subsection{HCV genome}

The HCV genome is uncapped and about $9.6 \mathrm{~kb}$ in size (Lindenbach et al. 2007).

The genome is coding for one large polyprotein (about 3011 amino acids in size) that is proteolytically cleaved into ten viral proteins. The polyprotein is flanked by $\mathrm{N}$ and $\mathrm{C}$ terminal non-coding regions (NTRs or NCRs). The 5' NTR includes an internal ribosome entry site (IRES) which mediates polyprotein translation (Wang et al. 1993). Furthermore both NTRs are required for viral replication (Wang et al. 1993; Friebe et al. 2001).

Proteins can be divided into structural and non-structural proteins. Amino terminal one third of the polyprotein includes the viral structural proteins core protein $(C)$ and envelope glycoproteins E1 and E2. Between structural and non-structural HCV proteins a small integral membrane protein $(p 7)$ is found. The actual function of $p 7$ as a structural or nonstructural protein is unknown. The remainder of the polyprotein contains the non-structural proteins NS2, NS3, NS4A, NS4B, NS5A and NS5B which coordinate the viral life cycle (Bartenschlager et al. 2004).

Next to these proteins at least three different forms of alternative reading frame proteins (ARF) or frameshift $(F)$ proteins are described. Translation of this protein is also initiated at the core gene start codon and alternative proteins are produced through frameshifting. These proteins might play a role in the HCV life cycle which is unknown so far (Xu 2001). 


\begin{tabular}{|c|c|c|c|}
\hline HCV protein & Size [kDa] & Main function & Source \\
\hline Core & $\begin{array}{l}21 \text { (mature } \\
\text { form) }\end{array}$ & Nucleocapsid protein, RNA binding & $\begin{array}{l}\text { Lindenbach et al. } \\
2007\end{array}$ \\
\hline E1 & 30 & Envelope protein & $\begin{array}{l}\text { Lindenbach et al. } \\
2007\end{array}$ \\
\hline E2 & 70 & Envelope protein & $\begin{array}{l}\text { Lindenbach et al. } \\
2007\end{array}$ \\
\hline p7 & 7 & Unknown, probably ion channel & $\begin{array}{l}\text { Lindenbach et al. } \\
2007\end{array}$ \\
\hline NS2 & 23 & NS2-NS3 proteinase, assembly & $\begin{array}{l}\text { Lindenbach et al. } \\
2007\end{array}$ \\
\hline NS3 & 70 & $\begin{array}{l}\text { NS2-NS3 proteinase, NS3-NS4A } \\
\text { proteinase, helicase, NTPase, RNA } \\
\text { binding, assembly? }\end{array}$ & $\begin{array}{l}\text { Lindenbach et al. } \\
2007\end{array}$ \\
\hline NS4A & 8 & Co-factor NS3-NS4a proteinase & $\begin{array}{l}\text { Lindenbach et al. } \\
2007\end{array}$ \\
\hline NS4B & 27 & $\begin{array}{l}\text { Integral membrane protein, organizing } \\
\text { membrane-bound replication complex, } \\
\text { phosphorylation NS5A }\end{array}$ & $\begin{array}{l}\text { Lindenbach et al. } \\
2007\end{array}$ \\
\hline NS5A & 58 & Replication & $\begin{array}{l}\text { Lindenbach et al. } \\
2007\end{array}$ \\
\hline NS5B & 68 & $\begin{array}{l}\text { Major enzyme of RNA replication (RNA } \\
\text { dependent RNA polymerase) }\end{array}$ & $\begin{array}{l}\text { Lindenbach et al. } \\
2007\end{array}$ \\
\hline ARF proteins & Unknown & Unknown & Xu, 2001 \\
\hline
\end{tabular}

Table 1 Structural and non-structural HCV proteins.

The polyprotein is co-and posttranslational cleaved by viral and host cell proteases. The region between core and NS2 is processed by the host cell signal peptidase. Maturation of core protein requires releasing its association to the $\mathrm{E} 1$ signal peptide. This is performed by the signal peptide peptidase. NS2 and NS3 are separated by NS2-NS3 cysteine autoprotease cleavage. The remainder of the polyprotein is processed by NS3-NS4A serine protease cleavage (Bartenschlager et al. 2004). 
A

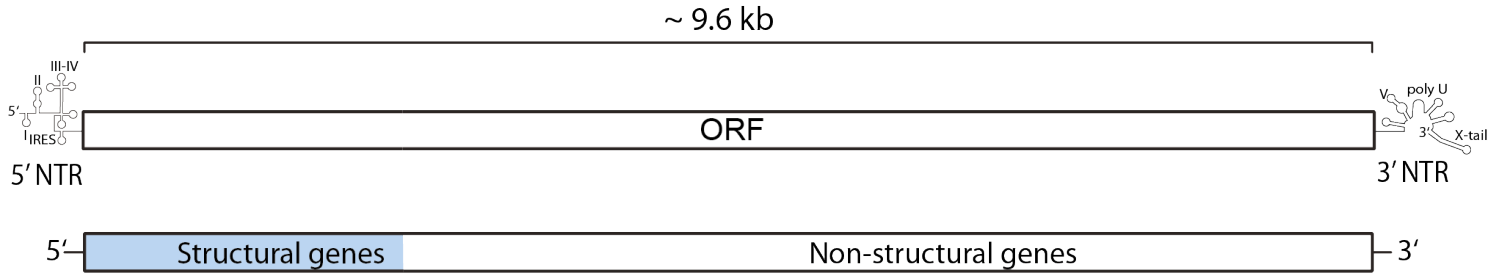

Translation

B

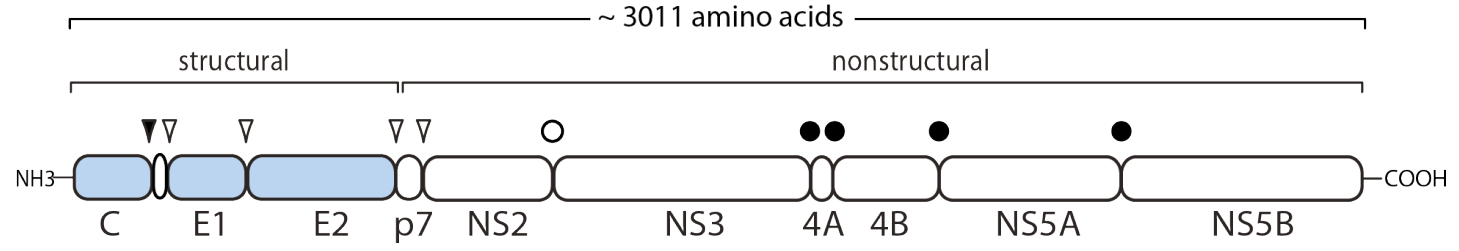

C

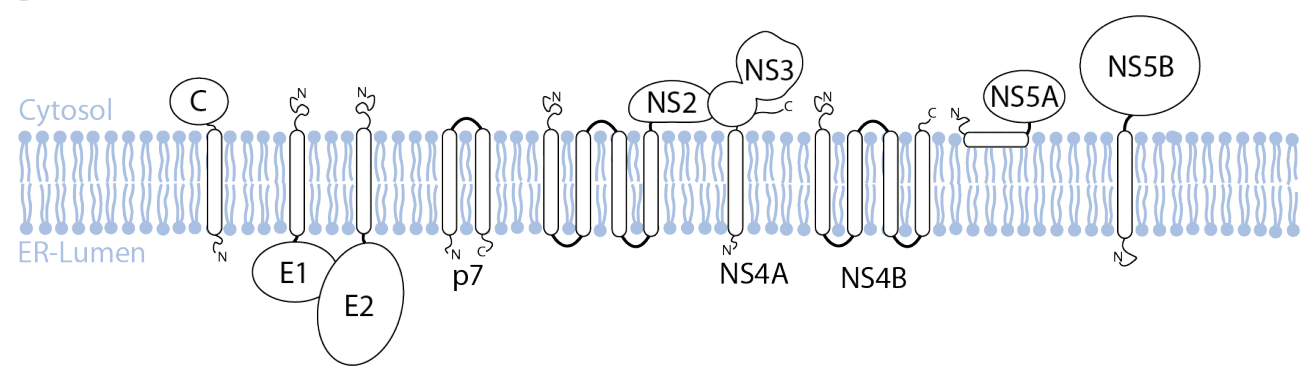

Figure $3 \mathrm{HCV}$ genome structure and polyprotein processing. (A) Genome structure of HCV. The open reading frame (ORF) is flanked by non-coding regions (NTR). The genome is lacking a cap-structure. Internal ribosome entry site (IRES) is located within the $5^{\prime}$ non-coding region. Four major domains (I-IV) of the $5^{\prime}$ NTR are labeled. The $3^{\prime}$ non-coding region includes a variable region (V), a poly $\mathrm{U}$ part and a conserved region (X-tail). (B) Translation produces a polyprotein of about 3011 amino acid in size. Polyprotein is co-and post-translational processed into 10 proteins (Frameshift leads to an alternative $11^{\text {th }}$ protein). Structural proteins are highlighted in blue. Cleavage sites are indicated by arrowheads and circles. Signal peptidase (SP) cleavage sites are marked by open arrowheads, signal peptide peptidase (SPP) cleavage by a filled arrowhead, NS2-NS3 cysteine autoprotease cleavage by an open circle and NS3-NS4 serine protease cleavage by filled circles. (C) Membrane topology of HCV proteins. Core is show pre-SPP-cleavage and still in its membrane anchored form. E1 and E2 are shown post-SP-cleavage and after reorientation of the C-termini. Further details are found in the text (Lindenbach et al. 2007).

\subsubsection{Non-coding regions}

\subsubsection{5'-Non-coding region (5'NTR)}

The 5'NTR is a well conserved, 341 nucleotide long RNA sequence. Among HCV genotypes sequence identicalness of the 5' non-translating region is more than 90\% (Shi and Lai 2006). 
The secondary and tertiary structure is quite complex and consists of four domains (I-IV) and a pseudoknot $(\Psi)$ (Wang et al. 1993). It does not contain a 5' methyl cap structure. Domain I, the first 40 nucleotides of 5 'NTR, is important for replication, similar to other positive ssRNA viruses. The remainder of the $5^{\prime}$ NTR is essential for translation. It includes the internal ribosome entry site (IRES). IRES also overlaps first nucleotides of the HCV coding region though it is not clear whether it is a functional component of IRES or only interacts with it by stabilizing the complex secondary structure (Krekulová et al. 2006; Reynolds et al. 1995). IRES coordinates the cap-independent translation of the HCV genome (Shi and Lai 2006).

\subsubsection{3'-Non-coding region (3'NTR)}

3'NTR follows the UGA termination codon of the coding region. Length of 3'NTR varies between 200 and $235 \mathrm{nt}$. It consists of a short variable region of about $40 \mathrm{nt}$, a polyuridine/polypyrimidine (poly(U/UC) tract of a heterogeneous length and a highly conserved 3'X domain of 98 nt (X-tail) (Krekulová et al. 2006). Mutagenesis studies of Friebe et al. (2001) mapped regions of the 3'NTR which are important for replication. These studies revealed that the X-tail is especially important for replication. The poly(U/UC) tract needs to be at least $26 \mathrm{nt}$ long to allow replication. Deletion of the variable region also reduces replication significantly. Studies of You and Rice (2008) revealed that RNA replication requires a functional long distance kissing-loop structure between stem-loop 2 (SL2) of the 3' $X$-tail and an element of NS5B within the coding region. This could explain why a short poly(U/UC)-tract disables replication as the poly(U/UC) could function as a spacer for these long distance base-pairing interactions. However, the actual function of the kissing interaction remains to be determined (You and Rice 2008).

\subsubsection{Structural Proteins}

\subsubsection{Core Protein}

Core protein is a highly conserved protein which plays an important role in virus particle assembly. It is characterized to form the main component of the HCV nucleocapsid. As mentioned above core protein is processed in two steps. Host signal peptidase removes an immature membrane anchored form of core (191 aa) from the polyprotein. Further cleavage of the signal peptide peptidase within the membrane anchor between amino acid 173 and 182 releases the 21 kDa mature core protein (Krekulová et al. 2006; Dubuisson 2007; Lindenbach et al. 2007).

After processing the mature form of the core protein can be separated into two domains: an 122 aa N-terminal hydrophilic domain and a C-terminal hydrophobic domain of around 50 aa (Krekulová et al. 2006). Domain I can bind RNA unspecifically. It is shown that interaction of 
the N-terminal part of domain I with the 5 'NCR can influence IRES function resulting in downregulation of translation (Honda et al. 1996). Domain II is involved in proper folding of domain I and mediates the binding of core to lipid droplets and membranes (Lindenbach et al. 2007). E1 has been shown to interact with core which seems to be necessary for particle assembly (Lo et al. 1995). Core protein also assist in proper folding of E1 (Merola et al. 2001).

The mature form of the protein multimerizes and binds the HCV genome forming the viral nucleocapsid in virus particle assembly.

Next to this structural function core is found to additionally influence different cellular pathways of the host cell like transcription, apoptosis, lipid metabolism and immune response (McLauchlan 2000). A likely explanation of these functions is the interaction of core with host cell proteins. Furthermore, core can bind lipid droplets inducing steatosis in transgenic mice with over-expressed core protein. Interaction with TNF, Fas, NF-KB most probably influences immune response and apoptosis. These reactions might be important in the development of chronic infections (Krekulová et al. 2006).

Furthermore core protein is described to control gene expression by binding human DEAD box protein 3 (DDX3) from the DEAD-box RNA helicase family. This might influence transcription, pre-mRNA splicing and other reactions of RNA metabolism of the host cell (Owsianka and Patel 1999).

\subsubsection{Envelope Glycoproteins (E1 and E2)}

HCV envelope glycoproteins E1 and E2 are type-I transmembrane proteins. The about 30 $\mathrm{kDa}(\mathrm{E} 1)$ and $70 \mathrm{kDa}(\mathrm{E} 2)$ proteins contain a large N-terminal extracellular domain and a Cterminal transmembrane domain. E1 and E2 are essential for virus entry. They are likely to mediate virus particle attachment and membrane fusion. They also participate in virion assembly (Dubuisson 2007). Extracellular functional units of E1 and E2 are suggested as non-covalent E1-E2 heterodimers, which are highly glycosylated (Bartosch and Cosset 2006). The E1-E2 heterodimers are suggested to function as a ligand for cellular receptors. Several potential receptors could be identified, from which CD81 tetraspanin and scavenger receptor class $\mathrm{B}$ type I (SR-BI) could be determined to play a role in HCV entry (Cocquerel et al. 2006). Transmembrane domains are thought to function as membrane anchors and also as signal peptides influencing localization and heterodimerization of the extracellular domains (Bartosch, Cosset 2006). 


\subsubsection{Non-structural proteins}

\subsubsection{1 p7}

p7 is a small integral membrane protein. It is believed that it spans the membrane twice with both $\mathrm{N}$ - and C-termini facing the ER lumen (Carrere-Kremer et al. 2002). It is suggested to function as an ion-channel, but the role of $p 7$ in the viral life cycle remains unclear. It is also unclear if it is a structural or a non-structural protein. It is predicted that $p 7$ is not a component of the virion (Jones CT et al. 2007). Protein p7 could be localized in the ER, mitochondria and the plasma membrane of hepatocytes (Carrere-Kremer et al. 2002). It is referred to be able to multimerize in vitro forming membrane calcium ion channels which can be blocked by amantadine (Griffin et al. 2003). Sakai et al. (2003) showed that $p 7$ is essential for infectivity in vivo. Work with subgenomic replicons which do not include p7 revealed that it is not necessary for HCV RNA replication (Lohmann et al. 1999). The role of $p 7$ remains unclear to date, but one can assume that it might play a role in viral entry and release alike similar viral transmembrane channels in other viruses (Lindenbach et al. 2007).

\subsubsection{NS2}

NS2 is an integral polytopic membrane protein. The actual topology of NS2 is not clear yet, but it is suggested that it spans the membrane four times with $\mathrm{N}$-and $\mathrm{C}$-termini facing the ER lumen (Yamaga and Ou 2002). The $\mathrm{N}$-terminus of NS2 is cleaved from $\mathrm{p} 7$ by a signal peptide peptidase within the ER. The C-terminal domain functions as cysteine protease which requires the $\mathrm{N}$-terminal protease domain of NS3 (first 180 residues). It is suggested that NS3 is needed to stabilize protein folding (Welbourn and Pause 2007). The NS2/3autoprotease cleaves the NS2/NS3 junction in an independent manner from NS3/NS4 protease activity (Lindenbach et al. 2007).

For correct function of the protease the coordination of a zinc molecule by NS3 residues 1123, 1127, 1171 and 1175 are needed to stabilize NS2/3 structure (Welbourn and Pause 2007). As the NS2/3 protease cleavage takes place in the cytosolic space, it is suggested that the NS2/NS3 region has to be re-orientated after NS2/3 cleavage (Dubuisson 2007). Furthermore, recent data also reveals that NS2 plays a role in virus particle assembly. It is also suggested that it is involved in particle release. But its precise role in these processes is unknown to date. The Lindenbach laboratory as well as other groups recently published protein-protein interactions between NS2 and other HCV proteins which might be essential for virus particle assembly (Stapleford and Lindenbach 2011; Ma et al. 2011). It is suggested that NS2 organizes particle assembly by these protein-protein interactions recruiting HCV protein complexes to sites of assembly reactions close to lipid droplets (Jirasko et al. 2010). 


\subsubsection{NS3}

The HCV NS3 is a multifunctional protein which is around $70 \mathrm{kDa}$ in size. The N-terminal one third contains a serine protease and the C-terminal two thirds contain a RNA helicase. The protease processes the HCV polyproteins non-structural region and also some cellular proteins. The exact function of the helicase remains unclear so far.

\subsection{NS3 Protease (NS3prot)}

The serine protease domain of NS3 belongs to the superfamily of trypsin/chemotrypsin proteases. NS3 protease is responsible for cleavage of the HCV polyprotein at the NS3/4A, $\mathrm{NS} 4 \mathrm{~A} / 4 \mathrm{~B}, \mathrm{NS} 4 \mathrm{~B} / 5 \mathrm{~A}$ and $\mathrm{NS} 5 \mathrm{~A} / 5 \mathrm{~B}$ sites (Figure 3 ). In addition to its role in polyprotein processing NS3 protease is also involved in proteolytic cleavage of host cell proteins which are involved in intracellular viral responses. This causes decreased expression of multiple host defense genes contributing to the development of persistent infections (Li et al. 2005). The structure of NS3 protease reveals two $\beta$-barrel domains adjoined by two small $\alpha$-helices. Substrate binding is located in a cleft between the $\beta$-barrel domains. Interaction with NS4A is required for complete folding and activity of the NS3 serine protease. It also contributes in positioning of the catalytic triad and the substrate (Barbato et al. 1999). Next to stabilizing the proteins conformation NS4A is told to serve as a membrane anchor with its $\mathrm{N}$-terminal hydrophobic domain (Wölk et al. 2000). Furthermore a $\mathrm{Zn}^{2+}$ ion is required to stabilize the structure. $\mathrm{Zn}^{2+}$ is coordinated by three cysteine residues and one histidine (De Francesco et al. 1996).

The cleavage mechanism of the NS3 protease is identical to the one of other serine proteases. A catalytic triad is found in the active site of NS3 protease. It consist of three amino acids, Ser-139, His-57 and Aps-81 which are essential for catalysis of peptide cleavage. Substrate binding leads to ordered reactions of the peptide cleave mechanism. Several intermediates are generated releasing the polypeptide bond of the substrate by hydrolysis (Raney et al. 2010).

\subsection{NS3 helicase (NS3hel)}

Helicases are proteins that separate double-stranded nucleic acids into single-strands in an NTP-dependent reaction. Helicases are involved in several cellular functions such as premRNA splicing, ribosome assembly, translation initiation and other cellular RNA transactions (Tai et al. 1996; Jankowsky and Fairman 2007). Helicases can be divided into five major superfamilies (SF 1-5) based on sequence homology (Gorbalenya and Koonin 1993). SF-2 is further divided into several subgroups (DEAD, DExH, etc). NS3 helicase (NS3hel) domain is a superfamily 2 (SF-2) DExH helicase/NTPase. Other members of SF-2 DExH helicases 
include Yellow fever virus NS3, Dengue fever virus NS3 and Vaccina virus NPH-II (Jankowsky 2010). SF-1 and SF-2 proteins are characterized by sequence motifs involved in ATP binding and hydrolysis which are well conserved within each subgroup (see figure 5).

The complete biological role of HCV NS3 helicase is not yet known. It has been shown that it is essential for viral RNA replication and infectivity (Lindenbach et al. 2007). Furthermore, its role in virus particle assembly is assumed.

\subsection{NS3hel structure}

HCV NS3hel has three structural domains, two parallel $\alpha-\beta$ domains and one $\alpha$-helices-rich domain. These three domains are nearly equal in size and form a triangular shaped molecule. Between the two parallel $\alpha-\beta$ domains (RecA like domains 1 and 2 ) and domain 3 is a ssRNA binding groove. ssRNAs are bound through non-hydrophilic interactions between some of the residues and phosphate of the nucleic acid backbone. ATP binding is suggested between domain 1 and domain 2 (Kim, JL et al. 1998; Yao et al. 1997).

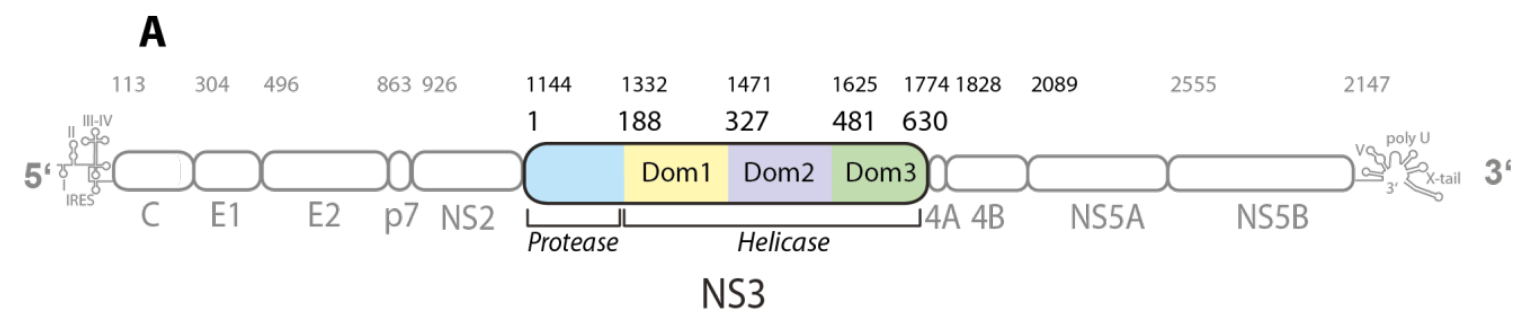

B

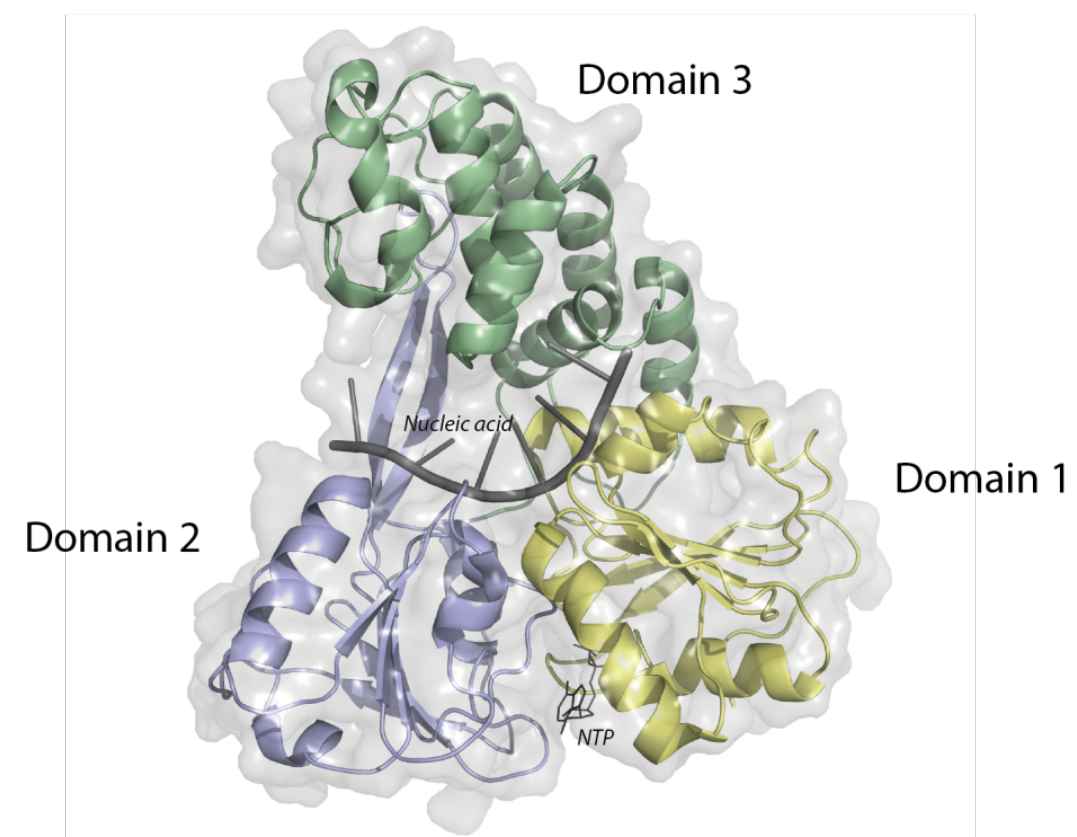

Figure 4 NS3hel domains. (A) Location of NS3hel in the genome of HCV. Full length NS3 is located from amino acid 1144 to 1774 in genotype 2a Jc1 sequence. Numbers highlight protein locations as well as locations of NS3hel domains. Numbering is based on full-length replicon Jc1. (B) Model of NS3hel in complex with single-stranded nucleic acid and NTP. Nucleic acids are bound in a binding groove between domain 1 and 2 and domain 3. NTPs are bound in a pocket between domain 1 and 2 (Gu and Rice 2010). NS3hel structure is based on Protein Data Bank Entry 3KQL and was rendered with PyMOL. 
As implied earlier NS3hel contains conserved helicase motifs associated with NTP binding, including Walker motif A and B (Motif I and II) (Walker et al. 1982). Walker motif A binds terminal phosphate groups of NTP cofactors. Walker motif B which includes signature sequence $\mathrm{DExH}$ functions as chelator of $\mathrm{Mg}^{2+}$ which is essential for NTP catalysis. Conserved helicase motifs line the groove between domain 1 and domain 2 and face the ssRNA binding site (Raney et al. 2010). The role of the highly conserved helicase motifs in unwinding nucleic acid duplexes was described in structure-function studies by different groups (Dillingham et al. 2001; Lin and Kim, JL 1999; Gu, M and Rice 2010).

A

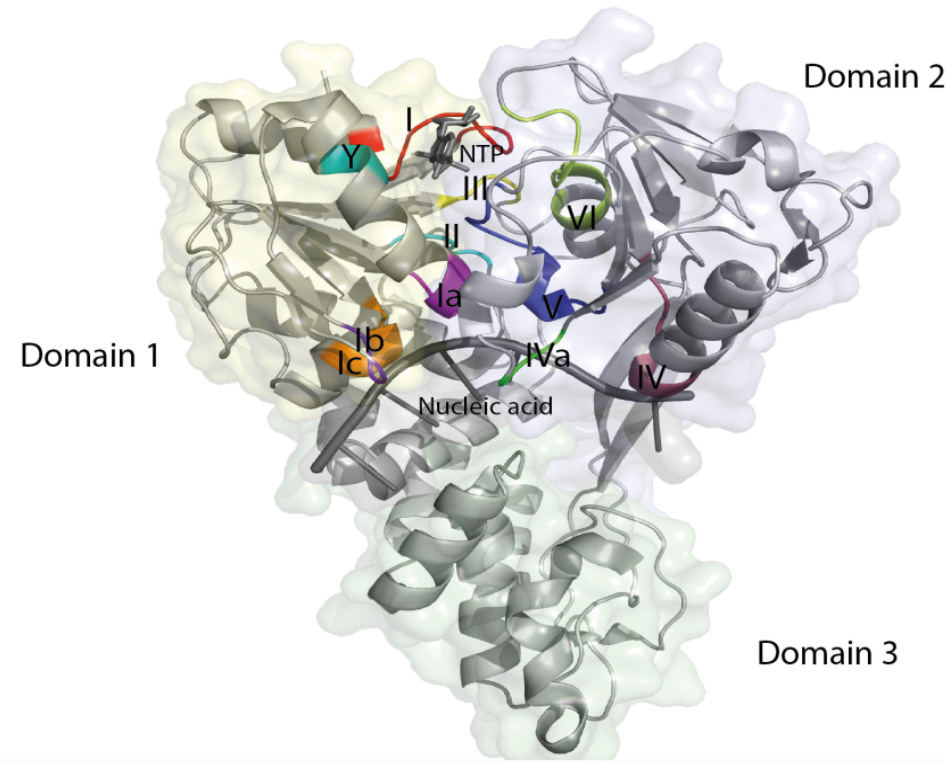

B

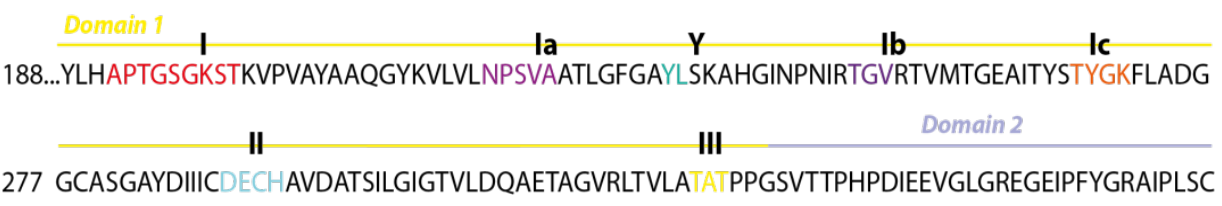

277 GCASGAYDIIICDECHAVDATSILGIGTVLDQAETAGVRLTVLATATPPGSVTTPHPDIEEVGLGREGEIPFYGRAIPLSC

$359 \underset{\text { IKGGRHLIFCHSKKKCDELAAALRGMGLNAVAYYRGLDVSIIPAQGDVVVVATDALMTGYTGDFDSVIDCNVAVTQ }}{\text { IV }}$

434 AVDFSLDPTFTITTQTVPQDAVSRSQRRGRTGRGRQGTYRYVSTGERASGMFDSVVLCECYDAGAAWYDLTPAETT

511 VRLRAYFNTPGLPVCQDHLEFWEAVFT GLTHIDAHFLSQTKQAGENFAYLVAYQATVCARAKAPPPSWDAMWKCL

538 ARLKPTLAGPTPLLYRLGPITNEVTLTHPGTKYIATCMQADLEVMT...630

Figure 5 Conserved helicase motifs of NS3. (A) Ribbon model of NS3. Conserved helicase motifs are colored and marked by Roman numbers. Structure is based on Protein Data Bank code 3KQL and rendered with PyMOL. (B) Primary structure of NS3hel. Motifs are colored as in A. Domains are highlighted with colored lines. Motifs are marked by Roman numbers. Domain 1 is colored yellow, domain 2 blue and domain 3 green matching to transparent surface colors of domains in A.

NS3hel binds RNA as well as DNA. The helicase unwinds duplex DNA and RNA in 3'-5' direction (Gu, M and Rice 2010). Both binding and unwinding of either RNA or DNA are pH 
dependent, they are enhanced at a $\mathrm{pH}$ around 6.5. This might suggest that HCV replication is located at an unique environment within the membranous web (Lam et al. 2004). Nucleic acids are bound more tightly to complete NS3 compared to NS3hel, indicating that the NS3 protease domain is involved in binding DNA or RNA (Raney et al. 2010).

\subsection{Mechanism of unwinding duplex DNA or RNA}

NTPases are bound in a pocket between domain 1 and domain 2. ATP is the main nucleotide for NTPase activity, but NS3 is non-selective hydrolyzing all ribonucleotides and desoxyribonucleotides (Bartenschlager et al. 2004). Motifs I, II, III and V play an important role in NTPase activity along with the newly described motif Y (NS3 residue Y241 in domain 1) (Gu, Rice 2010). Motif $Y$ is not found in other flavivirus helicases, which were supposed to be quite similar to HCV NS3hel. This residue is involved in sandwiching the nucleoside base with motif $\mathrm{V}$ residue T419. NS3hel can bind either dNTPs or NTPs as the helicase does not identify the 2' ribose oxygen (Suzich et al. 1993). Residues of motif I are indicated to stabilize the bound NTP for catalysis (Gu, M and Rice 2010).

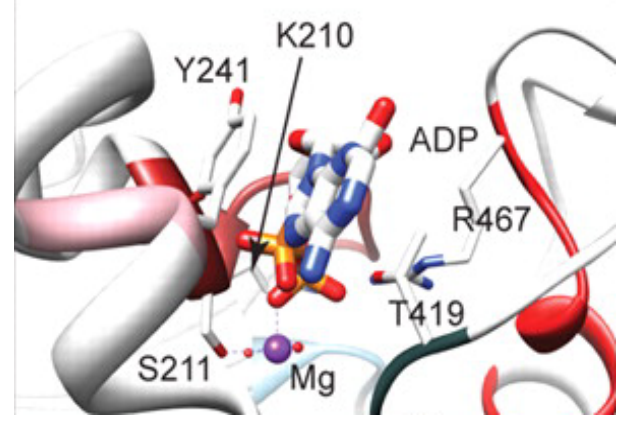

Figure 6 Important residues for binding NTPs. Figure modified from Raney et al. (2010) p. 22726. ATP transition state analog in the bound pocket between domain 1 and 2 is shown. Helicase motifs involved in NTPase activity are highlighted in colors. Motif I is colored red, motif $\mathrm{Y}$ is pink, motif III is light blue and motif $\mathrm{V}$ in dark green. Residues Y241 and T419 sandwich NTPs. This bound is stabilized by residues of motif $\mathrm{I}$.

The supposed inchworm model suggests translocation of the helicase by movement of domain 1 and 3 in the direction of domain 2 hydrolyzing ATP. Recently Gu and Rice (2010) made X-ray crystal structures of NS3 with ssDNA and ATP analogs to find out about the molecular mechanisms of unwinding and ATP hydrolysis of NS3hel. The group described three conformational stages of NS3hel using $\mathrm{ADP} \cdot \mathrm{BeF}_{3}$ and $\mathrm{ADP} \cdot \mathrm{AlF}_{4}^{-} \cdot \mathrm{ADP} \cdot \mathrm{BeF}_{3}$ mimics the ground state of ATP and ADP.AIF ${ }_{4}^{-}$the ATP hydrolysis state. These studies revealed a close view to functional mechanisms of the SF-2 helicase. Residues V432 (domain 2) and W501 (domain 3) are proposed to form a tight interaction with the nucleic acid. In absence of ATP five nucleotides of the nucleic acid are bound to NS3hel blocking unwinding function (Raney et al. 2010). Binding NTP leads to conformation change by rotation of domain 1 and 3 in the 
direction of domain 2 ( 5 ' direction) closing the RecA-like domains 1 and 2 . This reaction results in sliding of the nucleic acid of one nucleotide at the W501 position along the binding track in 5' direction. ATP hydrolysis leads to movement of domain 2 to its initial conformation. Within this change back to the initial state 1 base pair of duplex RNA or DNA gets unwinded. Residues V432 and W501 were shown to be important for unwinding by anchoring to the nucleic acid during conformation changes of the enzyme. Structure-based mutagenesis of V432 and W501 showed significant reduction of unwinding activity when mutating these residues (Lin and Kim, JL 1999). Essential for unwinding is a $\beta$-hairpin (with two conserved phenylalanine residues in the turn, also referred as Phe loop) within domain 2 (Saikrishnan et al. 2008). The $\beta$-hairpin is suggested to serve as a wedge splitting the double-stranded nucleic acid. Studies with a SF-1 helicase (RecD2) showed that a removal of the pin stops unwinding activity of the helicase demonstrating its important role in separating duplex DNA or RNA (Lam et al. 2003). 


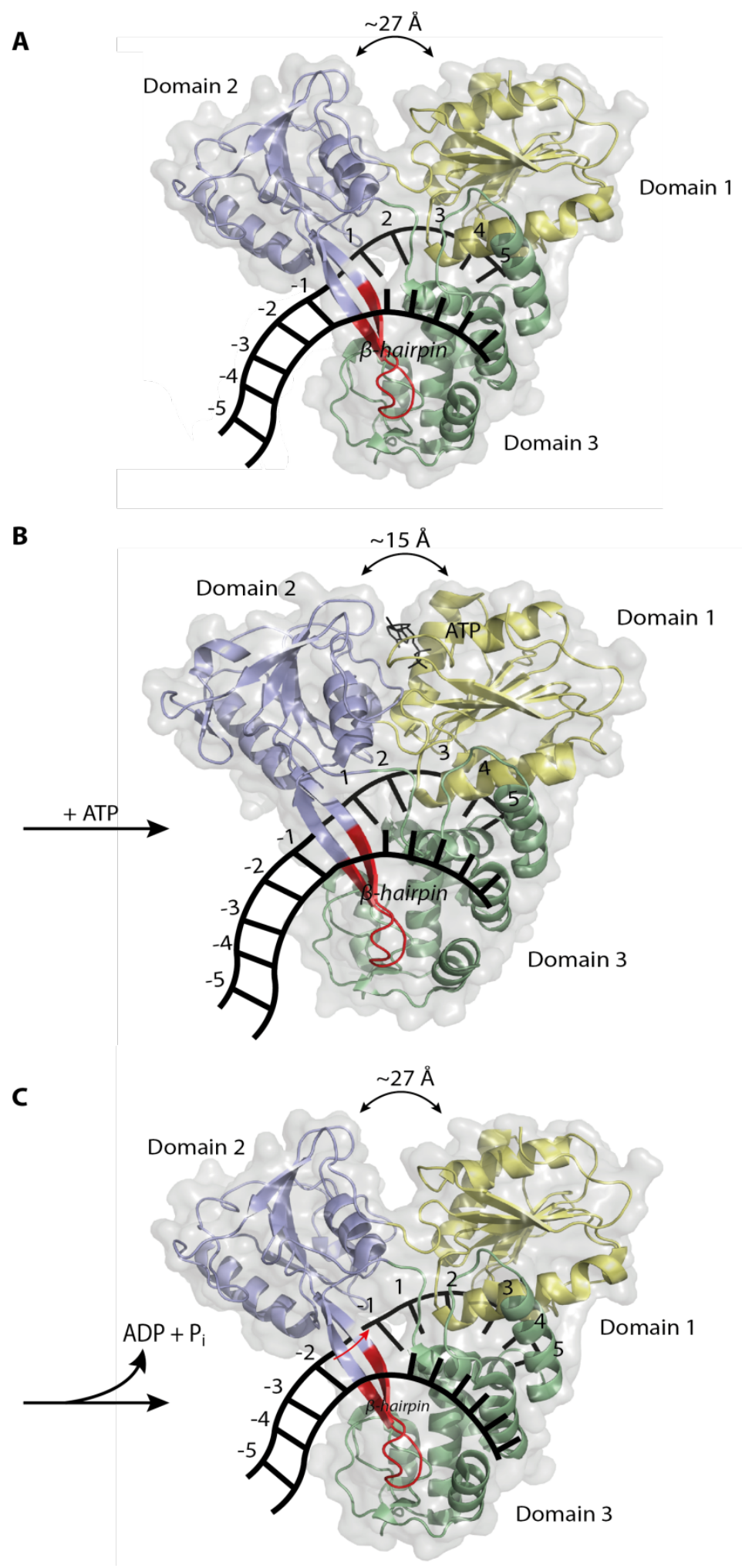

Figure 7 Model of NS3hel nucleic acid translocation and unwinding. Ribbon models of NS3 helicase domains are shown. Nucleic acids and ATP are simplified for clarity. Domains 1, 2 and 3 are colored yellow, blue and green. The $\beta$-hairpin structure is highlighted red. (A) Helicase domain with bound nucleic acid in the absence of NTP. Domain 1 and 2 are told to form a $27 \AA ̊$ gap (Gu, M and Rice 2010). (B) With binding ATP NS3hel undergoes a conformational change. Domains 1 and 3 are rotating in $5^{\prime}$ direction standing closer to domain 2 ( $\left.15 \AA\right)$ (Gu, M and Rice 2010). (C) ATP hydrolysis leads to rotation of domain 1 and 3 back to the initial conformation. These conformational changes lead to a movement of one nucleotide per hydrolyzed ATP which is indicated by the red arrow. The $\beta$-hairpin is described to function as wedge to split duplex DNA or RNA (Gu, M and Rice 2010). NS3hel structures are based on Protein data bank entry 3KQK and 3KQL. Models are rendered wit PyMOL. The figure was made with the help of the video clip from Gu, M and Rice (2010) (http://www.pnas.org/ content/suppl/2009/12/30/0913380107.DCSupplemental/SM1.mov). 


\subsubsection{NS4A}

As described above, NS4A is a cofactor of the NS3/4A serine protease. It is important for complete folding and positioning of the catalytic triad. Next to stabilizing the proteins conformation NS4A is told to serve as a membrane anchor with its N-terminal hydrophobic domain. Due to this function it is important for assembly of the replicase complex (Wölk et al. 2000). Furthermore, it is described to maintain multiple functions in the HCV life cycle. It is described to play a role in recognition of RNA by the NS3 protease and helicase. NS4A also interacts with NS4B, NS5A and uncleaved NS4B-5A (Lindenbach et al. 2007). The Cterminal region is suggested to influence NS5A hyperphosphorylation (Koch and Bartenschlager 1999).

\subsubsection{NS4B}

NS4B is a membrane-spanning protein with four central transmembrane domains. N- and Ctermini are facing the cytoplasm (Dubuisson 2007). It is suggested to play an essential role in inducing the formation of the membranous web which is important for organizing a membrane-bound replication complex (Lindenbach et al. 2007). Furthermore, NS4B reveals GTPase activity which is reported to be important for RNA replication (Einav et al. 2004). It is also described to inhibit RNA-dependent RNA polymerase activity of NS5B regulating HCV replication (Piccininni et al. 2002).

\subsubsection{NS5A}

NS5A is a phosphoprotein with an essential but unclear role in replication. NS5A contains three domains and an $\mathrm{N}$-terminal membrane anchor. Domain I includes a $\mathrm{Zn}^{2+}$-binding domain which is shown to be essential for replication (Tellinghuisen et al. 2005). NS5A is found in a phosphorylated (56 kDa) and a hyperphosphorylated (58 kDa) form. The role of the different phosphorylation states is not known. Hyperphosphorylation is modulated by NS4A. It is shown, that NS5A-hyperphosphorylation leads to enhancement of HCV RNA replication. These results indicate, that NS5A-hyperphosphorylation might down-regulate RNA replication by disrupting the interaction between NS5A and the human vesicleassociated membrane protein-associated protein A (hVAP-A) which is involved in intracellular vesicle trafficking and might be involved in the HCV replication machinery (Evans et al. 2004). NS5A is also suggested to modulate interferon (IFN) response. In this manner Domain II might play a role in INF-response by inhibiting protein kinase PKR suppressing a host cell IFN-induced antiviral pathway. 


\subsubsection{NS5B}

NS5B is a RNA-dependent RNA polymerase (RdRP). It is the major enzyme of viral RNA replication. It is, like most HCV proteins, post-translationally associated to the ER or ERderived membranes with its C-terminal hydrophobic tail of 21 aa (Ivashkina et al. 2002). NS5B shows structural similarities to other polymerases, a right hand structure with palm, finger and thumb domains (Lesburg et al. 1999). The palm domain represents the active site of the polymerase, fingers and thumb modulate RNA interactions. NS5B interacts with NS4B and NS5A (as described above) as well as NS3.

\subsection{HCV life cycle}

\subsubsection{Binding and cell entry}

The process of HCV entry is not yet understood. One can assume that multiple interaction between viral and target cell components lead to virus particle attachment and internalization. A number of possible receptors could be identified. CD81 from the tetraspanin family was shown to bind envelope glycoprotein E2 with its large extracellular loop (Pileri et al. 1998). However, CD81 is expressed in many different cell types which makes it unlikely that HCV entry into hepatocytes is dependent on this receptor only. Experiments using HCV pseudo particles (HCVpp) also revealed that HCV entry can be inhibited by antibodies against CD81 after attachment to target cells. It is suggested that CD81 functions as a coreceptor in a later step post-attachment (Cormier et al. 2004). Another potential receptor involved in HCV entry is the scavenger receptor class B type I (SR-BI). This receptor is highly expressed in hepatocytes mediating cholesterol uptake from lipoproteins. SR-BI also interacts with HCV E2 (Scarselli et al. 2002). Furthermore, E2 is reported to bind to C-type mannose-binding lectins DC-SIGN and L-SIGN as well as to the LDL-receptor (Cocquerel et al. 2006). Following attachment HCV entry is proposed to be internalized by receptormediated endocytosis (Lindenbach et al. 2007).

\subsubsection{RNA translation}

After HCV cell entry, viral RNA is release into the cytoplasm. The positive sense ssRNA serves as mRNA for protein synthesis. As described above HCV genome lacks a 5'-cap structure. Translation is initiated cap-independent by the IRES located in the 5'-NTR. IRES domain III binds directly to the $40 \mathrm{~S}$ ribosomal subunit and the initiating factor elF3. Thereby the AUG start codon, which is located within IRES at the beginning of the core region (nt 342 ), is placed directly on the $P$ position (peptidyl site) of the ribosome and translation is initiated without recruiting other initiation factors as elF4, elF4B etc (Tsukiyama-Kohara et al. 
1992). The complex structure of the IRES is important for correct location of the start codon to the 405 ribosomal subunit. It induces conformational changes within the ribosomal subunit closing the mRNA binding cleft which leads to an exact positioning of the AUG codon (Spahn et al. 2001). Hydrolysis of GTP and recruitment of the 605 subunit leads to an active translation complex. IRES-dependent translation can be inhibited by nt 428-442 of the core region by RNA-RNA interaction with nt 24-38 in domain I of the 5'NTR. This product inhibition might play a role in regulation of the virus life cycle, especially the rate of translation versus replication (Kim, YK et al. 2003).

\subsubsection{RNA replication}

HCV RNA replication depends on a membrane-associated replication complex. NS4B induces membrane alterations forming vesicles likely derived from the rough ER or intracellular lipid rafts (Bartenschlager et al. 2004). This so-called membranous web is indicated to form the replication complex as HCV proteins are shown to accumulate on these structures (Egger et al. 2002). NS3hel and NS5B RdRP most likely play the central role in HCV RNA replication as subgenomic replicons which only express NS3 and NS5B are able to replicate (Lohmann et al. 1999). NS3hel is able to unwind duplex RNA and DNA as well as RNA/DNA duplexes in 3' to 5' direction. Its role in replication is not yet understood. It might play a role in initiation of replication and elongation by unwinding duplexes. NS5B as RdRP is assumed to serve as the catalytic centre in HCV RNA replication (Bartenschlager et al. 2004). Positive-stranded RNA serves as a template of synthesis of negative-stranded RNA. Negative stranded RNA serves as template for positive strands RNA. It remains partially or fully double-stranded as replicative intermediates (RI) or replicative forms (RF) which serve as templates for semi-conservative, but asymmetrical RNA synthesis. Transcription leads to an almost ten-fold excess of positive-strands over negative-strands. Positive strands are used for translation or viral assembly (Bartenschlager et al. 2004).

\subsubsection{Virus assembly and release}

$\mathrm{HCV}$ particle assembly is not completely understood to date. It is likely that HCV particles integrate into ER membranes and get released over the secretory pathway. Recent studies hypothesized that HVC proteins E1, E2, p7, NS2, NS3 and NS5A are involved in virus particle assembly. Interactions between core and lipid droplets (LDs) were shown to be important as an initiating step in the assembly process (Boulant et al. 2007). A major role in assembly has been reported for NS5A. It is observed to co-localize with core on the surface of lipid droplets. Domain DI of NS5A was shown to interact with LDs which is crucial for virion production (Tellinghuisen et al. 2008). It is suggested that NS5A enables interactions 
between core and LDs building the scaffold for the assembly pathway. It is described to influence assembly by regulation of replication by uncoupling viral proteins from replication (Tellinghuisen et al. 2008). Furthermore, Stapleford and Lindenbach (2011) revealed that NS2 physically interacts with E1-E2 and NS3. It might play a central role in organizing a complex essential for virus particle assembly coordinating E1-E2 interaction with NS3. However, earlier studies assumed NS2 playing a role in a post-assembly step forming fully infectious viral particles (Jones, DM and McLauchlan 2010). lon channel p7 seems to be also involved in assembly acting within the assembly complex (Steinmann et al. 2007). NS3 was also described to be involved in particle assembly, but its role remains unclear.

\section{A}
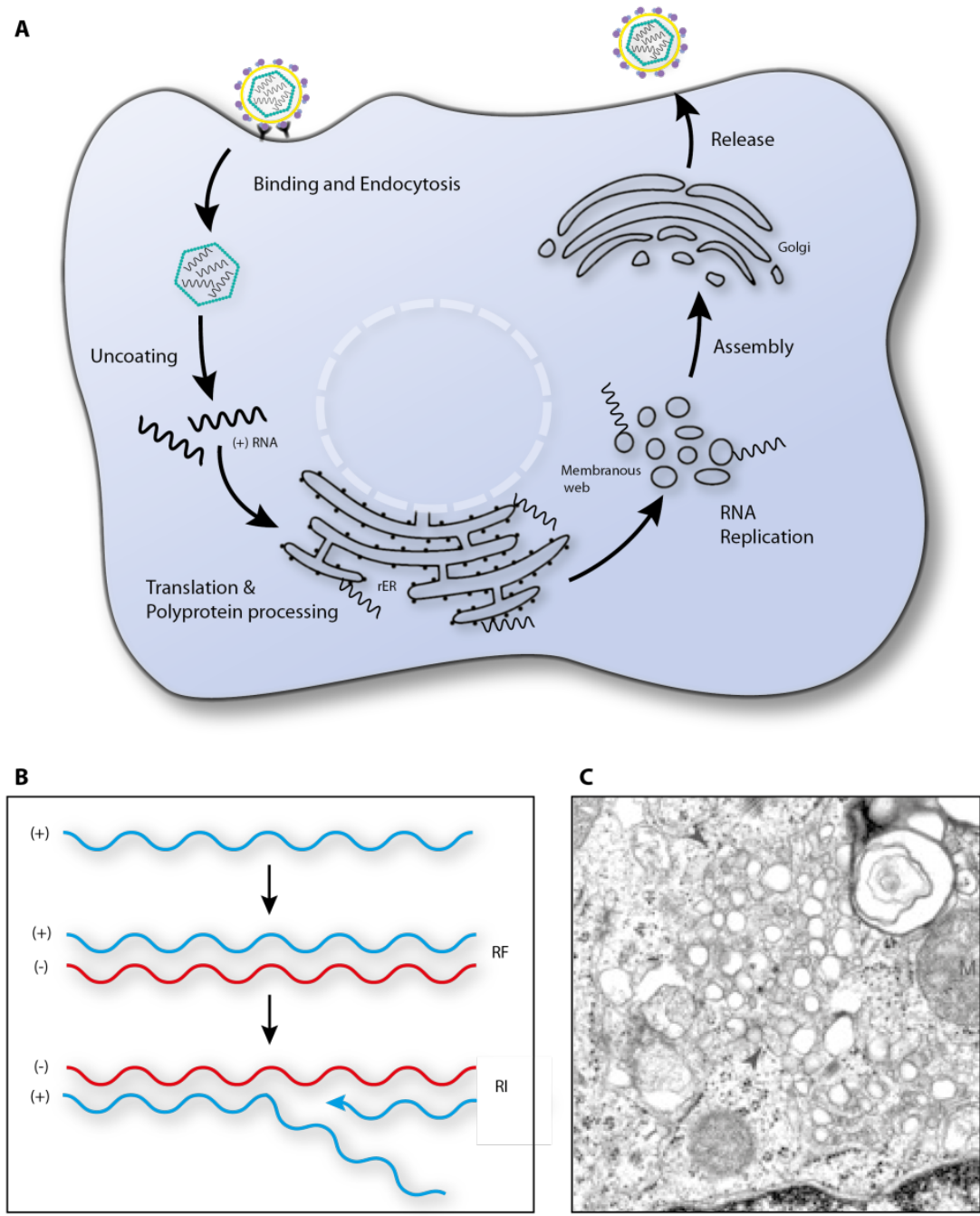

Figure $8 \mathrm{HCV}$ life cycle. (A) Model of life cycle. Constructed with the help of Lindenbach and Rice (2005) p. 934. (B) Mechanism of RNA replication. Positive stranded RNA serves as a template for negative strands. Negative strands remain partially or fully double stranded (RF or RI) and serve as templates for semi-conservative but asymmetrical RNA synthesis (Bartenschlager et al. 2004). (C) EM picture of the membranous web (Gosert et al. 2003). 


\subsubsection{HCV cell culture and replicon systems}

After its identification in the late 1980's advances in HCV research was held back by the lack of a cell culture model which allowed studying HCV infection in vitro. In 2000 the group of Charles Rice reported the first efficient cell culture system to study HCV replication. Adaptive mutations in NS5A and in the hepatoma cell line Huh 7 were required for HCV replication in vitro (Blight et al. 2000). These findings marked a major step in HCV research. Later on, in 2003, the group of Takaji Wakita discovered the genotype $2 a$ isolate $\mathrm{JFH}-1$ from a patient with fulminant hepatitis (Kato et al. 2003). The complete virus cell cycle can be studied with this isolate. It was shown that it is able to replicate, assemble and release infectious viral particle in Huh7 and Huh7.5 (a variation of Huh7 cells with more adaptive mutations) cells (Kato et al. 2003). These developments allowed new results in HCV research. But many questions are still unanswered, especially within the HCV life cycle and HCV infection. 


\subsection{Aim of this thesis}

The goal of the project was to determine NS3 helicase involvement in HCV virus particle assembly. It should be identified which regions in NS3hel contribute to virion assembly. Residues in all three NS3hel domains were screened by mutational analysis for their ability of releasing infectious virus particles. To identify determinants within NS3 that are important for virus assembly a structure-based alanine-scanning mutagenesis of NS3 was performed. As it is suggested that NS3 contributes to virus assembly through its interaction with NS2, surface residues were targeted. Well conserved sites were in the focus as virus assembly is an important step in the viral life cycle. As we just wanted to scan for problems in virus assembly, residues that are known to be essential for serine protease activity, helicase activity or viral genome replication were avoided. Because miss-folding of NS3 should also be avoided structural considerations, like surface charges and hydrogen bonds should not be changed as well. Altogether, surface residues in well conserved sites which are nonessential and structurally flexible were good candidates to be involved in particle assembly. 25 mutations within NS3hel that met these criteria were chosen to be scanned in this study. Furthermore, NS3hel sites essential for replication were also scanned. To examine if mutations of the chosen residues influence particle assembly, constructs were screened for replication and infectivity. Therefore passaging experiments with the HCV cell culture system were performed using full length Jc1 replicons and Huh 7.5 cells.

Constructs that were shown to replicate but not to produce infectious viral particles were of special interest for further studies. After confirming a defect in infectivity, these mutants were used to determine whether these residues are involved in viral assembly or release. Therefore, viral RNA within cell culture medium was monitored and intracellular particles were checked for infectivity.

It was also determined whether the identified residues are involved in interactions with NS2 playing a role in formation or stability of a newly described complex essential for virus particle assembly. Therefore, a protein-complex affinity purification system using streptavidin magnetic beads was used to capture NS2-NS3 interactions.

Altogether, in this study NS3 helicase domain was screened to identify regions essential for replication and infectivity. The involvement of NS3hel in particle assembly and its involvement in protein-protein interactions were further studied. 


\section{Material and Methods}

\subsection{Material}

\subsubsection{Cell lines and strains}

All cell lines were maintained in Dulbecco's modified Eagle medium (DMEM) (Invitrogen, Carlsbad, CA) supplemented with 10\% fetal calf serum (FCS) (HyClone, Logan, UT) and 1 $\mathrm{mM}$ nonessential amino acids (Invitrogen, Carlsbad, CA) at $37^{\circ} \mathrm{C}$ with $5 \% \mathrm{CO}_{2}$.

\subsubsection{Huh7.5 cells}

Huh7.5 cells are a highly permissive cell line of human hepatoma cells. This subline was developed from a Huh7 cell line harboring self-replicating subgenomic HCV replicons and being cured of HCV by prolonged treatment with INF- $\alpha$ by the Rice laboratory. Huh7.5 cells obtain a cellular environment that is highly permissive for HCV replication (Blight et al. 2002).

\subsubsection{Huh7.5/BirA cells}

Huh7.5/BirA cells expressed the E.coli biotin ligase BirA. Cells were generated by lentivirus introduction in the Lindenbach laboratory (Stapleford and Lindenbach 2011).

\subsubsection{E. coli}

$D H 5 \alpha^{\mathrm{TM}}$ is a well-known and versatile $E$. coli strain. The $D H 5 \alpha$ cell has mutations of $\varphi 80$ lacZ $\triangle \mathrm{M} 15$ and lacks the laqlq gene, which allows blue-white color screening of transformants with X-gal.

\begin{tabular}{lll}
\hline Strain & Genotype & Reference \\
\hline DH5 $\alpha$ & $\mathrm{F}^{\prime}(\Phi 80(\Delta / a c Z) \mathrm{M} 15) \Delta($ lacZYA-argF $)$ & www.catalog.takara- \\
& U169 recA1 endA1 hsdR17 rk- mk+ & bio.co \\
& supE44 thi-1 gyrA relA1 & \\
\hline
\end{tabular}

Table 2 Genotype of DH5 


\subsubsection{Growth media and buffer}

All growth media - liquid or solid - were prepared with twofold deionized water $\left(\mathrm{ddH}_{2} \mathrm{O}\right)$ and autoclaved for 30 minutes at $121^{\circ} \mathrm{C}$ before use. The right $\mathrm{pH}$-values were adjusted with $\mathrm{HCl}$ or $\mathrm{NaOH}$.

\subsubsection{Media for Escherichia coli cultures}

- $\quad$ LB media (pH 7.0)

$10 \mathrm{~g} \quad$ Bacto-Tryptone

$5 \mathrm{~g} \quad$ Bacto-Yeast Extract

$10 \mathrm{NaCl}$

$\mathrm{H}_{2} \mathrm{O} \quad$ to a final volume $1 \mathrm{~L}$

Supplementary for LB $_{\text {Amp }}-$ Media: $100 \mu \mathrm{g} / \mathrm{ml}$ Ampicillin

- $\quad$ LB Plates (pH 7.0)

$10 \mathrm{~g}$ Bacto-Tryptone

$5 \mathrm{~g} \quad$ Bacto-Yeast Extract

$10 \mathrm{~g} \mathrm{NaCl}$

15g Agar

$\mathrm{H}_{2} \mathrm{O}$ to a final volume $1 \mathrm{~L}$

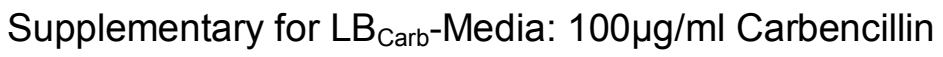

- $\quad$ SOB Medium (pH 7.0)

$20 \mathrm{~g}$ Bacto-Tryptone

$5 \mathrm{~g} \quad$ Bacto-Yeast Extract

$0.5 \mathrm{~g} \quad \mathrm{NaCl}$

$10 \mathrm{ml} 250 \mathrm{mM} \mathrm{KCl}$

$\mathrm{H}_{2} \mathrm{O}$ to a final volume $1 \mathrm{~L}$ 
2.1.2.2 Pulldown experiment \& Western blot buffer

\begin{tabular}{|c|c|}
\hline \multicolumn{2}{|c|}{ TBS } \\
\hline $20 \mathrm{mM}$ & Tris (pH 7.5) \\
\hline $150 \mathrm{mM}$ & $\mathrm{NaCl}$ \\
\hline \multicolumn{2}{|c|}{ TBS-Tx } \\
\hline $20 \mathrm{mM}$ & Tris, $\mathrm{pH} 7.5$ \\
\hline 150mM & $\mathrm{NaCl}$ \\
\hline$\%$ & Triton X-100 \\
\hline
\end{tabular}

Protein inhibitor

- $\quad$ Protein sample buffer

$50 \mathrm{mM} \quad$ Tris- $\mathrm{HCl}, \mathrm{pH} 6.8$

$2 \%$ sodium dodecylsulfate (SDS)

$0.1 \%$ bromophenol blue

$10 \%$ glycerol

$100 \mathrm{mM}$ DTT

- Transfer buffer $1 \mathrm{x}$

$25 \mathrm{mM}$ Tris base

190mM Glycine

$0.1 \%$ SDS

- 1x SDS-Page running buffer (pH 8.3)

25mM Tris

250mM Glycine

$0.1 \% \quad$ SDS 


$\begin{array}{ll}\text { - } & \text { PBS (pH 7.4) } \\ 80 \mathrm{~g} & \mathrm{NaCl} \\ 2 \mathrm{~g} & \mathrm{KCl} \\ 2 \mathrm{~g} & \mathrm{KH}_{2} \mathrm{PO}_{4} \\ 11.5 \mathrm{~g} & \mathrm{Na}_{2} \mathrm{HPO}_{4} \\ \mathrm{H}_{2} \mathrm{O} & \text { to final volume } 1 \mathrm{~L}\end{array}$

- PBS-T

$0.1 \%$ Tween-20

$1 \times$ PBS to final volume $1 \mathrm{~L}$

- $\quad 5 \%$ milk

$400 \mathrm{ml}$ PBS-T

$20 \mathrm{~g} \quad$ Non-fat dry milk

- 1x SDS sample buffer

50mM Tris HCl pH 6.8

$2 \% \quad$ SDS

$0.1 \% \quad$ Bromphenol blue

$10 \% \quad$ Glycerol

100mM DTT

\subsubsection{Antibodies}

\subsubsection{Primary Antibodies}

\begin{tabular}{llll}
\hline Primary Antibody & Raised in & Dilution & Source \\
\hline Anti-NS2 (clone 6H6) & Mouse & $1: 5000$ & Rice Laboratory, Rockefeller \\
& & & University, New York, NY \\
\hline Anti-NS3 (clone 9G2) & Mouse & $1: 5000$ & ViroGen Corporation, \\
& & & Watertown, MA \\
\hline Anti-NS5a (clone 9E10) & Mouse & $1: 20000$ & ViroGen Corporation, \\
& & & Watertown, MA \\
\hline
\end{tabular}

Table 3 Primary Antibodies 


\subsubsection{Secondary Antibodies}

\begin{tabular}{llll}
\hline Secondary Antibody & Raised in & Dilution & Source \\
\hline Anti-mouse HRP & Goat & $1: 5000$ & Jackson ImmunoResearch \\
& & & Laboratories, Inc, West \\
& & Groove, PA \\
\hline ImmPress anti-mouse & Goat & $1: 1$ & Jackson ImmunoResearch \\
HRP & & & Laboratories, Inc, West \\
& & & Groove, PA \\
\hline
\end{tabular}

Table 4 Secondary Antibodies

\subsubsection{Enzymes}

\begin{tabular}{ll}
\hline Enzyme & Source \\
\hline AfIII & New England Biolabs Inc, Ipswich, MA \\
\hline Bsp120I & Fermentas Inc, Glen Burnie, MD \\
\hline Clal & New England Biolabs Inc, Ipswich, MA \\
\hline Mung Bean Nuclease & New England Biolabs Inc, Ipswich, MA \\
\hline Proteinase K & Ambion, Austin, TX \\
\hline Superase-In & Ambion, Austin, TX \\
\hline T4 DNA-Ligase & New England Biolabs Inc, Ipswich, MA \\
\hline T4 DNA-Polymerase & New England Biolabs Inc, Ipswich, MA \\
\hline T7 RNA-Polymerase & Epicentre Biotechnologies, Madison, WI \\
\hline Xbal & New England Biolabs Inc, Ipswich, MA \\
\hline
\end{tabular}

Table 5 Used Enzymes

\subsubsection{Chemicals}

\begin{tabular}{ll}
\hline Chemicals & Source \\
\hline $1 \times$ DMEM & Invitrogen, Carlsbad, CA \\
\hline $1 \times$ DPBS & Invitrogen, Carlsbad, CA \\
\hline $30 \% \mathrm{H}_{2} \mathrm{O}_{2}$ & HACH Company, Loveland, CO \\
\hline
\end{tabular}




\begin{tabular}{|c|c|}
\hline $5.5 \mathrm{ml} 100 \times$ Non essential amino acids & Invitrogen, Carlsbad, CA \\
\hline Acrylamide (40\%) & American Bioanalytical, Inc., Natic, MA \\
\hline Agar & American Bioanalytical, Inc., Natic, MA \\
\hline Ammonium persulfat (APS) & American Bioanalytical, Inc., Natic, MA \\
\hline Bacto Tryptone & BD Biosciences, Franklin Lakes, NJ \\
\hline Bacto Yeast Extract & BD Biosciences, Franklin Lakes, NJ \\
\hline Bromphenol blue & BD Biosciences, Franklin Lakes, NJ \\
\hline Chloroform & American Bioanalytical, Inc., Natic, MA \\
\hline DMSO & Invitrogen, Carlsbad, CA \\
\hline DTT & American Bioanalytical, Inc., Natic, MA \\
\hline Ethanol & American Bioanalytical, Inc., Natic, MA \\
\hline Ethidium Bromide & Sigma-Aldrich Corp., St. Louis, MO \\
\hline Ethylenediaminetetraacetic acid (EDTA) & American Bioanalytical, Inc., Natic, MA \\
\hline Fetal Calf Serum (lot\# frf26747) & HyClone, Logan, UT \\
\hline Glycerol & American Bioanalytical, Inc., Natic, MA \\
\hline Potassium chloride & Baker Bioscience, Sanford, ME \\
\hline $\mathrm{KH}_{2} \mathrm{PO}_{4}$ & Baker Bioscience, Sanford, ME \\
\hline L.M.P. Agarose & Promega, Fitchburg, WI \\
\hline LE Agarose & Promega, Fitchburg, WI \\
\hline Magnesium chloride & J.T. Baker, Phillipsburg, NJ \\
\hline Methanol & American Bioanalytical, Inc., Natic, MA \\
\hline $\mathrm{Na}_{2} \mathrm{HPO}_{4}$ & American Bioanalytical, Inc., Natic, MA \\
\hline Non-fat dry milk & American Bioanalytical, Inc., Natic, MA \\
\hline Phenol & Roche, Basel, Switzerland \\
\hline Ponceau S & DiaSys Ltd, Berkshire, UK \\
\hline Renilla $5 x$ lysis buffer & Promega, Fitchburg, WI \\
\hline Sodium acetate & Baker Bioscience, Sanford, ME \\
\hline Sodium Cloride & J.T. Baker, Phillipsburg, NJ \\
\hline Tetramethylethylendiamine (TEMED) & Promega, Fitchburg, WI \\
\hline Tris base & Roche, Basel, Switzerland \\
\hline Tris buffer & QUIAGEN, Valencia, CA \\
\hline
\end{tabular}




\begin{tabular}{ll}
\hline Triton X-100 & Calbiochem, Merck, Darmstadt, Germany \\
\hline Trypsine EDTA 0,05\% & Invitrogen, Carlsbad, CA \\
\hline Tween-20 & Sigma-Aldrich Corp., St. Louis, MO \\
\hline Dynabeads ${ }^{\text {TM }}$ M-280 & Invitrogen, Carlsbad, CA \\
\hline
\end{tabular}

\subsubsection{Kits}

\begin{tabular}{ll}
\hline Kit & Source \\
\hline Gaussia Luciferase Kit & New England Biolabs Inc, Ipswich, MA \\
\hline HiSpeed Plasmid Midi Kit & QUIAGEN, Valencia, CA \\
\hline Immpact DAB & Vector Labs, Burlingame, CA \\
\hline LightCycler RNA Amplification Kit & Roche, Basel, Switzerland \\
\hline QIAprep Spin Miniprep Kit & QUIAGEN, Valencia, CA \\
\hline QUI quick PCR purification Kit & QUIAGEN, Valencia, CA \\
\hline RNAeasy Mini Kit & QUIAGEN, Valencia, CA \\
\hline SuperSignal West Pico Western Blotting & Thermo Scientific, Rockford, IL \\
Kit & \\
\hline
\end{tabular}

Table 7 Used Kits

\subsubsection{Equipment}

\begin{tabular}{ll}
\hline Equipment & Source \\
\hline $15 \mathrm{~cm}$ Cell culture dishes & BD Biosciences, Franklin Lakes, NJ \\
\hline 6 Wells Cell culture plates & BD Biosciences, Franklin Lakes, NJ \\
\hline 96 Wells Cell culture plates & BD Biosciences, Franklin Lakes, NJ \\
\hline Air Clean 600 PCR Workstation & AirClean ${ }^{\circ}$ Systems, Raleigh, NC \\
\hline Analytic balance & METTLER TOLEDO, Columbus, OH \\
\hline Balance & METTLER TOLEDO, Columbus, OH \\
\hline $70 \mu l$ nylon cell strainer & BD Biosciences, Franklin Lakes, NJ \\
\hline Centrifudge & Beckman Coulter, Brea, CA \\
\hline Centrifuge 5415D & Eppendorf, Hamburg, Germany \\
\hline
\end{tabular}




\begin{tabular}{|c|c|}
\hline Centrifuge 5415R & Eppendorf, Hamburg, Germany \\
\hline Centro LB 960 & Berthold, Oak Ridge, TN \\
\hline Digital Camera & Sony, Tokyo, Japan \\
\hline Incubator $37^{\circ} \mathrm{C}$ & Thermo Scientific, Rockford, IL \\
\hline LightCycler 480 & Roche, Penzberg, Germany \\
\hline Nano Drop Spectrophotometer ND-100 & Thermo Scientific; Delaware, USA \\
\hline Power Pac HV & BioRad, Hercules, CA \\
\hline Tetrad 2 ThermalCycler & BioRad, Hercules, CA \\
\hline Thermomixer $\mathrm{R}$ & Eppendorf, Hamburg, Germany \\
\hline UV-Illuminator & Stratagene, Santa Clara, CA \\
\hline Long wave UV-box Bi-O-Vision & Spectroline, Westbury, NY \\
\hline Vortex Mixer & Fisher Scientific, Waltham, MA \\
\hline Iso Temp 110 Waterbath & Fisher Scientific, Waltham, MA \\
\hline Allegra $x-15 R$ Centrifuge & Beckman Coulter, Brea, CA \\
\hline Nikon Eclipse TS100 inverted microscope & Nikon, Tokyo, Japan \\
\hline 96 well plate qRT-PCR & Roche, Penzberg, Germany \\
\hline Nutator & Artisan Scientific, Champaign, IL \\
\hline MagnaRack ${ }^{\mathrm{TM}}$ & Invitrogen, Carlsbad, CA \\
\hline Bio Trace PVDF Membrane & $\begin{array}{l}\text { Pall Life Sciences, Port Washington, } \\
\text { NY }\end{array}$ \\
\hline ECM 830 Electro Square Porator BTX & Harvard Apparatus, Holliston, MA \\
\hline SterilGARD III Advance Hood & The Baker Company, Sanford, ME \\
\hline $\begin{array}{l}\text { Forma Series II Water-Jacketed CO2 Incubator } \\
\text { HEPA Class } 100\end{array}$ & Thermo Scientific, Rockford, IL \\
\hline
\end{tabular}




\subsubsection{Software}

\begin{tabular}{ll}
\hline Software & Source \\
\hline Adobe Design Premium CS5 & Adobe Systems \\
\hline ApE & M. Wayne Davis \\
\hline DNA Strider & Yizhou Joseph He \\
\hline Microsoft Office 2007 & Mircrosoft Corporation \\
\hline PyMOL & DeLano Scientific LLC, Schrödinger \\
\hline SeqMan & DNASTAR, Inc. \\
\hline
\end{tabular}

Table 9 Used Software 


\subsection{Methods}

\subsubsection{Cloning}

The Jc1 chimera is a construct made by inserting NS2 codons $30-217$ of JFH1 into the pJ6/JFH1 chimeric genotype 2a HCV cDNA clone (Lindenbach Lab described in Phan et al. 2009). pJ6/JFH1 is a hybrid of the core to NS2 region of J6 and the subgenomic replicon JFH1 (Jones, CT et al. 2007). Jc1 is capable of yielding 100-1000-fold higher yields than any of the other HCV replicons (Pietschmann et al. 2006). Figure 9 gives an overview of all used Jc1 constructs.

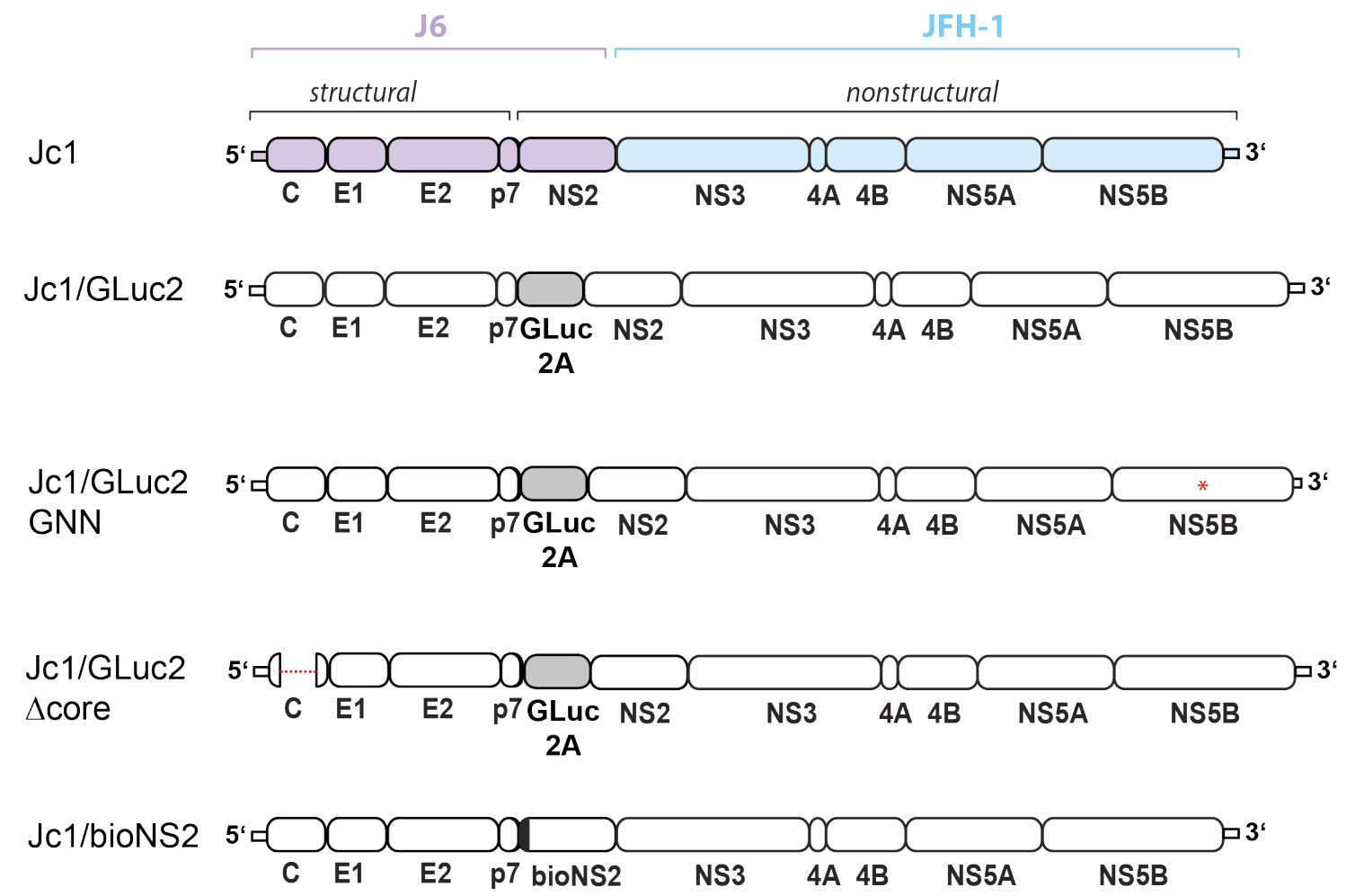

Figure $9 \mathrm{Jc1}$ constructs. All used constructs are compared. All constructs are based on the full length replicon Jc1 which is a hybrid of J6 and JFH1. The part of J6 origin is marked purple and the JFH-1 part is marked blued. Position of the GLuc2A gene is shown highlighted grey. Mutations within GNN are marked by the red star and $\Delta$ core deletions are marked by red dots. Insertion of the biotin acceptor peptide is also shown; site of insertion is marked in black.

\subsubsection{Jc1/GLuc2 clones}

The used vector plasmid Jc1/GLuc2 was constructed by the Lindenbach laboratory. GLuc2A reporter gene was constructed by inserting a silent Mlul site between p7 and NS2 by site directed mutagenesis. This site was used to insert the Gaussia princeps luciferase reporter gene and 21 codons of the foot-and-mouth-disease-virus (FMDV) 2A 'autocleaving' gene. 
200-500ng plasmid-DNA (constructs made by Rudolph K. Béran by site-directed mutagenesis; named pJRB) were digested with EcoRI-HF and BsrGI. Jc1 as vector was also digested with EcoRI and. Jc1 clones were named JGN3.
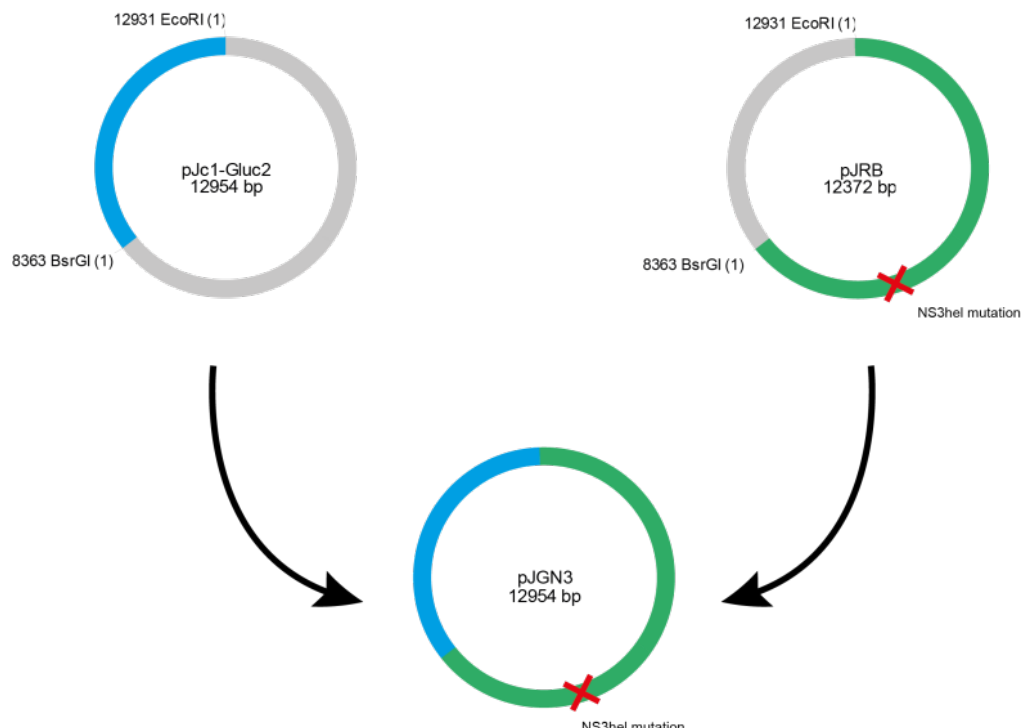

Figure 10 Cloning of pJGN3. Plasmids JC1 and JRB were digested with restriction enzymes in order to clone the NS3hel mutation into the full-length replicon Jc1. Enzymes used were EcoRI and BsrGI. Restriction sites and their positions are shown. Numbers of an enzyme's total restriction sites within each plasmid are shown in brackets. Positions are counted according to the full-length replicon Jc1. Figure made with ApE after the respective plasmid's sequence.

Reaction components were assembled as follows:

$\begin{array}{ll}200-500 n g & \text { DNA } \\ 1 \mu \mathrm{l} & \text { NEB buffer 4 (10x) } \\ 0.1 \mu \mathrm{l} & \text { BSA (100x) } \\ 0.2 \mu \mathrm{l} & \text { AfIII } \\ 0.2 \mu \mathrm{l} & \text { BsrGI } \\ 0.2 \mu \mathrm{l} & \text { Clal } \\ \text { to } 10 \mu \mathrm{l} & \mathrm{H}_{2} \mathrm{O}\end{array}$

\subsubsection{Jc1/bioNS2 clones}

Jc1/bioNS2 constructs were made by the Lindenbach laboratory by insertion of the biotin acceptor peptide (BAP). Oligos YO-315 and YO-316 were introduced into the Mlul site (described above) of Jc1. 


\begin{tabular}{ll}
\hline Primary structure of BAP & GLNDIFEAQKIEWHE \\
\hline Sequence of YO-315 & 5'CGCGCCGGGCCTGAACGACATCTTCGAG \\
& GCCCAGAAGATCGAGTGGCACGGCGGCA \\
& GCGGCGGCAGCGA-3' \\
\hline Sequence of YO-316 & 5'CGCGTCGCTGCCGCCGCTGCCGCCGTGC \\
& CACTCGATCTTCTGGGCCTCGAAGATGTCG \\
& TTCAGGCCCGG-3' \\
\hline
\end{tabular}

Table 10 Sequence of BAP and Oligos YO-315 and YO-316

200-500ng of JGN3 plasmid DNA and the Jc1/bioNS2 vector were digested with EcoRI and Mlul.
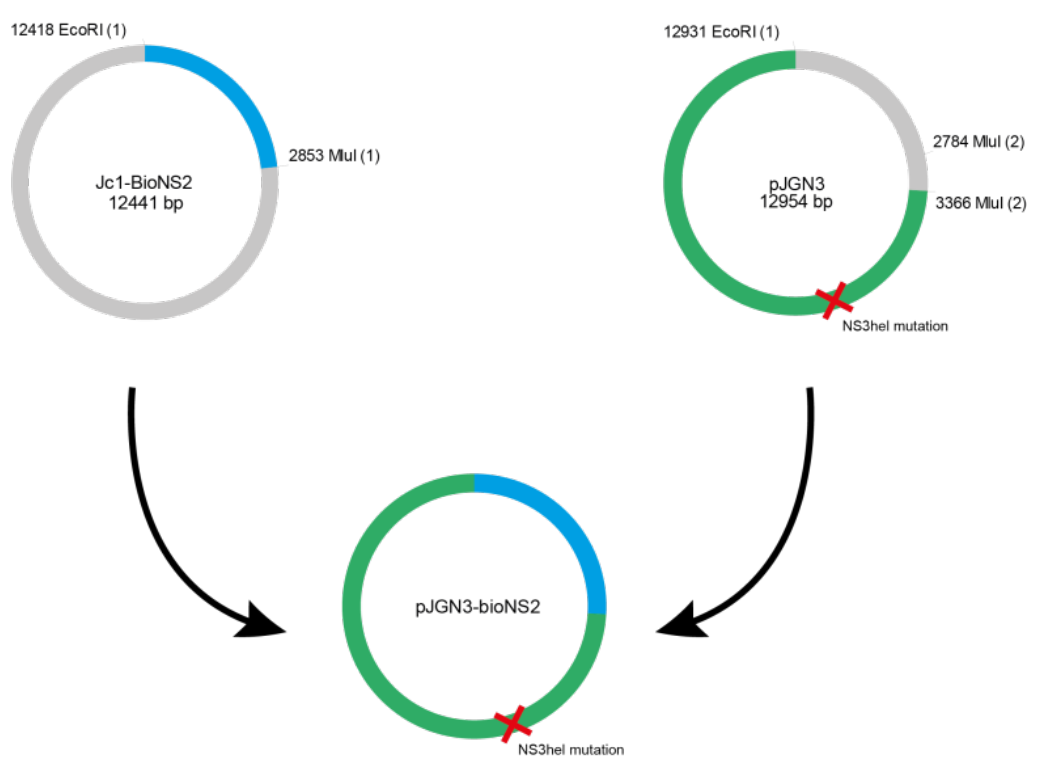

Figure 11 Cloning of Jc1/BioNS2 plasmids. Plasmids Jc1-BioNS2 and JGN3 were digested with restriction enzymes in order to clone the NS3hel mutation into the biotin acceptor peptide (BAP) tagged full length replicon Jc1-BioNS2. Restriction sites and their positions are shown. Number of an enzyme's total restriction sites within each plasmid are shown in brackets. Positions are counted according to the full-length replicon Jc1. Figure made with ApE after the respective plasmid's sequence.

Reaction components were assembled as follows:

$\begin{array}{ll}200-500 n g & \text { DNA } \\ 1 \mu \mathrm{l} & \text { NEB buffer 3 (10x) } \\ 0.1 \mu \mathrm{l} & \text { BSA (100x) } \\ 0.2 \mu \mathrm{l} & \text { EcoRI-HF } \\ 0.2 \mu \mathrm{l} & \text { Mlul } \\ \text { to } 10 \mu \mathrm{l} & \mathrm{H}_{2} \mathrm{O}\end{array}$


Reactions were incubated at $37^{\circ} \mathrm{C}$ for 4 hours.

Fragments were separated by electrophoresis in a thin glass-slide gel, in $0.8 \%$. Low melting point agarose in $1 \times$ TAE buffer. The gel was stained in ethidium bromide $(\mathrm{EtBr})$. Bands were cut out on a long-wave UV box and $50 \mu \mathrm{L} \mathrm{T}_{10} \mathrm{E}_{0.1}$ and $1 \mu \mathrm{l} 1 \mathrm{M} \mathrm{MgCl}_{2}$ were added to each fragment. Fragments were melted for 2 to 5 minutes at $72^{\circ} \mathrm{C}$.

For ligation of the fragments with the vector fragment reactions were assembled as follows:

$\begin{array}{ll}2 \mu \mathrm{l} & \text { Vector } \\ 10 \mu \mathrm{l} & \text { Insert } \\ \text { to } 17 \mu \mathrm{l} & \mathrm{H}_{2} \mathrm{O} \\ 2 \mu \mathrm{l} & 10 \mathrm{x} \text { ligation buffer (New England Biolabs }{ }^{\circledR} \text { ) } \\ 1 \mu \mathrm{l} & \text { T4 DNA ligase }\end{array}$

Ligations were done at room temperature anywhere from 10 minutes to a few hours or at $16^{\circ} \mathrm{C}$ overnight.

Ligations were briefly melted at $72^{\circ} \mathrm{C}$ and $10 \mu \mathrm{l}$ were used to transform competent bacteria.

\subsubsection{Plasmid transformation into E.coli}

The used cloning strain was DH5a. The plasmids Jc1 and JGN3 were introduced into an aliquot of $100 \mu \mathrm{l}$ competent cells by heat shock transformation. Therefore $10 \mu \mathrm{l}$ of the plasmid was added to the aliquot and incubated on ice for 30 minutes. Then the cells were heat shocked in a $42^{\circ} \mathrm{C}$ water bath for 45 seconds and cooled down on ice for 2 minutes. After adding $900 \mu \mathrm{L}$ SOB media to the $\mathrm{DH} 5 \alpha /$ plasmid mix the cells were incubated at $37^{\circ} \mathrm{C}$ for 1 hour while shaking. Afterwards the cells were pated on $\mathrm{LB}_{\text {carb }}$ plates. The plates were incubated at $37^{\circ} \mathrm{C}$ over night.

\subsubsection{Purification of Plasmid-DNA}

Transformed cells were picked from the $\mathrm{LB}_{\text {carb }}$ plates and incubated in $50 \mathrm{ml}$ liquid $L B_{\text {amp }}$ over night at $37^{\circ} \mathrm{C}$.

For confirmation of the right plasmids 'QIAprep Spin Miniprep Kit' was used. Isolated and purified plasmid-DNA was used for enzymatic digestion and run on a $0.8 \%$ agarose gel.

For isolation and purification of E.coli plasmid DNA the 'HiSpeed Plasmid Midi Kit' was used. Purified plasmid-DNA was used for sequencing and further experiments. 


\subsubsection{Sequencing}

All constructs were verified by DNA sequencing at the W.M. Keck Foundation Biotechnology Resource Center at Yale University following the facilities' instructions.

\begin{tabular}{|c|c|c|c|}
\hline Name & Region & Orientation & Sequence \\
\hline YO-0363 & NS3 & Reverse & 5'-TCGACGGCTCCACACTTGCAC-3' \\
\hline YO-0364 & NS3 & Forward & 5'-TGCTGAGGGGGACTTGGTAG-3' \\
\hline YO-0365 & NS3 & Reverse & 5'-GCCGTAGCCAGCACAGTTAGTCTG-3’ \\
\hline YO-0366 & NS3 & Forward & 5'-CGATGAATGCCACGCTGTGGATGCT-3' \\
\hline YO-0367 & NS3 & Reverse & 5'-GGGAGAGGAAGTGGGCGTCTA-3' \\
\hline YO-0368 & NS3 & Forward & 5'-CCGTCAGGCTTAGAGCGTATTTCA-3' \\
\hline YO-0369 & NS3 & Reverse & 5'-AGTGTTGACAATCCTGCGAGGTATT-3’ \\
\hline YO-0073 & bioNS2 & Reverse & 5'-TCCTTGCACACCGGCTTTA-3' \\
\hline YO-0074 & bioNS2 & Forward & 5'-GCCGCATTTGAGGTAAGTGGTA-3' \\
\hline YO-0075 & bioNS2 & Reverse & 5'-TTCAACGCCAGCACGGACCTGT-3’ \\
\hline YO-0076 & bioNS2 & Forward & 5'-TCCATAAGCAGGCGCAAAC-3' \\
\hline
\end{tabular}

Table 11 Primers used for Sequencing

\subsubsection{In vitro transcription}

$20 \mu \mathrm{g}$ plasmid-DNA was used to linearize with Xbal to prepare transcription templates.

The reaction components were assembled as follows:

$\begin{array}{ll}20 \mu \mathrm{g} & \text { DNA } \\ 10 \mu \mathrm{l} & \text { NEB buffer } 4(10 \mathrm{x}) \\ 1 \mu \mathrm{l} & \text { BSA }(100 \mathrm{x}) \\ 3 \mu \mathrm{l} & \text { Xbal } \\ \text { to } 100 \mu \mathrm{l} & \mathrm{H}_{2} \mathrm{O}\end{array}$

The digestion was done over night in a $37^{\circ} \mathrm{C}$ water bath.

5 ' overhangs were chewed back with Mung bean nuclease. Therefore $2 \mu \mathrm{l}$ Mung bean nuclease was added to the reaction and incubated at room temperature for 15 minutes.

The reaction was cleaned up by adding $5 \mu \mathrm{l} 10 \%$ SDS and $3 \mu \mathrm{l}$ Proteinase $\mathrm{K}(18 \mathrm{mg} / \mathrm{ml})$ and incubated at $50^{\circ} \mathrm{C}$ for 30 minutes. 
Afterwards a Phenol/Chloroform Extraction was performed. Phenol/Chloroform $\left(\mathrm{CHCl}_{3}\right)(1: 1)$ was prepared by using Tris-buffered phenol. Phenol/ $\mathrm{CHCl}_{3}$ was pre-warmed 15 to 30 minutes before use.

The linearized and cleaned DNA was mixed 1:1 with phenol/ $\mathrm{CHCl}_{3}$ and then vortexed and spined for 5 minutes at maximum speed. The top layer was removed and the extraction repeated once.

The Phenol/Chloroform Extraction was followed by ethanol precipitation. Therefore 1/10 volume RNA-grade 3M NaOAc ( $\mathrm{pH} 5.2$ ) and 3 volumes $100 \%$ ethanol (EtOH) were added. DNA was pelleted by spinning 10 minutes at $4^{\circ} \mathrm{C}$ at top speed. The supernatant was removed and the pellet was washed with $1 \mathrm{ml} 70 \% \mathrm{EtOH}$. DNA was resuspended in $20 \mu \mathrm{l}$ RNA-grade $\mathrm{T}_{10} \mathrm{E}_{0.1}$.

For the in vitro transcription the master mix was assembled as follows:

$\begin{array}{ll}4 \mu \mathrm{l} & 5 x \text { transcription buffer (Epicentre) } \\ 2 \mu \mathrm{l} & 100 \mathrm{mM} \text { DTT } \\ 3.8 \mu \mathrm{l} & \mathrm{H}_{2} \mathrm{O} \\ 4.8 \mu \mathrm{l} & 25 \mathrm{mM} \mathrm{MgCl}_{2} \text { (Roche) } \\ 2.4 \mu \mathrm{l} & \text { NTP mix (25mM of each NTP) } \\ 0.4 \mu \mathrm{l} & \text { T7 RNAP (Epicentre), 50 U/ } \mu \mathrm{l} \\ 0.6 \mu \mathrm{l} & \text { SUPERasin (Ambion) }\end{array}$

$2 \mu \mathrm{l}$ of template was added last and incubated at $37^{\circ} \mathrm{C}$ for 1.5 hours.

The reaction was cleaned up with the 'RNAeasy mini kit'. The volume was adjusted to get $100 \mu \mathrm{RNA} / \mu \mathrm{l}$ and $10 \mu \mathrm{l}$ were used for transfections.

\subsubsection{Cell culture methods}

\subsubsection{General conditions}

All cell culture work was performed in a biosafety level 2+ cabinet licensed by the State of Connecticut Department of Public Health. All cells were grown in cell culture dishes (BD Biosciences) in a $5 \% \mathrm{CO}_{2}$ air incubator at $37^{\circ} \mathrm{C}$.

Huh 7.5 cells (human hepatoma cell line) were maintained in Dulbecco's Modified Eagle's Medium (DMEM) containing high glucose, sodium pyruvate and L-glutamine, $10 \%$ heat inactivated fetal calf serum (FCS) and $1 \%(100 \mu \mathrm{M})$ nonessential amino acids. Cells were typically split $1: 3$ every three days. They were maintained in a sub-confluent state $(\sim 80 \%$ 
confluent) to avoid overgrowing, which would lead to continuous clumping and decreased efficiency.

A

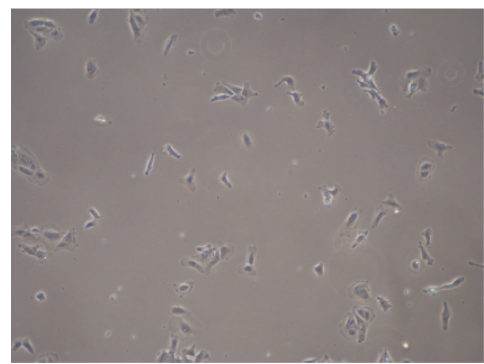

B

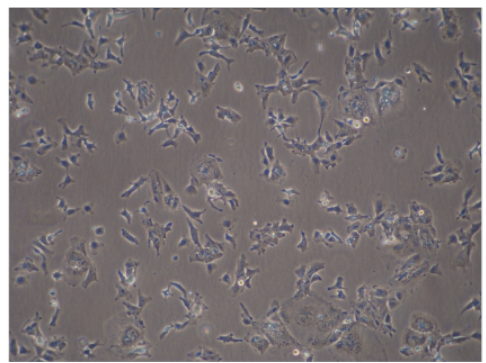

C

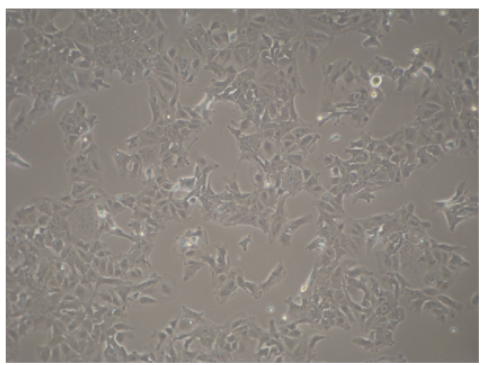

Figure 12 Huh 7.5 cells in different stages of growth after splitting. (A) Cells $1 \mathrm{~d}$ after they were split. (B) $2 \mathrm{~d}$ post splitting. Cells were around $40-60 \%$ confluent. (C) $3 \mathrm{~d}$ after splitting. Cells were around $80 \%$ confluent. They were ready to split.

To split cells media was aspirated and the cells were washed with Dulbecco's PBS. $3 \mathrm{ml}$ trypsin per $15 \mathrm{~cm}$ dish (P150) was added and incubated at $37^{\circ} \mathrm{C}$ for 5 minutes. An equal volume of media was added and cells were gently resuspended by pipetting, pooled and placed in a $50 \mathrm{ml}$ Falcon tube using a $70 \mu \mathrm{m}$ nylon cell strainer to maintain the best possible separation of the cells and avoid cell clumping. Cells were plated on new $15 \mathrm{~cm}$ plates $1: 3$ in $20 \mathrm{ml}$ media.

BirA expressing Huh7.5 cells were made by lentivirus transduction by the Lindenbach Laboratory.

\subsubsection{Transfections}

Cells were transfected when around $80 \%$ confluent. Plates were washed and trypsinized as if they would normally be split (see 2.2.6.1) and collected in $50 \mathrm{ml}$ tubes. The cells for the transfection were centrifuged at $1200 \mathrm{rpm}$ at $4^{\circ} \mathrm{C}$ for 6 minutes. Then the media was aspirated without disturbing the pellet. $5 \mathrm{ml}$ transfection PBS $\left(\sim 4^{\circ} \mathrm{C}\right)$ was added to re-suspend the pellet and another $35 \mathrm{ml}$ transfection PBS was added afterwards. The cells were centrifuged again at $1200 \mathrm{rpm}$ at $4^{\circ} \mathrm{C}$ for 6 minutes. Before the centrifugation $10 \mu \mathrm{l}$ of the cell suspension were removed for cell count. Therefore an equal volume of tryptan blue (dilution factor 2) to stain dead cells was added to the cell suspension. A hemocytometer was used to count the average cell count per quadrant under the light microscope. The hematocytometer volume of each quadrant is $0.1 \mu \mathrm{l}$. The total number of cells was determined with the following equation:

(average cell count per quadrant $) \times\left(1 \times 10^{4}\right) \times 2 \times($ ml of cells $)=$ total number of cells 
After the second centrifugation PBS was aspirated, the cells were re-suspended in $5 \mathrm{ml}$ transfection PBS and then $35 \mathrm{ml}$ of PBS was added as described. After another centrifugation at $1200 \mathrm{rpm}$ at $4^{\circ} \mathrm{C}$ PBS was aspirated again and the cells were re-suspended to a volume needed to yield $2 \times 10^{7}$ cells $/ \mathrm{ml}$ :

$$
\frac{\text { total number of cells }}{2 \times 10^{7}}=m l P B S \text { to add to get a yield of } 2 \times 10^{7}
$$

$380 \mu \mathrm{l}$ cells with the appropriate yield were then electroporated with $1 \mu \mathrm{g}$ replicon RNA. For the electroporation a square-wave electroporator set with 5 pulses at $820 \mathrm{~V}$ for $99 \mu \mathrm{sec}$ with a pause of 1.1 seconds between each pulse was used.

After electroporating the cells rested for at least 10 minutes while still in the cuvettes. A sample of cells without adding RNA was always electroporated as a mock control. After the electroporated cells rested in the cuvettes and were added to $9.6 \mathrm{ml}$ pre-warmed (in a $37^{\circ} \mathrm{C}$ water bath) media and plated onto $10 \mathrm{~cm}$ plates. The cells were incubated at $37^{\circ} \mathrm{C}$.

\subsubsection{Passaging experiments}

Cells were passaged as described above every 3 days or when $>80 \%$ confluent. Cell culture medium of the transfected cells was collected at various time points post-electroporation. Medium was clarified of cells by centrifugation at 3000rpm for 5 minutes.

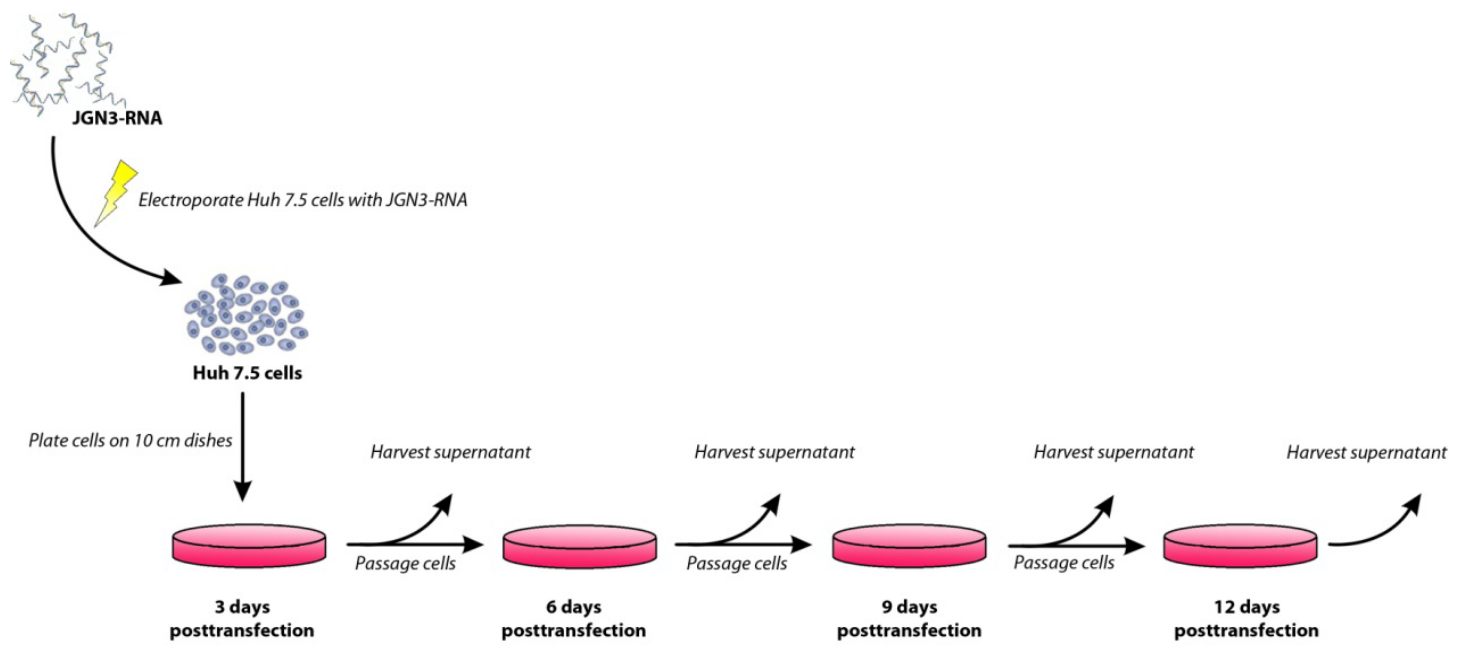

Figure 13 Passaging experiments. Huh7.5 Cells were electroporated with JGN3-RNA and plated on $10 \mathrm{~cm}$ dishes. When cells were about $80 \%$ confluent (about every 3 days) cell culture supernatant was harvested and the cells were passaged. The harvested supernatants were used for further analysis and were stored at $-80^{\circ} \mathrm{C}$. 


\subsubsection{GLuc activity/Luciferase assay}

For determination of replication a Gaussia Luciferase (GLuc) assay was performed with the BioLux $^{\mathrm{TM}}$ Gaussia Luciferase Kit. GLuc from the marine copepod Gaussia princeps is a reliable reporter to monitor gene-expression and replication via bioluminescence. FMDV 2A peptide mediates autocleavage of the protein at the GLuc2A/NS2 site.

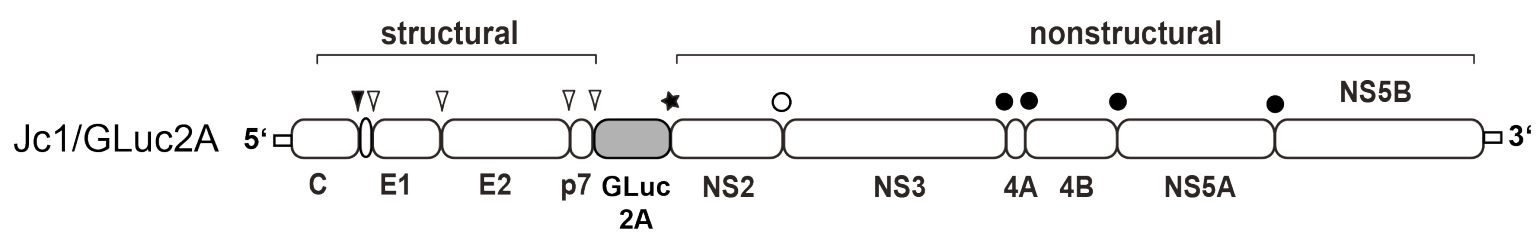

Figure $14 \mathrm{GLuc}$ is a sensitive reporter for virus replication and infectivity. Scheme of the HCV polyprotein and polyprotein processing with the GLuc 2A insertion. Signal peptidase cleavage site is shown by filled arrowheads, signal peptide peptidase cleavage sites by open arrowheads, the FMDV 2A autocleavage site is indicated by a filled star, NS2-NS3 cysteine autoprotease cleavage by an open circle and NS3-NS4A serine protease cleavage sites by filled arrowheads.

The used Jc1 replicons contain the GLuc gene. GLuc is expressed by the cells during the replication process and secreted to the cell culture supernatant. The luciferase catalyses an ATP-independent oxidation of the substrate coelenterazine. This reaction emits light (New England Biolabs 2011; Wiles et al. 2005; Tannous et al. 2005).
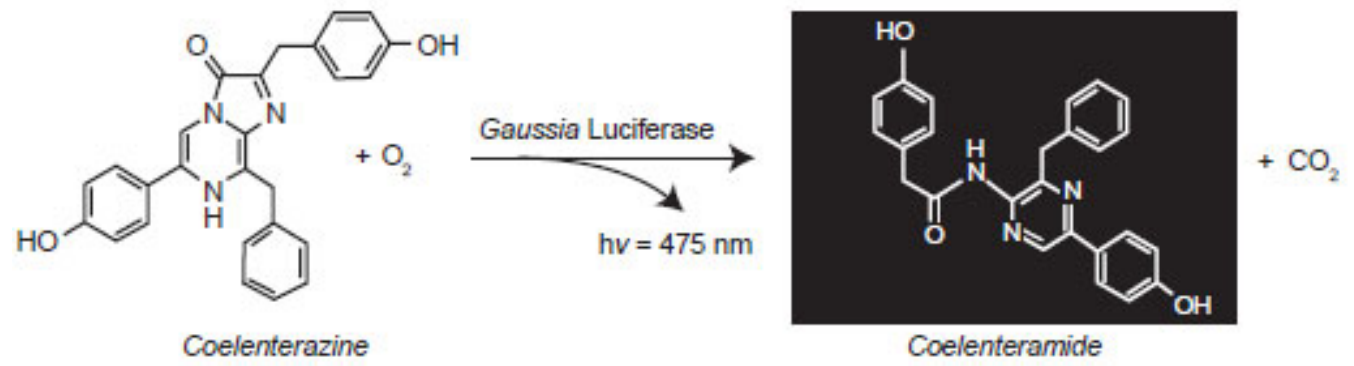

Figure 15 Luciferase catalyzed oxidation of Coelenterazine (New England Biolabs 2011). Luciferase was expressed and secreted by Jc1 transfected Huh 7.5 cells. In order to monitor replication luciferase activity was measured with the luminescent plate reader. Therefore a coelenterazine-buffer-mix was added to the sample. Secreted luciferase in cell culture supernatants react with coelenterazine which emits light at $475 \mathrm{~nm}$. This can be measured by the luminescent plate reader.

$80 \mu \mathrm{l}$ of collected cell culture medium was mixed with $20 \mu \mathrm{l}$ of Renilla $5 \mathrm{x}$ lysis buffer (Promega; Wisconsin, USA) in order to eliminate infectivity. $20 \mu$ l of this sample was used for GLuc activity measurement. BioLux ${ }^{\mathrm{TM}}$ GLuc Substrate was mixed 1:100 with BioLux ${ }^{\mathrm{TM}}$ GLuc Assay Buffer. On a Centro LB 960 luminescent plate reader (Berthold) the sample was injected with $50 \mu \mathrm{l}$ Gaussia luciferase assay reagent (New England Biolabs 2011) and measured with an integration time of $10 \mathrm{~s}$. 


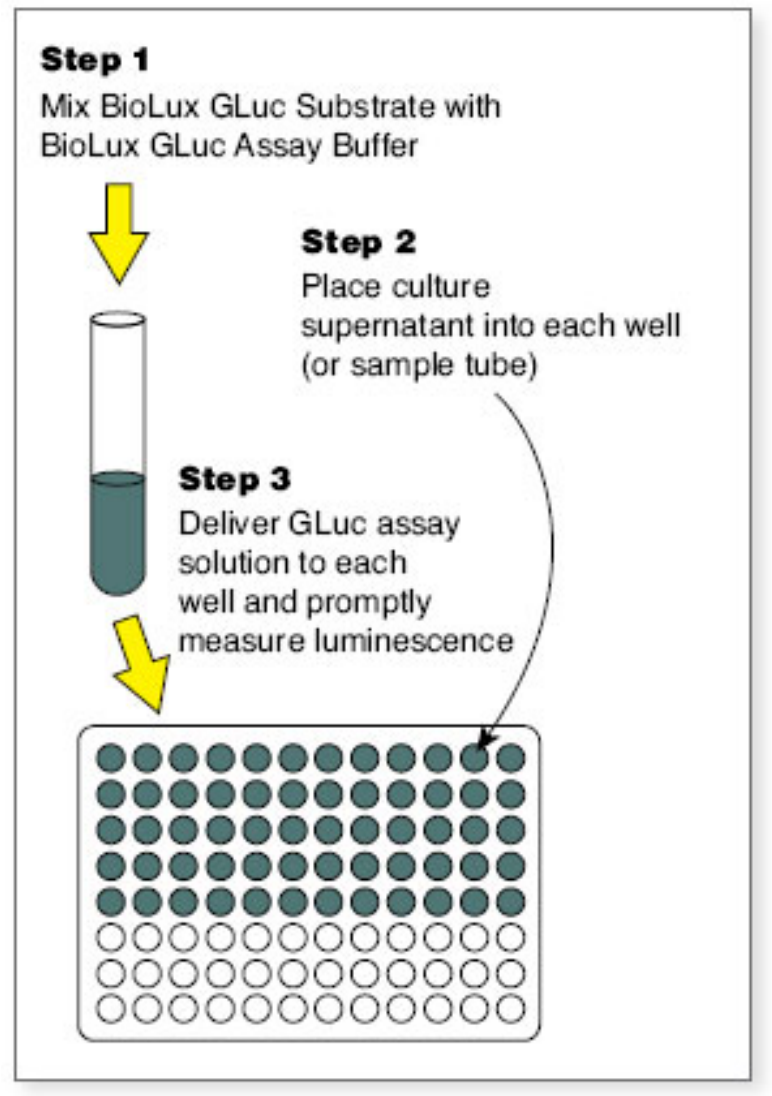

Figure 16 BioLux $^{\mathrm{TM}}$ GLuc Assay (New England Biolabs 2011). First, substrate and buffer were mixed 1:100. 20 $\mu$ of each sample was used per well. 50 $\mu$ l substrate-buffer-mix were delivered to each well and the measurement was done with a 10s integration time using the luminescent plate reader Berthold Centro LB 960.

\subsubsection{Infections}

For measuring infectivity of the different constructs, $20 \mu \mathrm{l}$ of the supernatants harvested at various time points were used to infect native Huh 7.5 cells. Therefore, native cells were seeded at $6.4 \times 10^{3}$ cells/well in $80 \mu \mathrm{l}$ media one day prior of the infectivity assay. Each well was coated with $30 \mu$ l Poly-L-lysine hydrobromide diluted to $100 \mu \mathrm{g} / \mathrm{ml}$ in $\mathrm{ddH}_{2} \mathrm{O}$.

After a $24 \mathrm{~h}$ absorption period the cells were washed three times with Dulbecco's PBS and $100 \mu \mathrm{l}$ media was added. The cells were incubated at $37^{\circ} \mathrm{C}$ for another $48 \mathrm{~h}$. Afterwards media was collected and a Luciferase assay was performed as described above.

\subsubsection{Intracellular Infectivity}

To measure intracellular infectivity cells were harvested $48 \mathrm{~h}$ post-transfection by trypsinization followed by centrifugation at 1200rpm for 5 minutes. The cells were resuspended in a small volume of complete media and snap-frozen in liquid nitrogen. In order to disrupt the cells three rounds of thawing $\left(37^{\circ} \mathrm{C}\right)$ and refreezing in liquid nitrogen 
$\left(-196^{\circ} \mathrm{C}\right)$ were performed. Cellular debris was removed by centrifugation at $3000 \mathrm{rpm}$ for 5 minutes. The supernatants were tested for infectivity as described above.

\subsubsection{Anti-NS5a staining of HCV-infected Huh 7.5 cells}

In order to measure infectivity besides the Luciferase assay anti-NS5a staining was used. Therefore $72 \mathrm{~h}$ post-infection media was removed from the plates with the infected cells and washed with1xPBS. To fix the cells PBS was replaced by ice-cold methanol and incubated in the $-20^{\circ} \mathrm{C}$ freezer for 20 minutes to several days. Afterwards, the cells do not contain infectious material any more. Methanol got dumped off and the plates were air-dried for at least 10 minutes until completely dry. Then the plates were washed twice with 1xPBS and endogenous peroxidase was blocked by adding 1 xPBS containing $3 \% \mathrm{H}_{2} \mathrm{O}_{2}$ for 5 minutes at room temperature. The plates were washed twice again with 1xPBS and blocking buffer was added. The plates were blocked for 30 minutes with gently rocking at room temperature. The blocking buffer was replaced by PBS-T containing a 1:20000 dilution of anti-NS5a monoclonal antibody 9E10. The plates were incubated 1 hour at room temperature with gentle rocking and washed twice with 1xPBS afterwards. The secondary antibody (ImmPress anti-mouse-HRP) was diluted two-fold in 1xPBS-T, added to the plates and incubated for 30 minutes at room temperature with gentle rocking. Then the plates were washed twice with 1XPBS and once with 1XPST-T. In order to develop the plates Immpact DAB was used according to the manufactures instructions. The plates were incubated at room temperature for 1 to 10 minutes. The stained plates were analyzed using an inverted light microscope (Nikon).

\subsubsection{RNA extraction}

RNA extraction from harvested supernatants was performed using the 'Quiagen viral RNA extraction kit' after the manufacturer's instructions.

\subsubsection{Quantitative RT-PCR}

Quantitative RT-PCR is a reliable and very sensitive method for detection and quantization of nucleic acids levels. The qRT-PCR machine is able to perform real time detection of PCR products as they accumulate during PCR cycles.

For the detection of HCV positive strand RNA specific primers and minor groove binding (MGB) probe were used. Primers target a conserved region of the HCV 5' untranslated region (UTR) from genotype $2 a$. 


\begin{tabular}{ll}
\hline Forward primer & 5'-CTTCACGCAGAAAGCGTCTA-3' \\
\hline Reverse primer & 5'-CAAGCACCCTATCAGGCAGT-3' \\
\hline Probe & 5'-6FAM-TATGAGTGTCGTGCAGCCTC-MGBNFQ-3' \\
\hline Table 12 Sequence of primers and probe used for RT-PCR
\end{tabular}

The specific probe is label with the fluorescent reporter dye 6-Carboxyflourescein (6-FAM) at the 5' end and the minor groove binding non-fluorescent quencher (MGBNFQ) at the 3' end. In an intact probe the reporter dye emission is quenched by the non-fluorescent quencher. During each PCR cycle the DNA polymerase cleaves the 5' reporter dye from the probe through its 5'-3' nuclease activity. The reporter dye gets released and emits its characteristic fluorescence. The change in the emitted fluorescence correlates with the amount of PCR product produced.

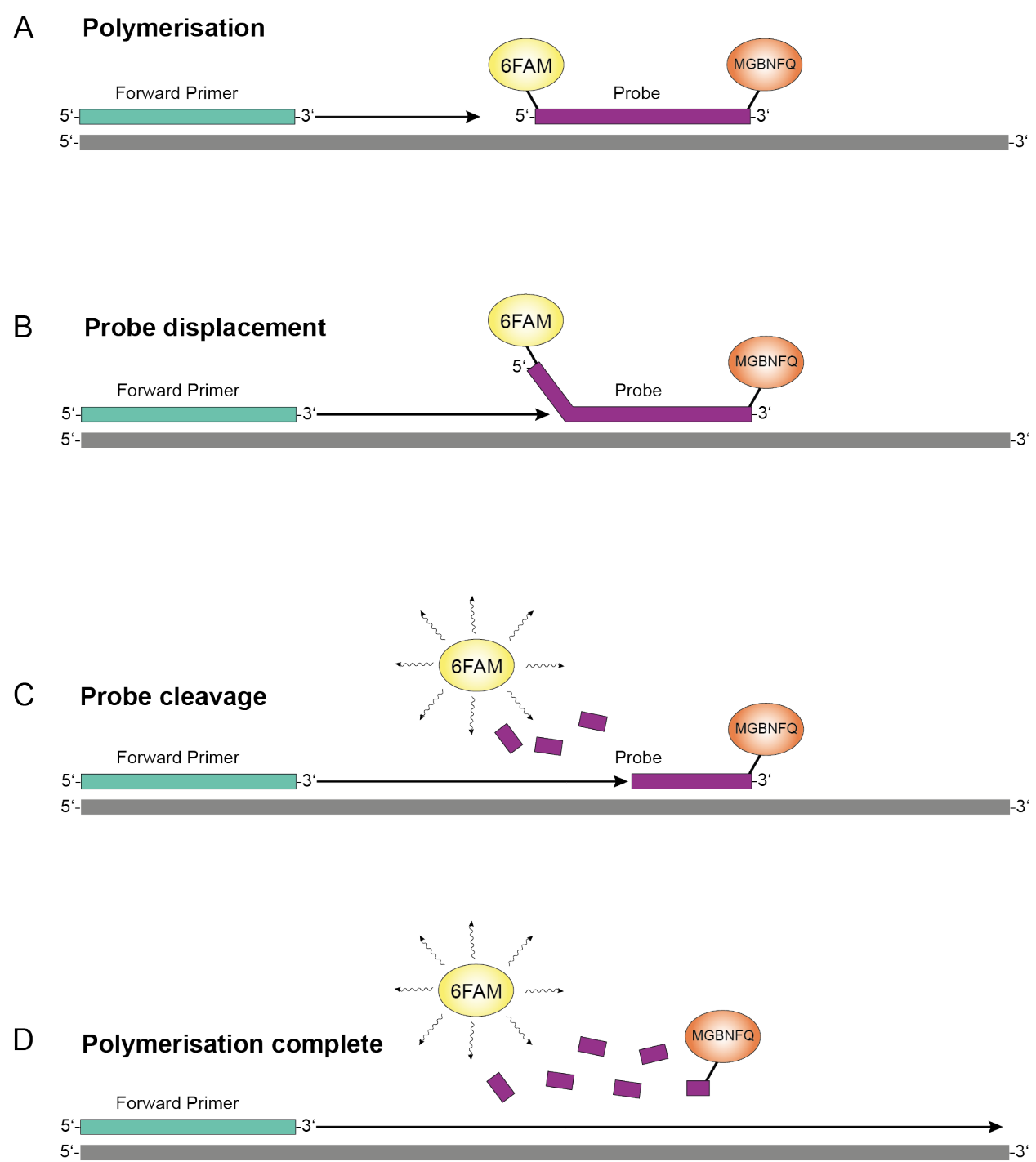

Figure 17 Scheme of RT-PCR reaction. 
Quantitative RT-PCR was performed by using the LightCycler RNA Amplification Kit with the Roche LightCycler 480. All mixing was performed in an Air Clean 600 PCR Workstation.

The master mix was prepared as follows:

$\begin{array}{ll}7.55 \mu \mathrm{l} & \text { Nuclease-free water } \\ 4 \mu \mathrm{l} & \text { Reaction Mix } \\ 4 \mu \mathrm{l} & \mathrm{MgCl}_{2} \\ 0.75 \mu \mathrm{l} & \text { Forward Primer } \\ 0.75 \mu \mathrm{l} & \text { Reverse Primer } \\ 0.5 \mu \mathrm{l} & \text { Probe } \\ 0.4 \mu \mathrm{l} & \text { Enzyme Mix } \\ 0.05 \mu \mathrm{l} & \text { RNase Inhibitor }\end{array}$

A $1: 10$ dilution series of the standard was made to create determinants ranging from $4 \times$ $10^{7}$ copies per reaction to $4 \times 10^{0}$ copies per reaction.

$2 \mu \mathrm{l}$ of HCV standard, unknown sample or no template control were added to $18 \mu \mathrm{l}$ of the master mix in the qRT-PCR 96 well plate. After loading the master mix and samples a sealing foil cover was placed on the plate and the plate was spun at 1000rpm for a few minutes. The plate was placed in the Roche LightCycler 480 using the following cycle conditions:

\begin{tabular}{llll}
\hline FastTaq Trial & Temperature $\left({ }^{\circ} \mathrm{C}\right)$ & Hold & Ramp Rate $\left({ }^{\circ} \mathrm{C}\right)$ \\
\hline RT & 56 & $45 \mathrm{~min}$ & 4.4 \\
\hline Denaturation & 95 & $5 \mathrm{~min}$ & 4.4 \\
\hline PCR (50 cycles) & 95 & $10 \mathrm{sec}$ & 4.4 \\
\hline & 60 & $20 \mathrm{sec}$ & 2.2 \\
\hline & 72 & $3 \mathrm{sec}$ & 4.4
\end{tabular}

Table 13 LightCycler conditions 


\subsubsection{Pulldown experiments}

For these experiments Jc1 constructs expressing biotin acceptor peptide (BAP) at the aminoterminal end of NS2 were used to determine physical interactions between proteins important for virus assembly. Huh 7.5 cells which express the E.coli biotin ligase BirA were transfected with Jc1/bioNS2 RNA.

Expression of biotin AP-tagged proteins in the presence of E.coli biotin ligase BirA results in the addition of a biotin molecule on the lysine residue of the BAP.

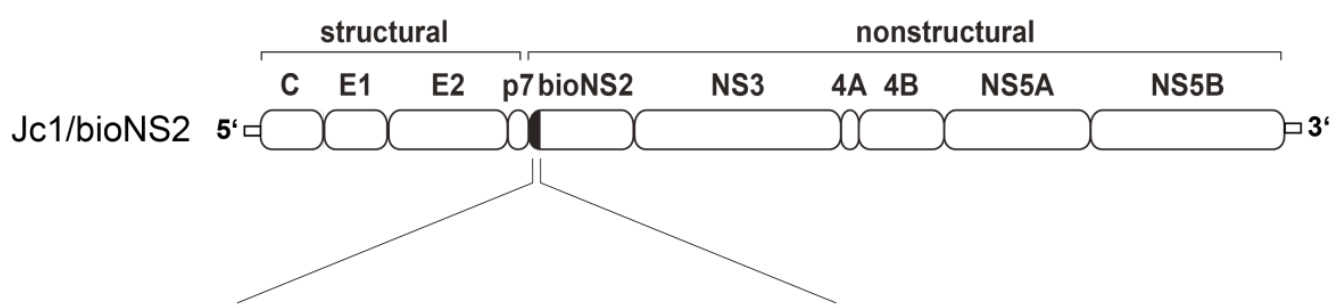

...AYAYDAPGLNDIFEAQKIEWHGGS...

biotin AP
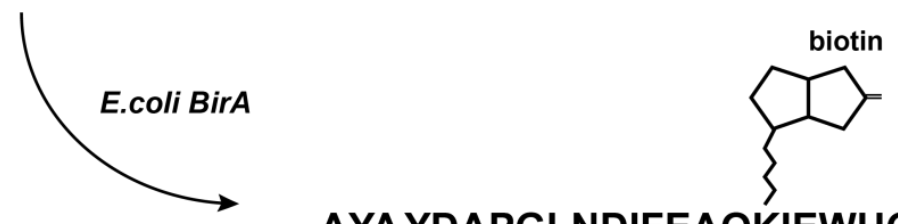

...AYAYDAPGLNDIFEAQKIEWHGGS...

biotin AP

Figure 18 Biotinylation of the biotin acceptor peptide (BAP) catalyzed by E.coli BirA.

For isolation of biotinylated proteins Streptavidin-coupled Dynabeads ${ }^{\mathrm{TM}} \mathrm{M}-280$ were used. Streptavidin binds biotin molecules with high affinity. Biotinylated proteins get bound to the magnetic beads. The beads can get isolated with magnetic forces using a magnetic rack and proteins can be purified from total cell lysates.

48h post-transfection the cells were washed with 1x PBS and trypsinized. Cells were harvested and centrifuged at 1200rpm at $4^{\circ} \mathrm{C}$ for 6 minutes. Afterwards cells were washed twice with $5 \mathrm{ml}$ PBS. Then the cells were resuspended in $1 \mathrm{ml}$ TBS-Tx and put on ice for 30 minutes to completely lyse the cells.

The lysate was spun in a microfuge at $13,000 \mathrm{rpm}$ at $4^{\circ} \mathrm{C}$ for 5 minutes to remove cellular debris. The supernatant was collected and placed in a fresh $2 \mathrm{ml}$ tube. $20 \mu \mathrm{l}$ of the lysate was stored at $-80^{\circ} \mathrm{C}$ as the total contents control sample for later experiments.

To remainder of lysate $50 \mu$ l of prewashed streptavidin magnetic beads were added. Per sample $50 \mu \mathrm{l}$ beads were prewashed with $167 \mu \mathrm{l}$ buffer TBS-Tx on a nutator at $4^{\circ} \mathrm{C}$ for $30 \mathrm{~min}$. 
Supernatant was removed and the beads were resuspended in $50 \mu \mathrm{I}$ TBS-Tx per sample. The lysate/magnetic beads mix was incubated on the nutator at $4^{\circ} \mathrm{C}$ for $1 \mathrm{~h}$. Afterwards the mix was separated on a magnetic tube rack (MagnaRack). The supernatant was removed and $1 \mathrm{ml}$ TBS-Tx was added to the samples and nutated for another $15 \mathrm{~min}$. This washing step was repeated two more times.

Then $100 \mu$ l of protein sample was added and samples were heated to $94^{\circ} \mathrm{C}$ for $10 \mathrm{~min}$. Samples were spun briefly and placed on the MagnaRack to separate proteins from magnetic beads. Supernatants were removed. An equal volume of protein sample buffer was added to these supernatants and to the controls. Controls were heated to $94^{\circ} \mathrm{C}$ for 10 minutes as well and all samples were separated by sodium dodecylsulfate polyacrylamide electrophoresis (SDS-PAGE) on a $12 \%$ SDS-PAGE gel and transferred to PVDF membranes. 
A

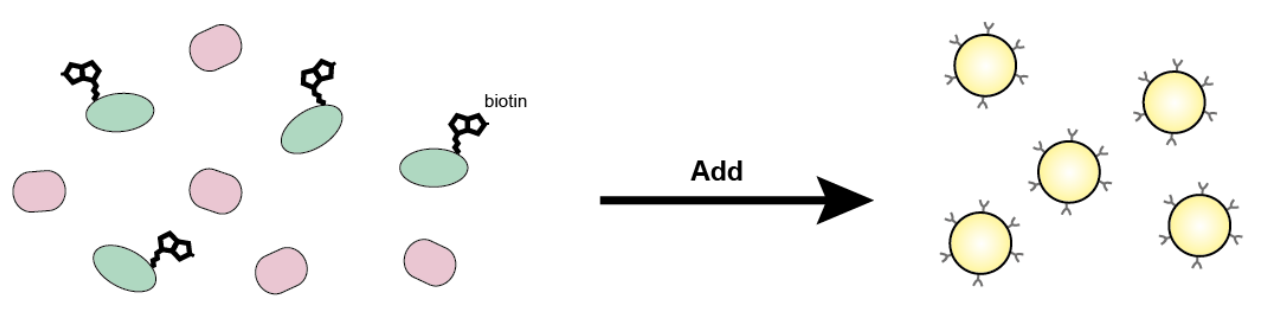

Total cell lysate including biotinylated proteins

Streptavidin magnetic beads

B

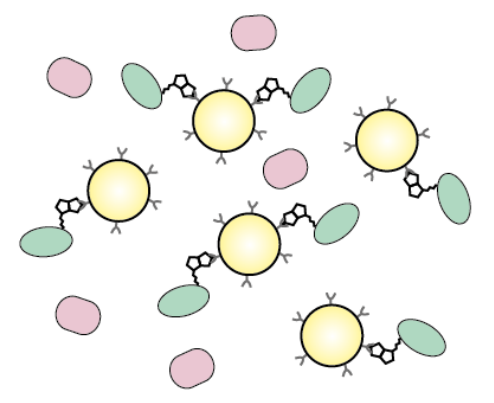

C

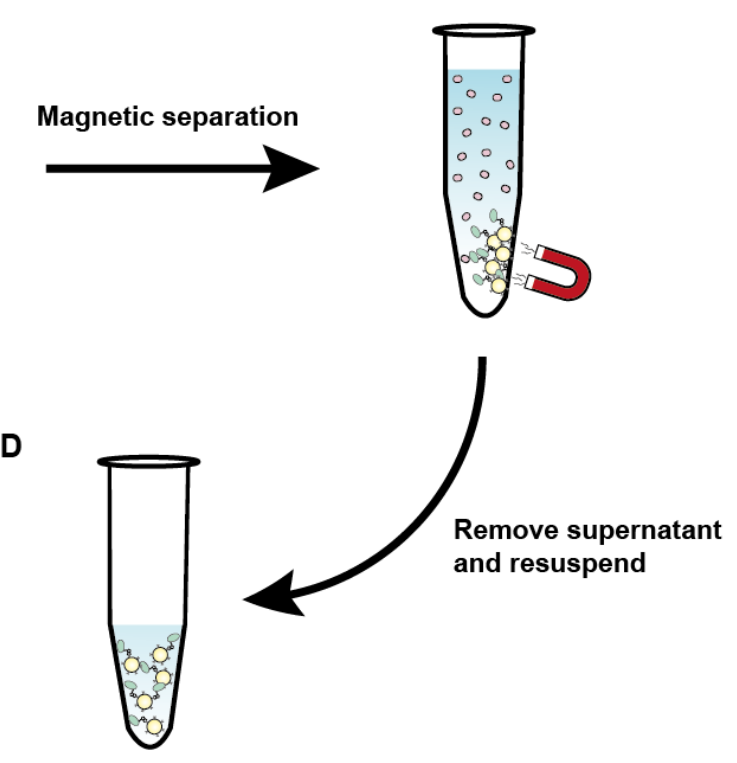

E

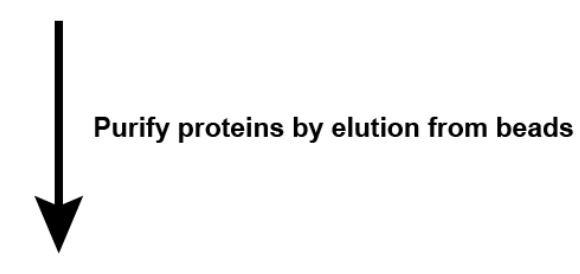

Figure 19 Pulldown of biotinylated proteins using Streptavidin magnetic beads. 


\subsubsection{Western blot}

Western blot was performed after standard methods. Samples were prepared in protein sample buffer, separated on a $12 \%$ SDS-PAGE gel as described above.

Proteins were transferred to a PVDF membrane in transfer buffer running at $110 \mathrm{~V}$ for $1 \mathrm{~h}$. The membrane was blocked in blocking buffer $\left(5 \%\right.$ milk) for $1 \mathrm{~h}$ at RT or overnight at $4^{\circ} \mathrm{C}$. Then the membrane was probed with the antibody against NS2 diluted 1:5000 in blocking buffer for $1 \mathrm{~h}$ at $\mathrm{RT}$ or at $4^{\circ} \mathrm{C}$ overnight. The membrane was washed a couple of times with PBS-T and incubated with the secondary anti-mouse antibody diluted 1:5000 in blocking buffer for $1 \mathrm{~h}$ followed by another couple of washes with PBS-T. For the development the SuperSignal kit was used. 


\section{Results}

\subsection{Confirmation of pJGN3 clones}

Genotypes of the Jc1/Gluc2 clones had to be confirmed first. Correct mutations in the NS3hel domain had to be verified.

The correct insertion of the mutations was checked by digestion with restriction enzymes EcoRI and BsrGI. Fragments were separated on a $0.8 \%$ agarose gel and compared to the maternal pJRB restriction fragments as well as to the vector fragments ( $p J c 1)$.
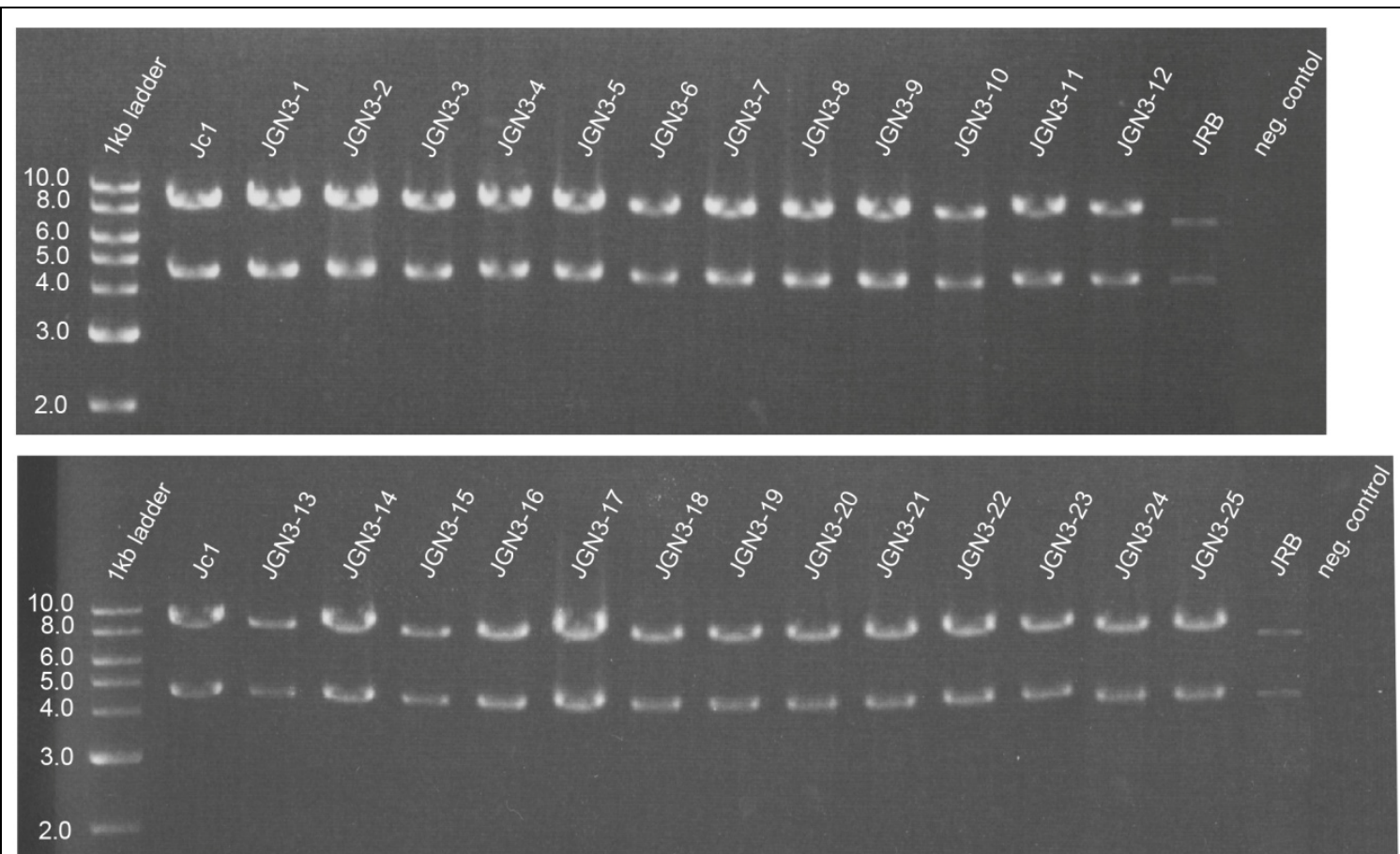

\begin{tabular}{|l|l|l|}
\hline Construct & $\begin{array}{l}\text { Expected size of bigger } \\
\text { fragment }[k b]\end{array}$ & $\begin{array}{l}\text { Expected size of smaller } \\
\text { fragment [kb] }\end{array}$ \\
\hline JRB & 7800 & 4564 \\
\hline JGN3 & 8382 & 4564 \\
\hline JC1 & 8382 & 4564 \\
\hline
\end{tabular}

Figure 20 Agarose gel of restriction fragments. Expected size of the bigger fragments of pJGN3 is bigger than the one of pJRB. Smaller fragments are expected to be equal in size. Smaller fragments include the mutations. Expected fragment sizes of pJGN3 are equal to pJc1/Gluc2.

All fragments appeared at the expected sizes in the agarose gel. 
In addition, the correct introduction of the NS3hel mutations was confirmed by sequencing the plasmids at the W.M. Keck Foundation Biotechnology Resource Center at Yale University.

1801...CGTTTGGGCCCTATTACCAATGAGGTCACCCTCACACACCCTGGGACGAAGTACATCGCC...1860 ...CGTTTGGGCCCTATTACCAATGAGGTCACCCTCGCCGCCGCCGGGACGGCCGCCATCGCC... ...CGTTTGGGCCCTATTACCAATGAGGTCACCCTCGCCGCCCCTGGGACGAAGTACATCGCC... ...CGTTTGGGCCCTATTACCAATGAGGTCACCCTCACACACGCCGGGACGGCCTACATCGCC... ...CGTTTGGGCCCTATTACCAATGAGGTCACCCTCACACACCCTGGGACGAAGGCCATCGCC...

Jc1 JGN3-1 JGN3-2 JGN3-3 JGN3-4

Jc1 JGN3-5 JGN3-6 JGN3-7 ...ATGTGGAAGTGCCTGGCCCGACTCAAGGCCGCCCTTGCGGGCCCCACACCTCTCCTGTAC...

1501...TGGTACGATCTCACACCAGCGGAGACCACCGTCAGGCTTAGAGCGTATTTCAACACGCCC...1560 ...TGGTACGCCCTCACACCAGCGGCCACCACCGTCGCCCTTAGAGCGTATTTCAACACGCCC... ...TGGTACGCCCTCACACCAGCGGAGACCACCGTCAGGCTTAGAGCGTATTTCAACACGCCC... ...TGGTACGATCTCACACCAGCGGCCACCACCGTCGCCCTTAGAGCGTATTTCAACACGCCC...

Jc1 JGN3-8 JGN3-9 JGN3-10

1564...CTACCCGTGTGTCAAGACCATCTTGAATTTTGGGAGGCAGTTTTCACCGGCCTCACACAC...1623 ...CTACCCGTGTGTCAAGACCATCTTGCCTTTTGGGCCGCAGTTTTCACCGGCCTCACACAC... ...CTACCCGTGTGTCAAGACCATCTTGAATTTTGGGAGGCAGTTTTCGCCGGCCTCGCCGCC...

Jc1 JGN3-11 JGN3-12

1621...CACATAGACGCCCACTTCCTCTCCCAAACAAAGCAAGCGGGGGAGAACTTCGCGTACCTA...1680 ...CACATAGCCGCCGCCTTCCTCGCCCAAACAAAGGCCGCGGGGGAGAACTTCGCGTACCTA... ...CACATAGCCGCCGCCTTCCTCTCCCAAACAAAGCAAGCGGGGGAGAACTTCGCGTACCTA... ...CACATAGACGCCCACTTCCTCGCCCAAACAAAGGCCGCGGGGGAGAACTTCGCGTACCTA...

$\mathrm{JC} 1$ JGN3-13 JGN3-14 JGN3-15

1021...CTCGGGCGGGAGGGTGAGATCCCCTTCTATGGGAGGGCGATTCCCCTATCCTGCATCAAG...1080 ...CTCGGGCGGGAGGGTGCCATCGCCGCCGCCGGGAGGGCGATTGCCCTATCCTGCATCAAG... ...CTCGGGCGGGAGGGTGCCATCGCCTTCTATGGGAGGGCGATTCCCCTATCCTGCATCAAG... ...CTCGGGCGGGAGGGTGAGATCCCCGCCTATGGGAGGGCGATTGCCCTATCCTGCATCAAG... ...CTCGGGCGGGAGGGTGAGATCCCCTTCGCCGGGAGGGCGATTCCCCTATCCTGCATCAAG... ...CTCGCCGCTGCAGGTGAGATCCCCTTCTATGGGAGGGCGATTCCCCTATCCTGCATCAAG...

901...CTCGGCATCGGAACGGTCCTTGATCAAGCAGAGACAGCCGGGGTCAGACTAACTGTGCTG...960 ...CTCGGCATCGGAACGGTCCTTGCCCCTGCAGCCGCCGCCGGGGTCAGACTAACTGTGCTG... ...CTCGGCATCGGAACGGTCCTTGCCCCTGCAGAGACAGCCGGGGTCAGACTAACTGTGCTG... ...CTCGGCATCGGAACGGTCCTTGATCAAGCAGCCGCCGCCGGGGTCAGACTAACTGTGCTG...

609...GCTCCAACTGGCAGTGGAAAGAGCACCAAGGTCCCTGTCGCGTATGCCGCCCAGGGGTAC...669 ...GCTCCAACTGGCAGTGGAAAGAGCACCAAGGTCCCTGTCCGCTATGCCGCCCAGGGGTAC... ...GCTCCAACTGGCAGTGGAAAGAGCACCAAGGTCCCTGTCCGCTATGCCGCCGCCGCCGCCC...
$\mathrm{Jc} 1$

JGN3-16

JGN3-17

JGN3-18

JGN3-19

JGN3-20

Jc1

JGN3-21

JGN3-22

JGN3-23

Jc1

JGN3-24

JGN3-25

Figure 21 Verified NS3 sequences. The sequence of Jc1 is shown in top lines. Mutations are marked underlined and red. Numbers in each top line show positions within the NS3 region. Numbers are in bases. Numbering of the NS3 sequence is relative to the sequence of the NS3 region of full length replicon Jc1. 
In all 25 plasmids the mutations of interest were correctly inserted within the NS3hel domain of pJc1/Gluc2. Therefore the plasmids were correct and could be used for the following experiments.

\subsection{Replication}

All mutants were tested for their ability to replicate in cell culture. In order to study HCV virion assembly only those mutants showing the ability to efficiently replicate can be used for further studies. Furthermore one can learn about residues essential for replication within the NS3hel domain that were not mapped yet.

\subsubsection{Constructs with changes within NS3hel domain 3}

15 constructs with various alanine substitutions within the NS3 subdomain 3 were made by cloning. Four of them have changes within a well conserved protuberance at the side of domain 3 which projects from alpha helix 21 . Three constructs contain alanine substitutions in a well conserved corner of domain 3 within alpha helix 20 , another three in a well conserved acidic patch on the surface of domain 3 which projects from alpha helix 14 . Two more mutants have changes in well conserved residues projecting from alpha helix 15 and three more constructs in a well conserved patch on the surface of domain 3 , projecting from alpha helix 16 (see figure 22).

A

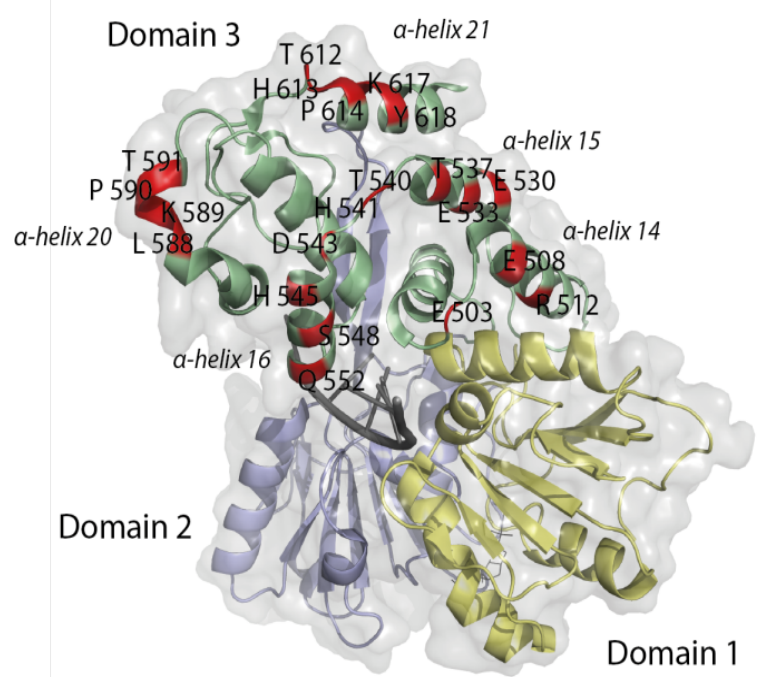

B

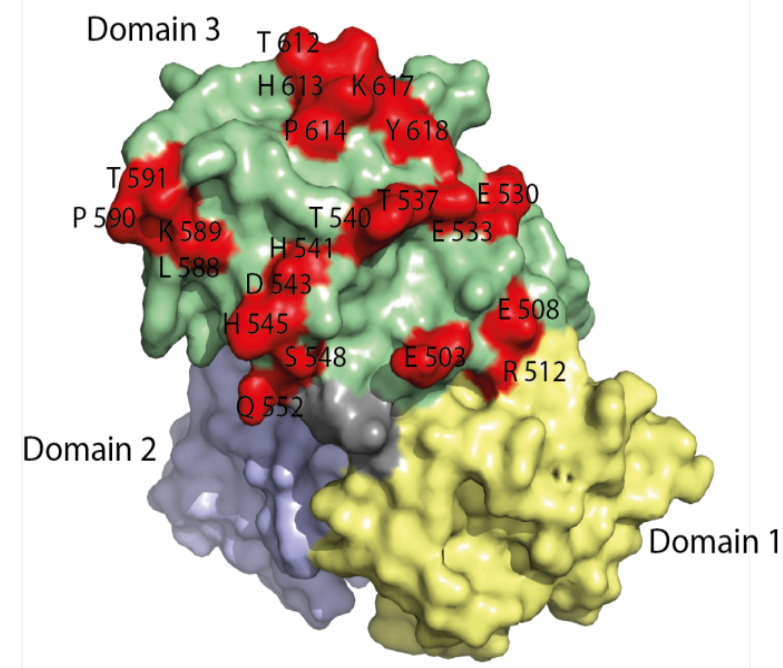

Figure 22 Locations of mutations within NS3hel domain 3. (A) Ribbon model of NS3hel. Domain 1, domain 2 and domain 3 are colored yellow, light blue and green. Substituted residues are highlighted red and marked by 1-letter-code residue name and position. (B) Surface model of NS3hel. Mutated residues are highlighted red. Models were rendered with PyMOL; Protein Data Bank code 3KQL was used. 


\begin{tabular}{|c|c|c|}
\hline Name of construct & Mutation & Location of Alanine Substitution \\
\hline \multirow[t]{5}{*}{ JGN3-1 } & T612A & \multirow{10}{*}{$\begin{array}{l}\text { well conserved protuberance from the side of } \\
\text { domain } 3\end{array}$} \\
\hline & $\mathrm{H} 613 \mathrm{~A}$ & \\
\hline & P614A & \\
\hline & K617A & \\
\hline & Y618A & \\
\hline \multirow[t]{2}{*}{ JGN3-2 } & T612A & \\
\hline & $\mathrm{H} 613 \mathrm{~A}$ & \\
\hline \multirow[t]{2}{*}{ JGN3-3 } & P614A & \\
\hline & K617A & \\
\hline JGN3-4 & Y618A & \\
\hline \multirow[t]{4}{*}{ JGN3-5 } & L588A & \multirow{8}{*}{$\begin{array}{l}\text { well conserved corner of domain } 3 \text { within } \\
\text { alpha helix } 20\end{array}$} \\
\hline & K589A & \\
\hline & P590A & \\
\hline & T591A & \\
\hline \multirow[t]{2}{*}{ JGN3-6 } & L588A & \\
\hline & K589A & \\
\hline \multirow[t]{2}{*}{ JGN3-7 } & P590A & \\
\hline & T591A & \\
\hline \multirow[t]{3}{*}{ JGN3-8 } & D503A & \multirow{3}{*}{$\begin{array}{l}\text { well conserved acidic patch on the surface of } \\
\text { domain } 3\end{array}$} \\
\hline & E508A & \\
\hline & R512A & \\
\hline JGN3-9 & D503A & projecting from alpha helix 14 \\
\hline \multirow[t]{2}{*}{ JGN3-10 } & E508A & \\
\hline & R512A & \\
\hline \multirow[t]{2}{*}{ JGN3-11 } & E530A & \multirow{5}{*}{$\begin{array}{l}\text { well conserved residues } \\
\text { projecting from alpha helix } 15\end{array}$} \\
\hline & E533A & \\
\hline \multirow[t]{3}{*}{ JGN3-12 } & T537A & \\
\hline & T540A & \\
\hline & $\mathrm{H} 541 \mathrm{~A}$ & \\
\hline \multirow[t]{4}{*}{ JGN3-13 } & D543A & \multirow{8}{*}{$\begin{array}{l}\text { well conserved patch on the surface of domain } \\
3 \\
\text { projecting from alpha helix } 16\end{array}$} \\
\hline & H545A & \\
\hline & S548A & \\
\hline & Q552A & \\
\hline \multirow[t]{2}{*}{ JGN3-14 } & D543A & \\
\hline & $\mathrm{H} 545 \mathrm{~A}$ & \\
\hline \multirow[t]{2}{*}{ JGN3-15 } & S548A & \\
\hline & Q552A & \\
\hline
\end{tabular}

Table 14 Changes within NS3hel domain 3.

To determine replication of JGN3-constructs in the context of HCV full length replicon Jc1 in cell culture passaging experiments were performed on the basis of the Jc1/GLuc2 reporter system as described in 'Materials and Methods' (p. 37ff.). Briefly, Huh 7.5 cells were 
transfected with RNA of the different mutants. Cells were split after $72 \mathrm{~h}$ or when subconfluent. The GLuc gene and the FMDV auto-cleavage peptide 2A were encoded between $\mathrm{p} 7$ and NS2. GLuc2 was secreted into the cell culture medium of Huh 7.5 transfected cells reporting replication activity as GLuc activity could be measured over time. Cell culture media was harvested every $24 \mathrm{~h}$ and GLuc activity was measured as described in Material and Methods. Functionality and sensitivity of this system were described earlier by Phan et al. (2009). As a control the polymerase defective construct Jc1/GLuc2(GNN) was used to compare values to a construct that is known to be unable to replicate as it lacks polymerase activity. Jc1/GLuc2( $\Delta$ core) was used to monitor a construct which is able to replicate but unable to produce infectious virus as it lacks the core region. Jc1/GLuc2( $\Delta$ core) shows around 10 fold lower levels of secreted GLuc activity than WT values due to its defect in production of infectious virus particles which makes it unable to spread within the cell population. Furthermore wild-type Jc1/GLuc2 (WT) and mock controls were used.

\subsubsection{Replication of constructs with changes within NS3hel domain 3 alpha helix 20 and 21}

As expected, WT Jc1/GLuc2 efficiently replicated. Measured GLuc activity representing replication was $5.7 \times 10^{4} \mathrm{RLU}$ after 24 hours post-transfection rising to $2.4 \times 10^{6} \mathrm{RLU}$ after 48 hours to $9.6 \times 10^{8} \mathrm{RLU}$ and $6.8 \times 10^{7} \mathrm{RLU}$ at 72 hour and 96 hour time points. Jc1/GLuc2(Acore) also replicated but showed, as described above, more than 10 fold lower GLuc activity levels than WT. Polymerase defective Jc1/GLuc2(GNN) as well as mock control did not replicate. Mock GLuc levels remained around $2 \times 10^{3} \mathrm{RLU}$ at all time points. Jc1/GLuc2(GNN) GLuc activities stagnated around $4 \times 10^{4} \mathrm{RLU}$ during all measured time points post-transfection.

Within $\alpha$-helix 20 and 21 of NS3hel domain 3 three JGN3 constructs - JGN3-2, JGN3-3 and JGN3-5 - showed GLuc activity levels comparable to WT levels at $24 \mathrm{~h}, 48 \mathrm{~h}, 72 \mathrm{~h}$ as well as 96h post-transfection indication efficient replication. Mutations T612A and H613A (JGN3-2) had no impact on replication. Measured GLuc levels were similar to WT values at each time point. GLuc activities got up from $2.8 \times 10^{4} \mathrm{RLU} 24 \mathrm{~h}$ post-transfection to top values of $2.6 \times 10^{7}$ and $8.6 \times 10^{7} \mathrm{RLU}$ at $72 \mathrm{~h}$ and $96 \mathrm{~h}$ time-points. Alanine substitutions P614A and K617A (JGN3-3) did not influence replication either. Measured GLuc activity 24 hours posttransfection was $1.3 \times 10^{6} \mathrm{RLU}$ rising to $6.4 \times 10^{7} \mathrm{RLU}$ and $2.7 \times 10^{8} \mathrm{RLU} 72$ and 96 hours posttransfection. Phenotype of mutations L588A, K589A, P590A and T591A together (JGN-5) was also similar to WT Jc1/GLuc2 with values of $6.3 \times 10^{7} \mathrm{RLU}$ and $3.9 \times 10^{7} \mathrm{RLU}$ at 72 and 96 hour time points.

Mutations T612A, H613A, P614A, K617A and Y618A within the well conserved protuberance of $\alpha$-helix 21 lead when combined (JGN3-1) to a severely defect in replication. Values ranged between $4 \times 10^{4}$ and $2.7 \times 10^{5} \mathrm{RLU}$, around 1000 fold lower than WT GLuc activities. 
Furthermore, constructs JGN3-4, JGN3-5 and JGN3-7 showed reduced GLuc activities compared to WT. Single mutation Y618A (JGN-4) influenced replication. Values at 72 hours and 96 hours were with $2.1 \times 10^{6}$ and $4.4 \times 10^{6}$ RLU around 10 to 100 fold lower than WT measurements. Isolated mutations L588A with K589A (JGN-6) enhance GLuc activity from $1.4 \times 10^{5}$ at 24 hours post-transfection to $9.3 \times 10^{6} \mathrm{RLU}$ at 48 hours post-transfection. Replication activity drops to $1.4 \times 10^{6}$ and $3.5 \times 10^{5} \mathrm{RLU}$ at later time points 72 and 96 hours post-transfection. Mutations P590A and T591A (JGN-7) showed to increase GLuc activity from $4.4 \times 10^{4}$ to $5.9 \times 10^{6} \mathrm{RLU}$ from 24 hours to 96 hours post-transfection. But GLuc levels were around 10 fold lower than WT levels.

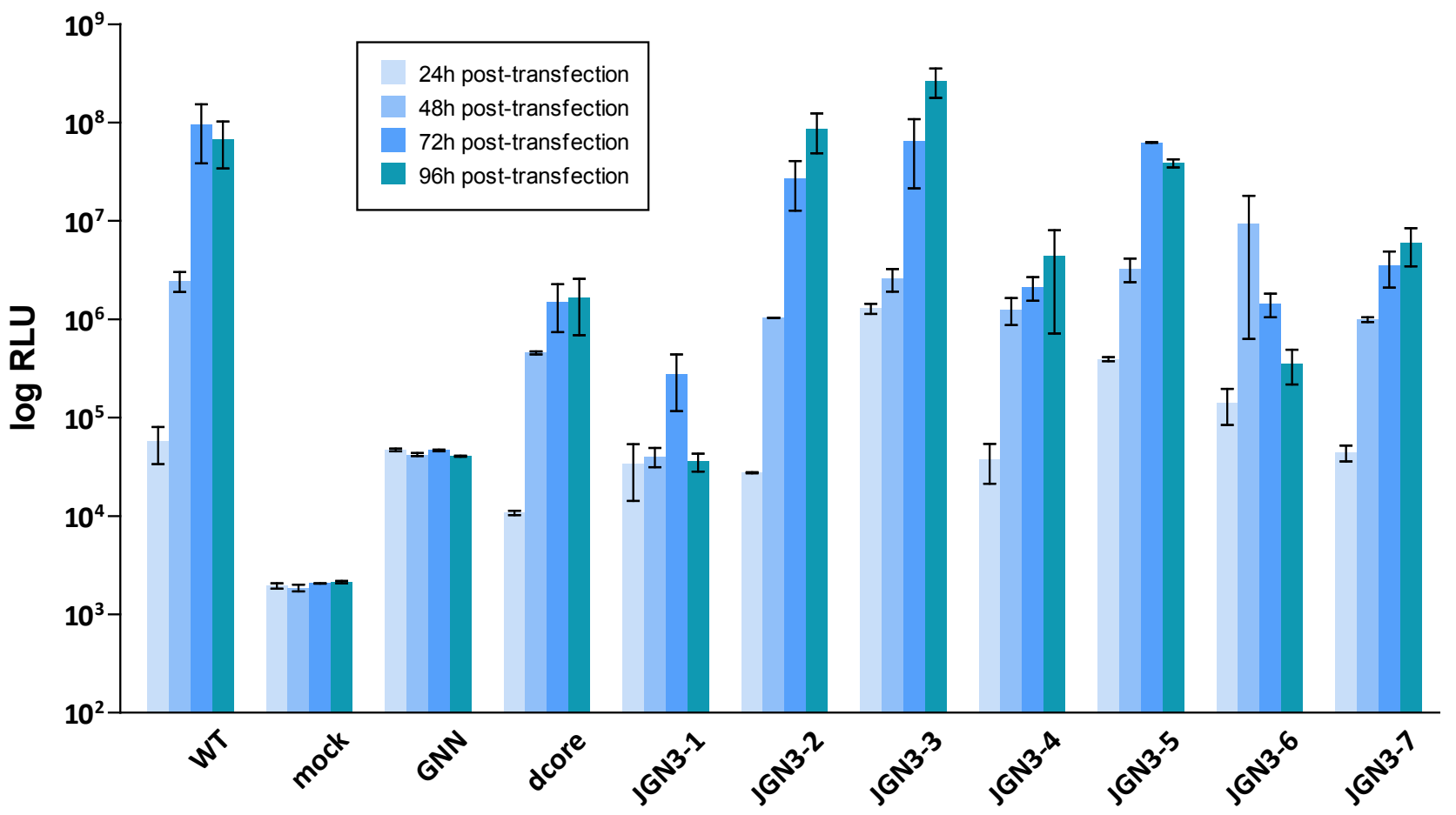

Figure 23 Replication of JGN3 constructs with changes in alpha helices $\mathbf{2 0}$ and $\mathbf{2 1}$ of domain $\mathbf{3}$. Cells were transfected with described mutants and controls. Medium was collected $24 \mathrm{~h}, 48 \mathrm{~h}, 72 \mathrm{~h}$ and $96 \mathrm{~h}$ post-transfection. Data represents secreted GLuc activity over time in Relative Light Units (RLU). Wild-type Jc1 (WT) is shown as a positive control. Further controls mock, GNN and $\Delta$ core (described above) are also shown. Values represent average measurements of at least two to three individual rounds of experiments. Error bars represent standard deviation of the mean.

\subsubsection{Replication of constructs with changes within NS3hel domain 3 alpha helix 14, 15} and 16.

Within a-helix 14, 15 and 16 of NS3hel domain 3 five constructs - JGN3-8, JGN3-9, JGN310, JGN3-12 and JGN3-15 - efficiently replicated showing GLuc activities alike WT. Combined mutations D503A, E508A and R512A within the acidic patch and $\alpha$-helix 14 (JGN8) were able to replicate like WT. GLuc activity levels raised from $6.4 \times 10^{5} \mathrm{RLU}$ after 24 hours to $8.3 \times 10^{6}$ and $5.9 \times 10^{7}$ RLU 72 and 96 hours post-transfection. Mutation D503A within an acidic patch between $\alpha$-helix 13 and 14 (JGN-9) did not influence replication either. GLuc 
activities reached $4 \times 10^{8}$ and $2.2 \times 10^{8} \mathrm{RLU} 72$ and 96 hours post-transfection. Furthermore, substitutions E508A and R512A (JGN-10) within $\alpha$-helix 14 were not implicated in replication. GLuc activity was $7.5 \times 10^{6} \mathrm{RLU}$ and $3 \times 10^{7} \mathrm{RLU}$ at the $72 \mathrm{~h}$ and 96 hour measurements. Constructs including upstream mutations T537A, T540A and H541A (JGN-12) showed ability to replicate. GLuc activity elevated from $3.9 \times 10^{4}$ RLU 24 hours post-transfection to $3.5 \times 10^{7}$ and $2.9 \times 10^{7}$ RLU 72 and 96 hours post-transfection. Constructs with mutations S548A and Q552A (JGN-15) within this $\alpha$-helix were also able to replicate. Values were $1.5 \times 10^{7}$ and $2.1 \times 10^{7} \mathrm{RLU}$ at both time points 72 and $96 \mathrm{~h}$.

Remaining three constructs within a-helix 14, 15 and 16 - JGN3-11, JGN3-14 and JGN3-14 - of NS3hel domain 3 revealed significantly low GLuc levels showing severe defects in replication. Within a-helix 15 mutation E530A combined with E533A (JGN-11) severely reduced replication. GLuc activity did not exceed $1.6 \times 10^{5} \mathrm{RLU}$. Time points $24 \mathrm{~h}, 72 \mathrm{~h}$ and 96 hours remained $3.5 \times 10^{4}, 5 \times 10^{4}$ and $3.5 \times 10^{4} \mathrm{RLU}$. Alanine substitutions of residues D543 and H545 (JGN-14) within a-helix 16 also reduced replication. Measured RLU levels did not rise over $1.2 \times 10^{5} \mathrm{RLU}$ at all time points. Combination of the four mutations D543A, H545A, S548A and Q552A within a-helix 16 (JGN-13) lead to severely reduced replication with GLuc levels less than $4.6 \times 10^{5} \mathrm{RLU}$.

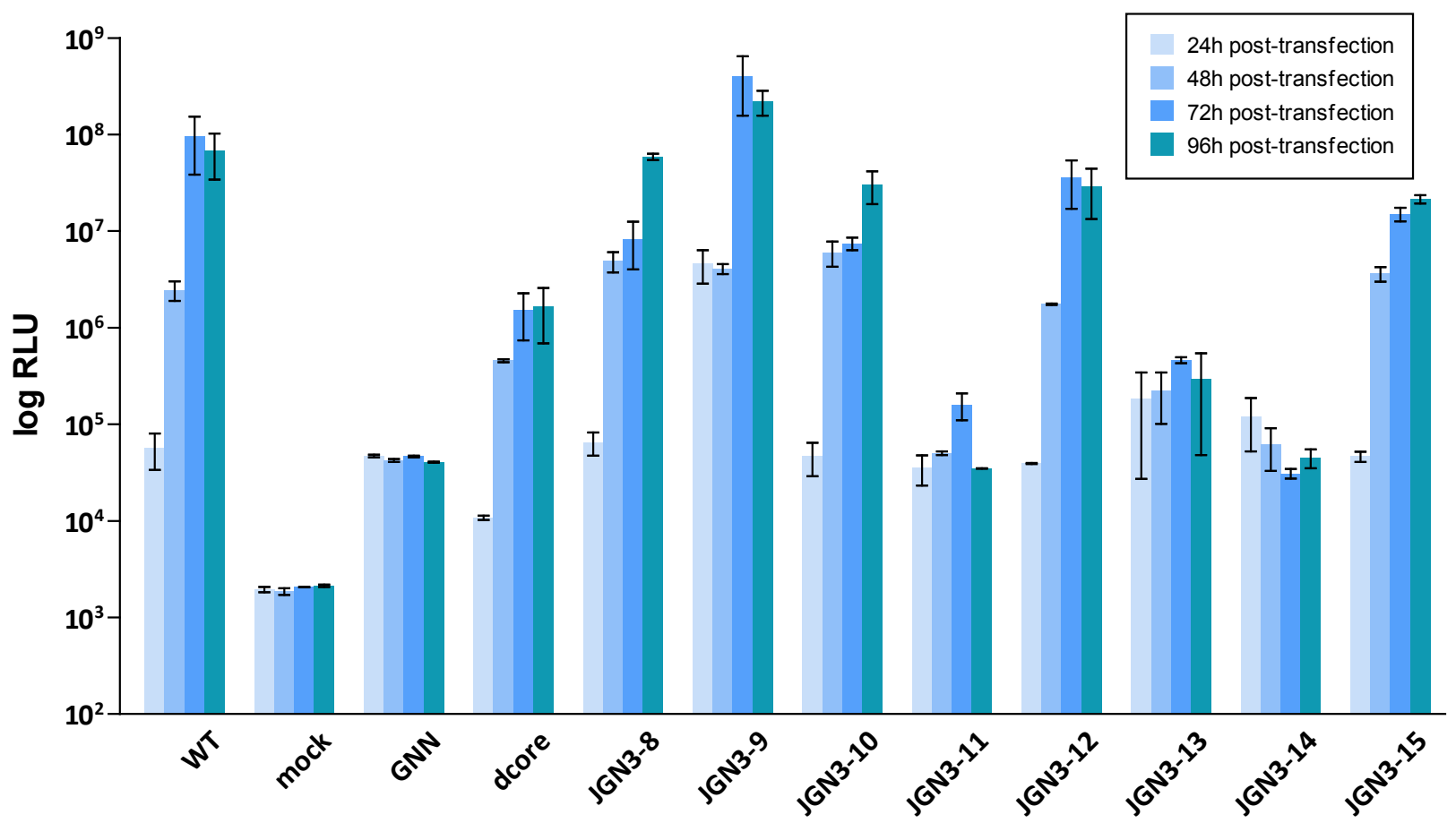

Figure 24 Replication of JGN3 constructs with changes in alpha helices 14, 15 and 16 of domain 3 . Cells were transfected with described mutants and controls. Medium was collected $24 \mathrm{~h}, 48 \mathrm{~h}, 72 \mathrm{~h}$ and $96 \mathrm{~h}$ post-transfection. Data represents secreted GLuc activity over time in Relative Light Units (RLU). Wild-type Jc1 (WT) is shown as a positive control. Further controls mock, GNN and $\Delta$ core (described above) are also shown. Values represent average measurements of at least two to three individual rounds of experiments. Error bars represent standard deviation of the mean.

Within NS3 helicase domain 3 a total of eight JGN3 mutants were able to replicate as efficient as WT. Four constructs showed severely defects in replication. The remaining three mutants showed reduced replication capacity. 
Results reveal that $\alpha$-helices $15,16,20$ and 21 play a role in viral replication. Whereas scanned residues within $\alpha$-helix 14 as well as the acidic path between $\alpha$-helices 13 and 14 do not seem to influence replication.

\subsubsection{Constructs with changes within NS3hel domain 2}

Within NS3hel domain 2 seven residues implicated in flavivirus assembly were substituted. Four of the changes are located within $\beta$ sheet E1. Remaining three mutations are adjacent to this structure.

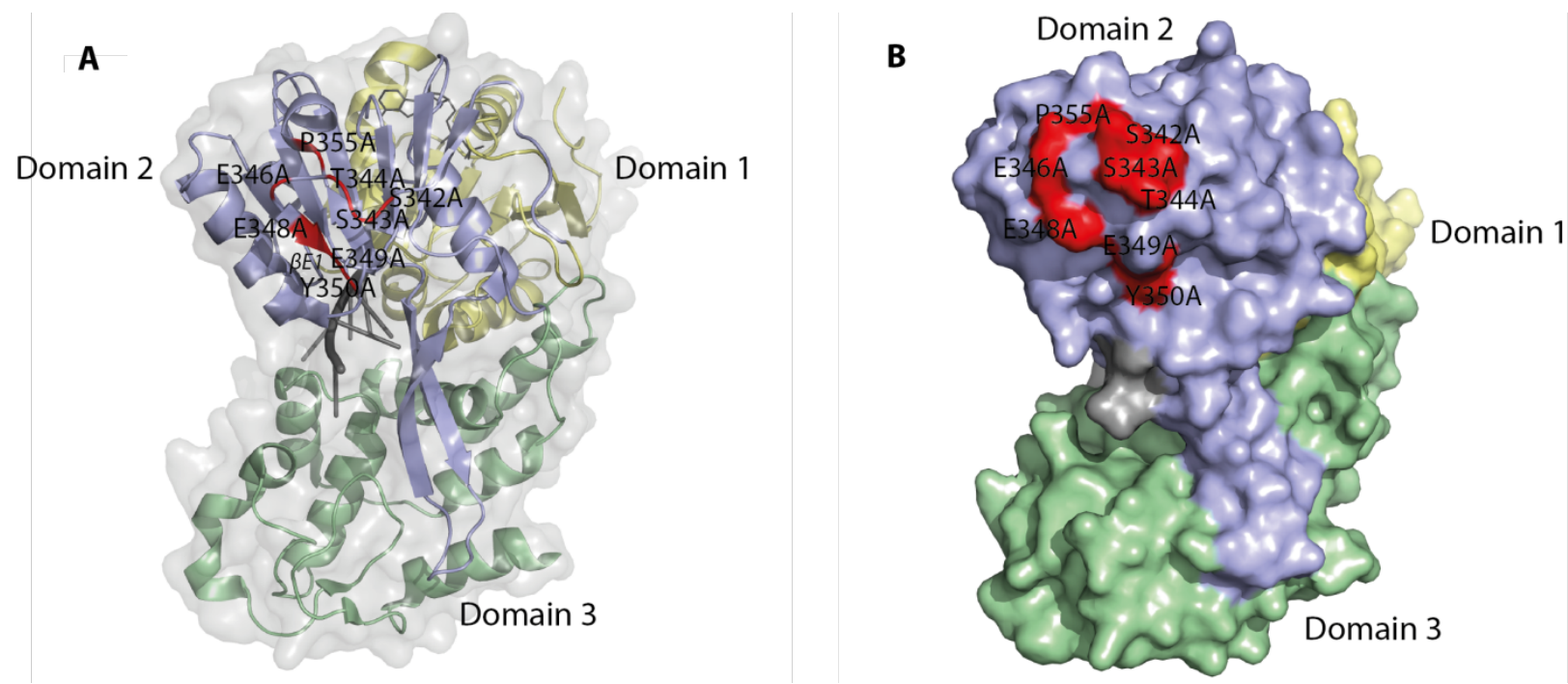

Figure 25 Mutation locations in NS3hel domain 2. (A) Ribbon model of NS3hel. Domain 1, domain 2 and domain 3 are colored yellow, lightblue and green. Substituted residues are highlighted red and marked by 1-letter-code residue name and position. (B) Surface model of NS3hel. Mutated residues are highlighted red. Models were rendered with PyMOL, Protein Data Bank code 3KQL.

\begin{tabular}{lll}
\hline Name of construct & Mutation & Location of Alanine Substitution \\
\hline JGN3-16 & E346A & Residues implicated in flavivirus \\
& P348A & assembly, \\
& F349A & within BE1 \\
& Y350A & \\
& P355A & \\
\hline JGN3-17 & E346A & \\
& P348A & \\
\hline JGN3-18 & F349A & \\
& P355A & Adjacent residues \\
\hline JGN3-19 & Y350A & \\
\hline JGN3-20 & S342A & \\
& S343A & T344A
\end{tabular}

Table 15 Changes within NS3hel domain 2. 
Replication was screened with the previously described system and mutants were compared to WT, mock, GNN and $\Delta$ core controls.

Within NS3 helicase domain 2 four JGN3 mutants - JGN3-16, JGN3-17, JGN3-18 and JGN3-19 - showed significantly low levels of GLuc activity compared to WT GLuc activity. All four constructs showing severe defects in viral replication had changes within NS3hel domain $2 \beta$-sheet E1. Construct JGN3-16 containing the combination of alanine substitutions E346A, P348A, F349A, Y350A and P355A showed a significantly reduced replication activity compared to the WT Jc1/GLuc2 phenotype. GLuc levels ranged between $5.1 \times 10^{4}$ and $2.5 \times 10^{5}$ RLU. 72 and 96 hours post-transfection GLuc activity values were measured about 1000 fold lower than the efficiently replicating WT. Separate mutations in the region of this beta sheet showed the same phenotype. Substitutions E346A and P348A (JGN3-17) as well as F349A and P355A (JGN3-18) revealed a defect in replication. JGN3-17 GLuc activities were 100 to 1000 fold lower than WT. Values around $1.5 \times 10^{5}$ RLU measured for JGN3-18 at 72 and 96 hours post-transfection also showed a replication defect. Furthermore, single mutation Y350A in JGN3-19 also lead to significant reduction of viral replication. This data implies that this region has to be critical for replication.

The remainder of mutants within domain 2 - JGN3-20 - showed a reduction of GLuc activity. Alanine substitutions of residues G342, R343 and E344 adjacent to beta sheet E1 did not reduce replication activity of JGN3-20. Measured values stagnated between $2.9 \times 10^{6}$ and $9.1 \times 10^{6} \mathrm{RLU}$ at 48 to 96 hour time points post-transfection. Results revealed that all scanned mutants with changes in NS3hel domain 2 influence viral replication. All of these constructs were located within beta sheet $\mathrm{E} 1$. Changes in adjacent residues reduced replication capacity. Data implicated that NS3 domain 2 is involved in viral replication as discussed later. 


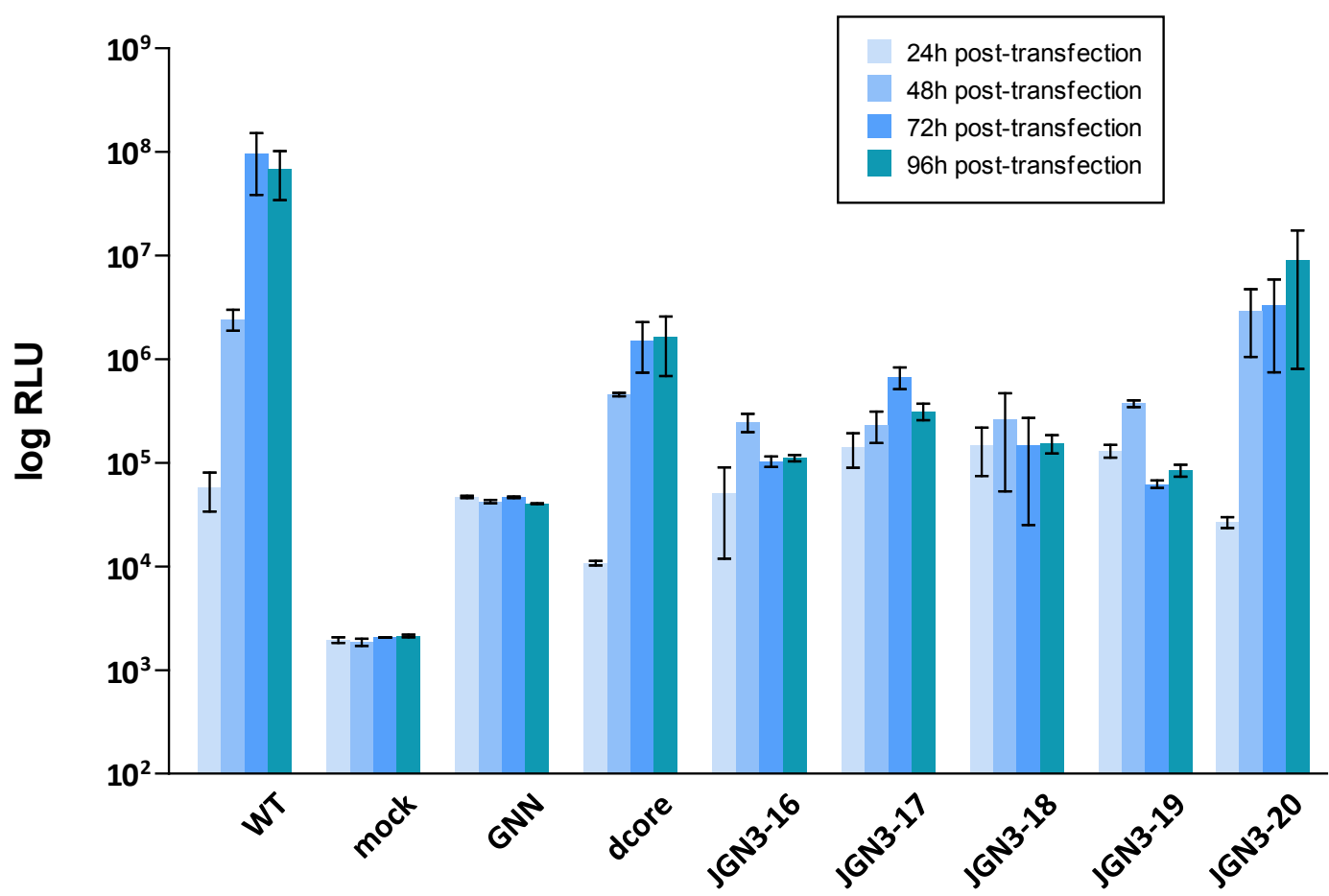

Figure 26 Replication of JGN3 constructs with changes in domain 2. Cells were transfected with described mutants and medium was collected $24 \mathrm{~h}, 48 \mathrm{~h}, 72 \mathrm{~h}$ and $96 \mathrm{~h}$ post-transfection. Data represents secreted GLuc activity over time. Wild-type Jc1 (WT) is shown as a positive control. Further controls mock, GNN and $\Delta$ core (described above) are also shown. Results represent at two to three individual rounds of experiments. Error bars represent standard deviation of the mean.

\subsubsection{Constructs with changes within NS3hel domain 1}

Within Domain 1 four amino acid substitutions were made in a conserved surface on the 'back' of domain 1, projecting from alpha helix 8 . Another four residues were changed in areas which were implicated in HCV assembly located within domain 1.

A

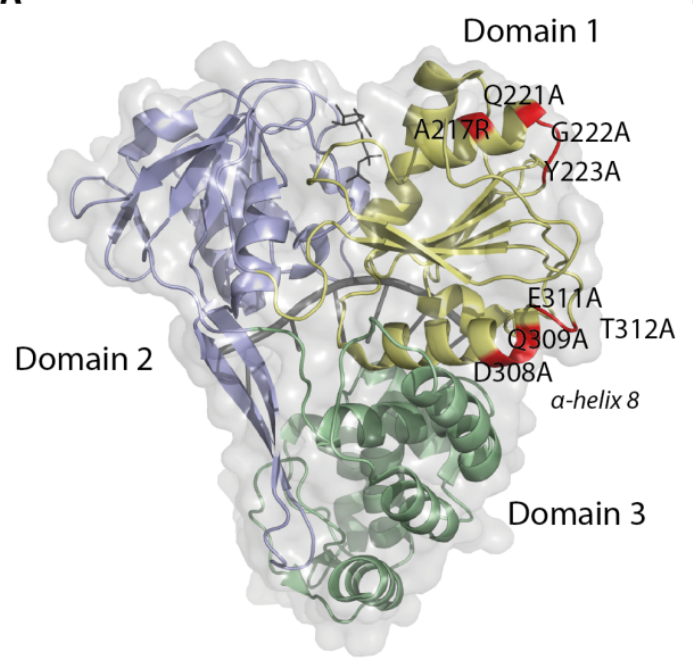

B

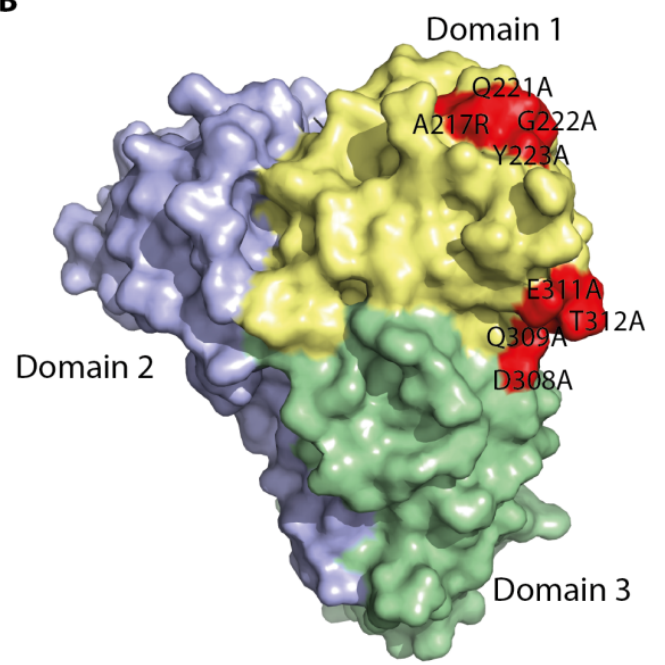

Figure 27 Mutation locations in NS3hel domain 2. (A) Ribbon model of NS3hel. Domain 1, domain 2 and domain 3 are colored yellow, lightblue and green. Substituted residues are highlighted red and marked by 1-letter-code residue name and position. (B) Surface model of NS3hel. Mutated residues are highlighted red. Models were rendered with PyMOL, Protein Data Bank code 3KQL. 


\begin{tabular}{lll}
\hline Name of construct & Mutation & Location of Alanine Substitution \\
\hline JGN3-21 & D308A & Conserved surface on back of domain 1, \\
& Q309A & Projecting from $\alpha$-helix 8 \\
& E311A & \\
& T312A & \\
\hline JGN3-22 & D308A & \\
& Q309A & \\
\hline JGN3-23 & E311A & Residues implicated in HCV assembly \\
& T312A & \\
\hline JGN3-24 & A217R & \\
\hline JGN3-25 & A217R & \\
& Q221A & \\
& G222A & \\
& Y223A & \\
\hline
\end{tabular}

Table 16 Changes within NS3hel domain 1.

Replication was screened with the previously described system and mutants were compared to WT, mock, GNN and $\Delta$ core controls as in the other domains.

Within NS3 helicase domain 1 four of the five studied mutants - JGN3-21, JGN3-22, JGN323 and JGN3-25 - were significantly influenced in replication. Changes of residues D308, Q309, E311A and D312A within a-helix 8 (JGN3-21) lead to reduced GLuc activities compared to WT. GLuc activities of $4.5 \times 10^{6}, 6.6 \times 10^{5}$ and $4.1 \times 10^{5} \mathrm{RLU}$ at 48,72 and 96 hours post-transfection were around 100 fold lower than WT. Combination of mutations D308A and Q309A as included in JGN3-22 also showed reduced GLuc activities. Values were $2.2 \times 10^{5}, 1.5 \times 10^{5}$ and $2.3 \times 10^{5} \mathrm{RLU}$ after 48,72 and 96 hour time points. Combination of mutations E311A and E312A, as scanned with JGN3-23 also significantly reduced GLuc activities. Values did not exceed $3.1 \times 10^{5} \mathrm{RLU}$ at all time points. Furthermore, combined mutations A217R, Q221A, G222A and Y223A (JGN3-25) severely reduced replication as well. GLuc activity was below $5.9 \times 10^{4} \mathrm{RLU}$ at all time points post-transfection.

Construct JGN3-24 which includes the A217R mutation was able to replicate efficiently. GLuc activity levels of $2.6 \times 10^{7}$ and $1.8 \times 10^{7} \mathrm{RLU} 72$ and 96 hours post-transfection were alike WT values.

Within NS3hel domain 1 all studied changes within alpha helix 8 lead to defects in replication as well as the mutant with changes around mutation Q221A. Data implicates that these regions are important for viral replication. One scanned mutant efficiently replicated similar to WT phenotype. 


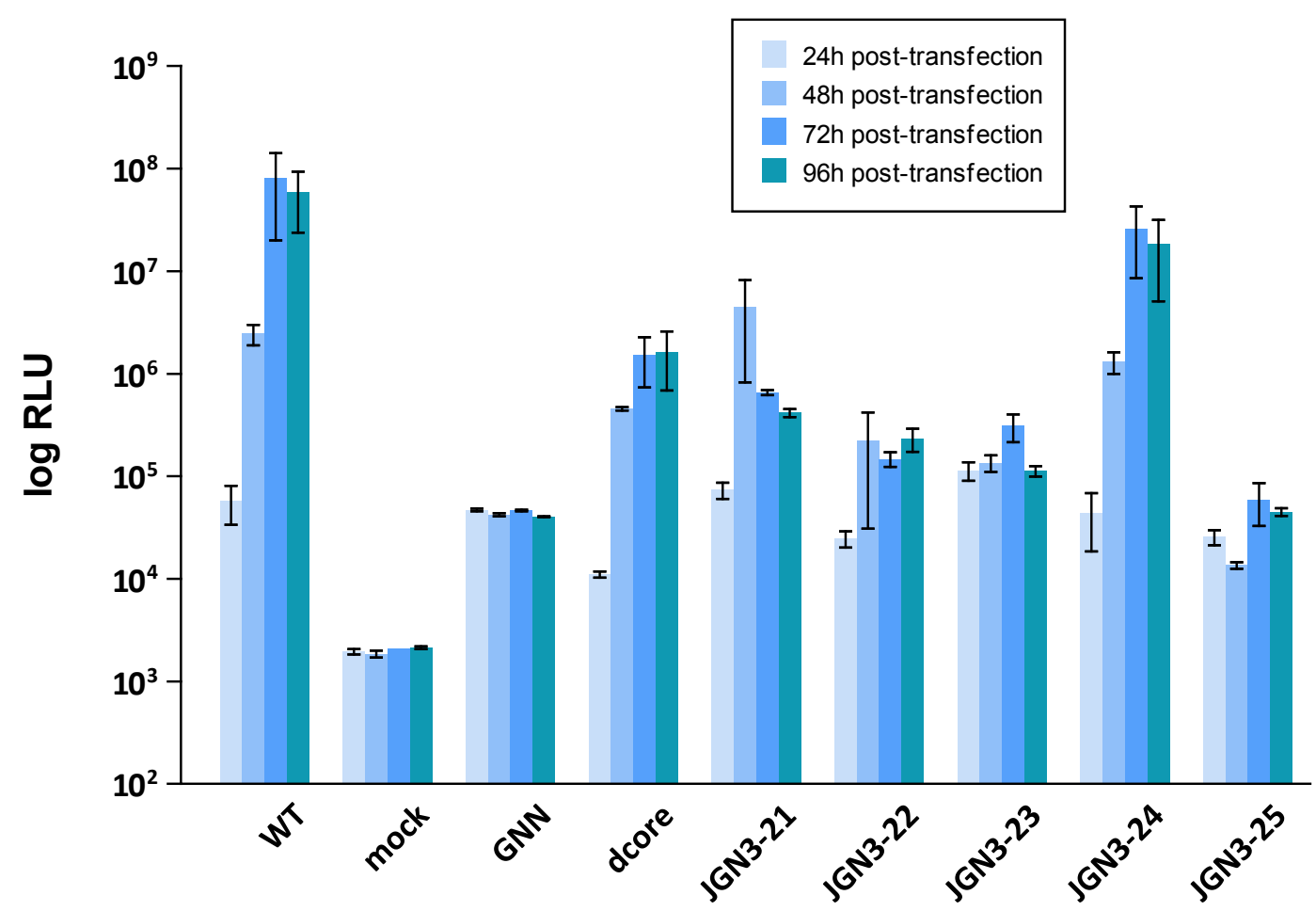

Figure 28 Replication of JGN3 constructs with changes in domain 1. Cells were transfected with described mutants and medium was collected $24 \mathrm{~h}, 48 \mathrm{~h}, 72 \mathrm{~h}$ and $96 \mathrm{~h}$ post-transfection. Data represents secreted GLuc activity over time. Wild-type Jc1 (WT) is shown as a positive control. Further controls mock, GNN and $\Delta$ core (described above) are also shown. Results represent at two to three individual rounds of experiments. Error bars represent standard deviation of the mean.

\subsubsection{Summary of replication results}

Replication experiments revealed some residues and some region within NS3hel domains which are important for viral replication. In each domain of NS3hel, sites that influence replication could be determined. Regions within domain 1 and domain 2 were especially sensitive for changes. Out of 25 scanned constructs 9 replicated similar to WT. 12 constructs had severe defects in replication. The remainder of mutants showed reduced GLuc activity. Within domain 2 beta sheet E1 was shown to play a role in viral replication. Alpha helix 8 of domain 1 was also sensitive for changes. Within domain 3 alpha helices 15, 16, 20 and 21 showed to be involved in HCV replication.

\subsection{Infectivity}

Mutants were also screened for infectivity. All constructs were monitored by infecting naïve Huh 7.5 cells with conditioned cell culture medium from the transfected cells collected at various time points. Following a $24 \mathrm{~h}$ absorption period cells were washed and harvested in fresh medium. Secreted GLuc was measured $72 \mathrm{~h}$ post-infection. WT was used as positive infectious control, while polymerase defective control GNN and mock were used for negative 
non-infectious controls. Jc1/GLuc2( $\Delta$ core) containing a deletion of the core gene was used as a control which is able to replicate but does not produce infectious virus particles. Constructs with severe defects in replication are expected not to be infectious as they are unable to replicate.

\subsubsection{Constructs with changes within NS3hel domain 3}

From 15 constructs screened with changes in domain 3, four did not replicate (JGN3-1, JGN3-11, JGN3-13 and JGN3-14) and another three showed reduced replication (JGN3-4, JGN3-6 and JGN3-7). Remaining eight mutants replicated efficiently.

As expected here WT Jc1/GLuc2 produced infectious virus. Measured GLuc activities rose from $1.9 \times 10^{4} \mathrm{RLU}$ when infected with supernatants harvested 24 hours post-transfection to $7.4 \times 10^{6}, 3.2 \times 10^{7}$ and $2 \times 10^{7} \mathrm{RLU}$ when infected with supernatants 48,72 and 96 hours posttransfection. Controls mock and GNN which did not replicate, were not infectious. GLuc activities were below $3 \times 10^{3} \mathrm{RLU}$. Jc1/GLuc2( $\Delta$ core) with a deletion of the core gene which was able to replicate, showed GLuc activities below $3.1 \times 10^{3} \mathrm{RLU}$ at all scanned time points.

\subsubsection{Infectivity of constructs with changes within NS3hel domain 3 alpha helix 20 and 21}

Within alpha helix 20 and 21 of NS3 helicase domain 3 constructs JGN3-2, JGN3-3 and JGN3-5 were able to replicate efficiently.

JGN3-3 showed ability to infect naive Huh7.5 cells alike WT. GLuc activity levels reached $4 \times 10^{6}$ and $1.4 \times 10^{7}$ RLU when infected with supernatants harvested 72 and 96 hours posttransfection. JGN3-5 showed GLuc activity levels of $1.3 \times 107$ and $1.7 \times 107$ RLU using supernatant collected 72 and 96 hours post-transfection.

Measured GLuc activities of mutant JGN3-2 were significantly lower than WT levels. GLuc activities stagnated between $1.9 \times 10^{4}$ and $2.4 \times 10^{4} \mathrm{RLU}$ when infected with supernatants harvested 48, 72 and 96 hours post-transfection which is around 10000 fold lower than WT GLuc activity.

Within alpha helix 20 and 21 of NS3hel domain 3 the construct that showed severe defects in replication (JGN3-1) showed significantly lower GLuc levels than WT. Values ranged between $2 \times 10^{3}$ and $1 \times 10^{4} \mathrm{RLU}$ during all time points used to infect naïve Huh7.5 cells. Scanned mutants JGN3-4, JGN3-6 and JGN3-7 that showed reduced GLuc levels during replication experiments revealed significantly lower GLuc activity levels during infectivity

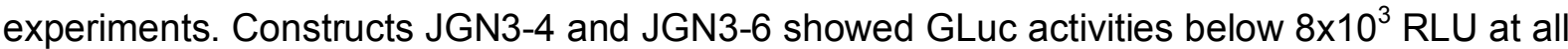
measured time points. Levels were 1000 to 10000 fold lower than WT levels. Construct JGN3-7 showed reduced GLuc activities during infectivity experiments. Values were between 
$5.7 \times 10^{4}$ and $1.2 \times 10^{5} \mathrm{RLU}$ using supernatant collected 72 and 96 hours post-transfection. Values were 10 fold higher compared to JGN3-4 and JGN3-6 and 100 fold lower than WT GLuc activities.

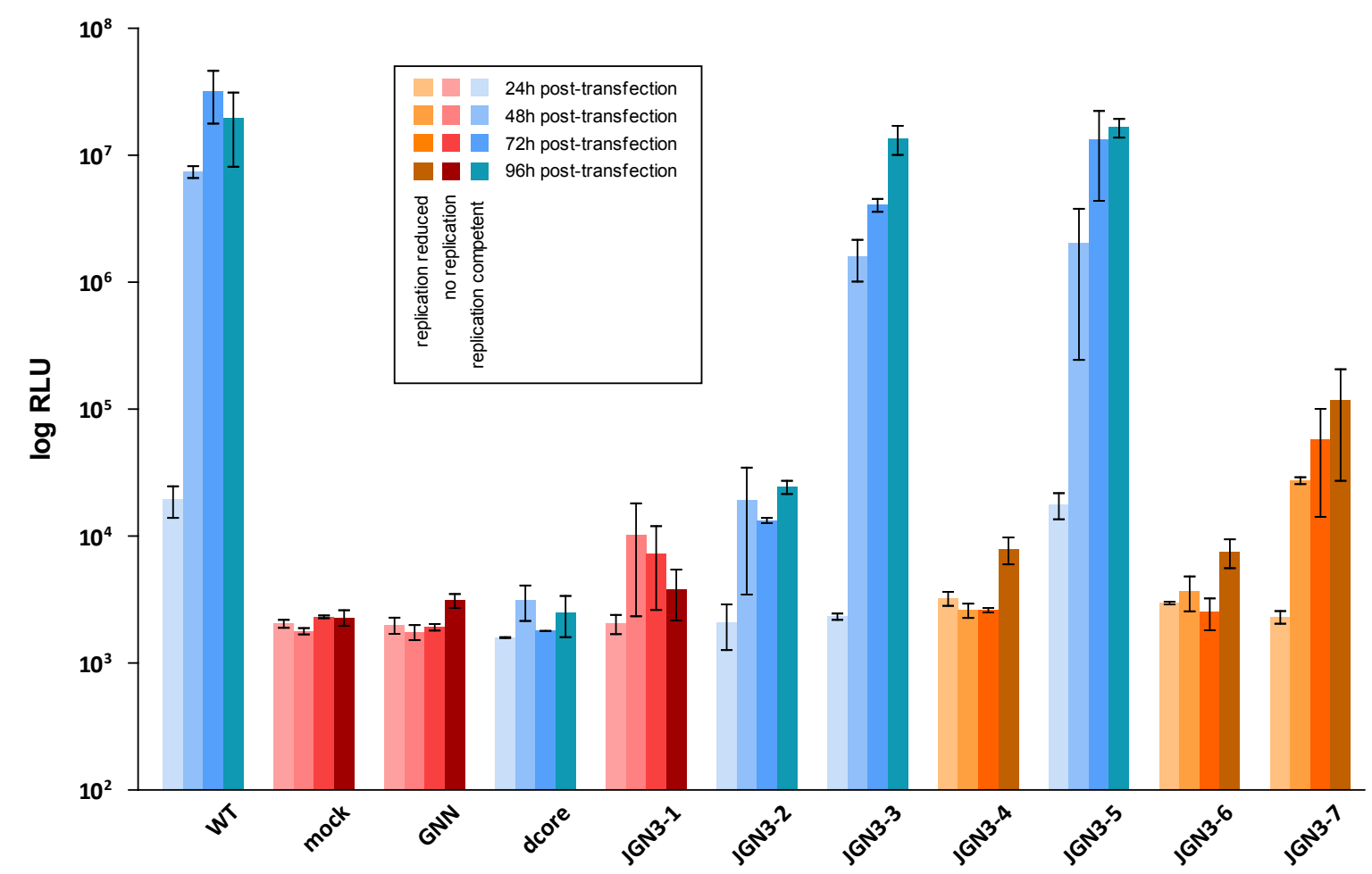

Figure 29 Infectivity results of JGN3 constructs with changes within domain $\mathbf{3}$ alpha helix $\mathbf{2 0}$ and $\mathbf{2 1}$. Cells were infected with collected medium ( $24 \mathrm{~h}, 48 \mathrm{~h}, 72 \mathrm{~h}$ and $96 \mathrm{~h}$ post-transfection) from transfection experiments. Cells were washes after $24 \mathrm{~h}$ and incubated in fresh medium for another $48 \mathrm{~h}$. Data represents secreted GLuc activity over time in relative light units (RLU). Wild-type Jc1 (WT) is shown as a positive control. Further controls mock, GNN and $\Delta$ core (described above) are also shown. Constructs with severe defects in replication are highlighted red. Constructs with reduced replication are highlighted orange. Results represent at least two to three individual rounds of experiments. Error bars indicate standard deviation of the mean.

\subsubsection{Infectivity of constructs with changes within NS3hel domain 3 alpha helix 14, 15 and} 16

Within alpha helix 14, 15 and 16 of NS3hel domain 3 five constructs - JGN3-8, JGN3-9, JGN3-10, JGN3-12 and JGN3-15 - showed ability to replicate efficiently. JGN3-9 showed GLuc activities of $9 \times 10^{6}$ and $1.1 \times 10^{7} \mathrm{RLU}$ using 72 and 96 hour supernatant to infect naïve cells. Both JGN3-8 and JGN3-10 showed decreased GLuc activity levels compared to WT. JGN3-8 revealed GLuc activities of $1.3 \times 10^{5}$ and $3.5 \times 10^{5} \mathrm{RLU}$ at 72 and 96 hour time points used for infections. JGN3-10 showed comparable results. Values were $3.8 \times 10^{5}$ and $4.4 \times 10^{5}$ RLU using 72 and 96 hour supernatants. Values of both constructs were around 100 fold lower than WT values. JGN3-15 showed slightly decreased GLuc activities of about 10 fold lower than WT levels. Infecting cells with supernatant harvested 72 and 96 hours posttransfection showed GLuc activities of $1.3 \times 10^{6}$ and $2 \times 10^{6} \mathrm{RLU}$. 
GLuc activity values of construct JGN3-12, that was able to replicate, were below $2 \times 10^{4} \mathrm{RLU}$ at all scanned time points. Values were significantly (about 10000 fold) lower than WT GLuc activities.

Remaining three mutants with changes within alpha helix 15 or 16 - JGN3-11, JGN3-13 and JGN3-14 - were not able to replicate. All three constructs showed around 10000 fold lower GLuc activities compared to WT values. JGN3-11 ranged around $2 \times 10^{3} \mathrm{RLU}$ during all scanned time points. JGN3-13 showed amounts lower than $3 \times 10^{3}$ RLU. GLuc activities of JGN3-14 were between $2 \times 10^{3}$ and $4.7 \times 10^{3}$ using supernatant harvested 24 to 96 hours posttransfection.

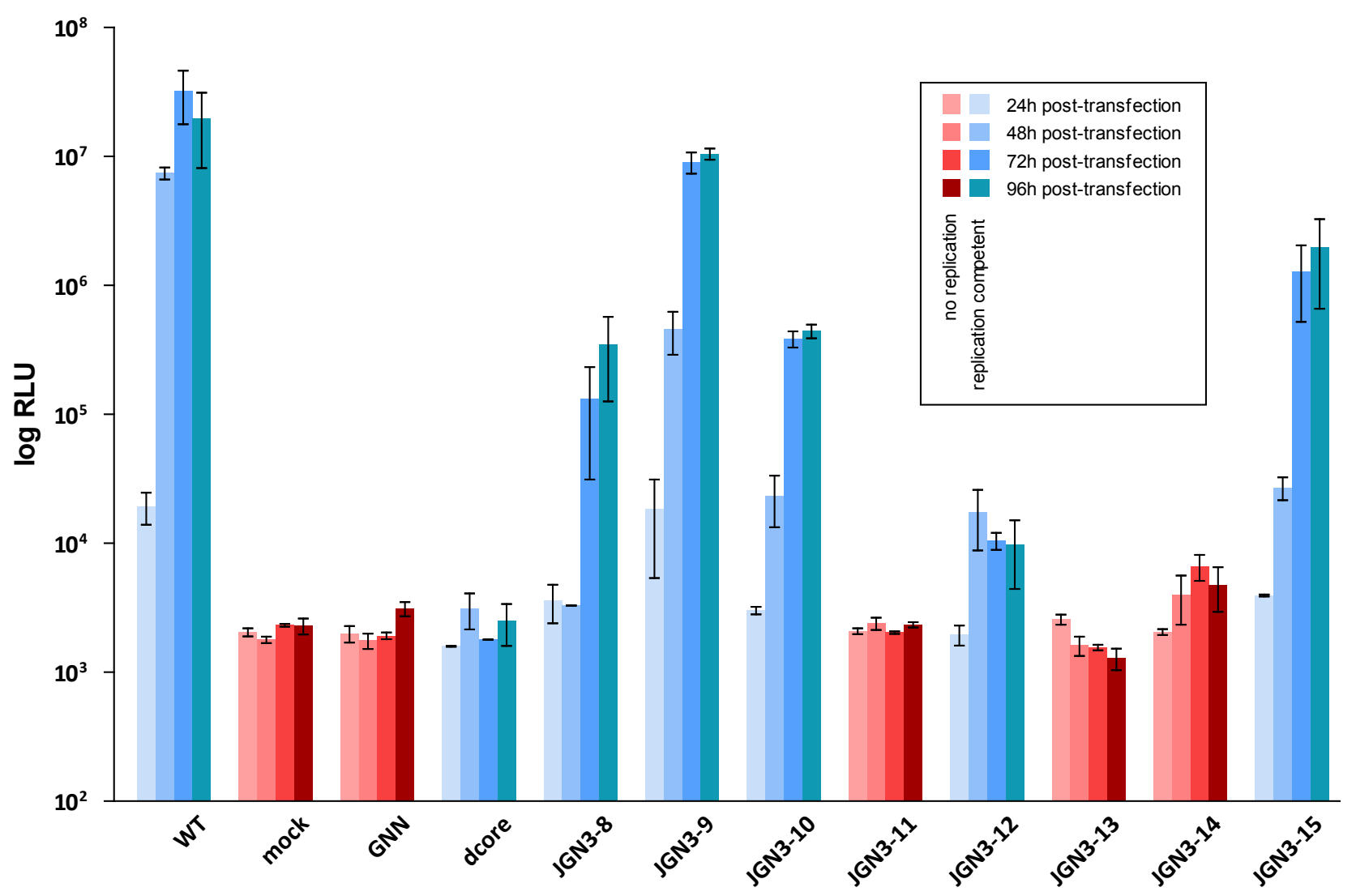

Figure 30 Infectivity of JGN3 constructs with changes within domain 3 alpha helix 14, 15 and 16 . Cells were infected with collected medium ( $24 \mathrm{~h}, 48 \mathrm{~h}, 72 \mathrm{~h}$ and $96 \mathrm{~h}$ post-transfection) from transfection experiments. Cells were washes after $24 \mathrm{~h}$ and incubated in fresh medium for another $48 \mathrm{~h}$. Data represents secreted GLuc activity over time. Wild-type Jc1 (WT) is shown as a positive control. Further controls mock, GNN and $\Delta$ core (described above) are also shown. Constructs with severe defects in replication are highlighted red. Constructs with reduced replication are highlighted orange. Results represent at least two to three individual rounds of experiments. Error bars indicate standard deviation of the mean.

\subsubsection{Constructs with changes within NS3hel domain 2}

All five screened mutations within NS3 helicase domain 2 (JGN3-16, JGN3-17, JGN3-18, JGN3-19, JGN3-20) caused severe or moderate defects in replication. All of these constructs showed around 1000 to 10000 fold lower GLuc activity levels than WT. 
Within infectivity experiments using supernatants $24,48,72$ and 96 hours post-transfection, GLuc values of JGN3-16 ranged from $1.8 \times 10^{3}$ to $6 \times 10^{4}$ RLU. JGN3-17 GLuc activity values stagnated around $3 \times 10^{3}$ at all tested time points. Furthermore, both JGN3-18 and JGN3-19 revealed GLuc activity levels under $2 \times 10^{3} \mathrm{RLU}$ at all time points scanned for infection. JGN320 showed reduced GLuc activities compared to WT with values between $2.7 \times 10^{3}$ and $5 \times 10^{4}$ RLU infecting naïve cells with supernatant harvested 24 to 96 hours post-transfection.

Results reveal that none of the constructs with changes in domain 2 were able to infect naïve Huh7.5 cells.

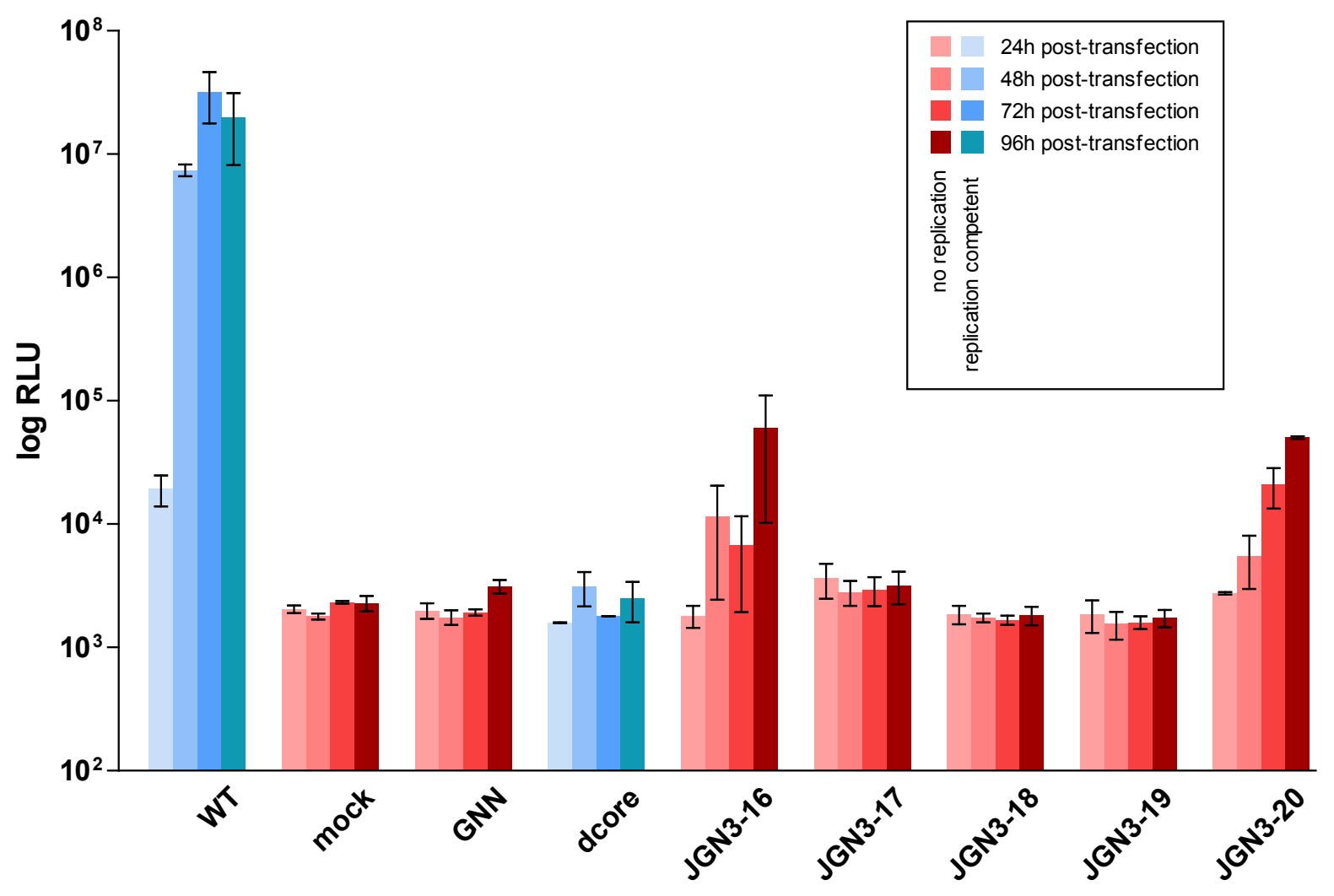

Figure 31 Infectivity of JGN3 constructs with changes within domain 2. Cells were infected with collected medium (24h, $48 \mathrm{~h}, 72 \mathrm{~h}$ and $96 \mathrm{~h}$ post-transfection) from transfection experiments. Cells were washes after $24 \mathrm{~h}$ and incubated in fresh medium for another $48 \mathrm{~h}$. Data represents secreted GLuc activity over time. Wild-type Jc1 (WT) is shown as a positive control. Further controls mock, GNN and $\Delta$ core (described above) are also shown. Constructs with severe defects in replication are highlighted red. Results represent two to three individual rounds of experiments. Error bars represent standard deviation of the mean.

\subsubsection{Constructs with changes within NS3hel domain 1}

Within NS3hel domain 1 only construct JGN3-24, containing the A217R mutation, showed efficient replication. The other changes within domain 1 lead to severe or moderate defects in replication.

Replication defective mutants JGN3-21, JGN3-22, JGN3-23 and JGN3-25 showed significantly reduced GLuc activity levels compared to WT. Values were 100000 fold lower 
than WT values. JGN3-21, JGN3-22 and JGN3-23 showed GLuc activity below $3 \times 10^{3}$ RLU. JGN3-25 results ranged between $5 \times 10^{3}$ and $2.6 \times 10^{3} \mathrm{RLU}$.

Replication competent mutant JGN3-24 also showed significantly lower GLuc levels than WT. Values were between $1.6 \times 10^{3}$ and $2 \times 10^{4} \mathrm{RLU}$, showing that JGN3-24 is able to replicate but not to infect naïve Huh7.5 cells.

Results showed that none of the examined constructs with changes in domain 1 were infectious, not even JGN3-24 which replicated efficiently.

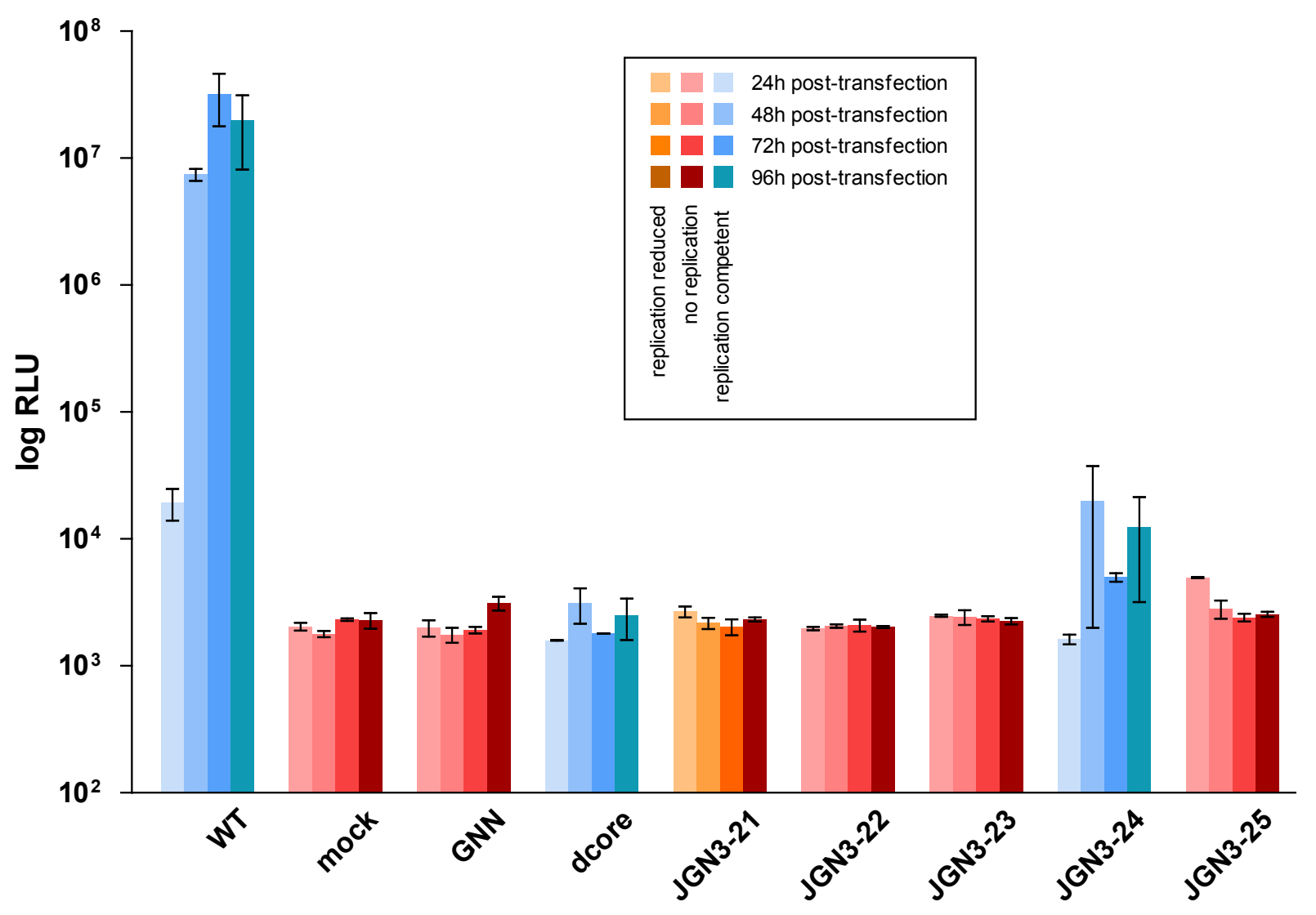

Figure 32 Infectivity of JGN3 constructs with changes within domain 1. Cells were infected with collected medium (24h, $48 \mathrm{~h}, 72 \mathrm{~h}$ and $96 \mathrm{~h}$ post-transfection) from transfection experiments. Cells were washes after $24 \mathrm{~h}$ and incubated in fresh medium for another $48 \mathrm{~h}$. Data represents secreted GLuc activity over time. Wild-type Jc1 (WT) is shown as a positive control. Further controls mock, GNN and $\Delta$ core (described above) are also shown. Constructs with severe defects in replication are highlighted red. Constructs with reduced replication are highlighted orange. Results represent at least two to three individual rounds of experiments. Error bars represent standard deviation of the mean.

\subsubsection{Summary of infectivity experiments}

15 studied constructs that were shown to have severe or moderate defects in replication JGN3-1, JGN3-4, JGN3-6, JGN3-11, JGN3-13, JGN3-14, JGN3-16, JGN3-17, JGN3-18, JGN3-19, JGN3-20, JGN3-21, JGN3-22, JGN3-23 and JGN3-25 - were shown to be noninfectious. Construct JGN3-7 that showed reduced replication also showed reduced GLuc levels within infectivity experiments but seems to be infectious. 
Out of nine mutants that were shown to be replication competent six constructs - JGN3-3, JGN3-5, JGN3-8, JGN3-9, JGN3-10 and JGN3-15 - were able to infect naïve Huh7.5 cells as efficient or almost as efficient as WT. Most interestingly, three mutants were able to replicate but were not infectious. These mutants include two constructs with changes in domain 3: JGN3-2 with substitutions T612A, H613A within a-helix 21 and JGN3-12 with changes T537A, T540A and H541A within a-helix 15 of domain 3. One construct showing these properties could be determined within domain 1: JGN3-24 that includes mutation A217R within $\alpha$-helix 5. Due to these findings these residues are candidates to be involved in virion assembly. These three mutants were used for further studies and were the center of this thesis.

\subsection{Anti-NS5a staining}

Replication and infectivity results were confirmed by immunostaining for NS5a. Huh 7.5 cells were transfected, fixed and stained $72 \mathrm{~h}$ post-transfection. Successfully transfected cells that provide efficient HCV replication can be positively stained for NS5a as well as infected cells with efficient HCV replication. Cells were fixated, incubated with an anti-NS5a monoclonal antibody and stained. Cells that include a certain amount of NS5a can be detected. Results of immunostaining confirmed data of HCV replication and infectivity results.

Constructs that were shown to replicate due to its GLuc activity levels comparable to WT could be positively stained for NS5A. Within mutants that revealed defects in replication with significantly low GLuc activity levels, NS5A could not be detected with immunostaining. Constructs JGN3-4, JGN3-6, JGN3-20 and JGN3-21 that had reduced GLuc activities within replication experiments were stained negatively for NS5A. However, JGN3-7 that also revealed reduced GLuc activities could be positively stained for NS5A.

Furthermore, these experiments were able to confirm the mutant's competence of infectivity. Constructs with GLuc levels alike WT within infectivity experiments were confirmed to be as infectious as WT. NS5A could be positively stained in these cells. Staining results of constructs with severely low GLuc activities were negative. Cells infected with supernatant from JGN3-7, JGN3-8 and JGN3-10, that showed around 10 fold lower GLuc activities compared to WT, could be positively stained and shown to be infectious within these experiments. Details of staining results are shown in figure 33 . 


\begin{tabular}{|c|c|c|c|c|}
\hline $\begin{array}{l}\text { Name of } \\
\text { construct }\end{array}$ & $\begin{array}{l}\text { GLuc activity } \\
\text { replication } \\
\text { experiments }\end{array}$ & $\begin{array}{l}\text { GLuc activity } \\
\text { infectivity } \\
\text { experiments }\end{array}$ & $\begin{array}{c}\text { Staining } \\
\text { replication } \\
\text { experiments }\end{array}$ & $\begin{array}{c}\text { Staining } \\
\text { infectivity } \\
\text { experiments }\end{array}$ \\
\hline WT & + & + & $0_{0}^{0} 0$ & \\
\hline GNN & - & - & (0) 0 & (0) 0 \\
\hline$\Delta$ core & + & - & $0^{\circ} 0$ & (0) \\
\hline JGN3-1 & - & - & (0) 0 & (0) 0 \\
\hline JGN3-2 & + & - & (0) & (0) \\
\hline JGN3-3 & + & + & $0_{0}^{0} 0$ & 0 \\
\hline JGN3-4 & reduced & - & (0) 0 & 8 \\
\hline JGN3-5 & + & + & $0_{0}^{0} 0$ & 0 \\
\hline JGN3-6 & reduced & - & 0 (0) & 0 (0) \\
\hline JGN3-7 & reduced & reduced & $0^{\circ} 0$ & 0 \\
\hline JGN3-8 & + & reduced & $0^{0} 0$ & $0_{0}^{0} 0$ \\
\hline JGN3-9 & + & + & $0_{0}^{0} 0$ & $0_{0}^{0} 0$ \\
\hline JGN3-10 & + & reduced & $0_{0}^{0} 0$ & 0 \\
\hline JGN3-11 & - & - & 0 (0) 0 & 0 (0) \\
\hline JGN3-12 & + & - & $0_{0}^{0} 0$ & 0)(0) \\
\hline JGN3-13 & - & - & (0) 0 & (0) 0 \\
\hline JGN3-14 & - & - & (0) 0 & (0) 0 \\
\hline JGN3-15 & + & + & $0^{0} 0$ & $0_{0}^{0} 0$ \\
\hline JGN3-16 & - & - & (0) 0 & (0) 0 \\
\hline JGN3-17 & - & - & 0 (0) 0 & (0) 0 \\
\hline JGN3-18 & - & - & (0) 0 & (0) \\
\hline JGN3-19 & - & - & 0 (0) 0 & 0 (0) 0 \\
\hline JGN3-20 & reduced & - & 0 (0) 0 & 0 (0) \\
\hline JGN3-21 & reduced & - & (0) 0 & (0) 0 \\
\hline JGN3-22 & - & - & 0 (0) 0 & 0 (0) 0 \\
\hline
\end{tabular}




\begin{tabular}{|lcccr|}
\hline $\begin{array}{l}\text { Name of } \\
\text { construct }\end{array}$ & $\begin{array}{c}\text { GLuc activity } \\
\text { replication } \\
\text { experiments }\end{array}$ & $\begin{array}{c}\text { GLuc activity } \\
\text { infectivity } \\
\text { experiments }\end{array}$ & $\begin{array}{c}\text { Staining } \\
\text { replication } \\
\text { experiments }\end{array}$ & $\begin{array}{c}\text { Staining } \\
\text { infectivity } \\
\text { experiments }\end{array}$ \\
\hline JGN3-23 & - & - & - & 0 \\
\hline JGN3-24 & + & - & 0 & 0 \\
\hline JGN3-25 & - & - & 0 & 0 \\
\hline
\end{tabular}

Figure 33 Summary of replication and infectivity experiments and NS5a immunostaining results. Efficient replication and infectivity in the GLuc experiments are marked by +, defects in replication or infectivity with - and reduced replication by $r$. Results of staining experiments are represented by colored cell models; blue cells represent unstained cells and brown cells represent positive staining of NS5a. Results represent at least two to three individual rounds of experiments.

\subsection{Studying HCV virus particle assembly: JGN3-2, JGN3-12 and JGN3-24 - constructs that were able to replicate but not to be infectious}

Three of 25 screened constructs showed the ability to replicate but did not infect naïve Huh 7.5 cells. These constructs that contain mutations within $\alpha$-helix 21 and $\alpha$-helix 15 in domain 3 and within a-helix 5 of domain 1 are interesting for studying the production of infectious HCV particles.

The shown phenotypes predestinate the mutants to serve as models to study the still unknown process of HCV assembly. Some questions have to be answered to determine the lack of infectivity of these mutants. It has to be examined if the problem can be found within the process of virus particle release or assembly of viral particles. Furthermore, it has to be determined if intracellular infectious viral particles can be formed or if these mutations lead to a defect in an earlier step of virion assembly. Constructs were also passaged at cell culture experiments for an extended period of time studying the effect of long term incubation and changes of this highly mutating virus.

Another question was, whether these mutations influence the formation of a newly described complex with NS2 and the viral glycoproteins or if they influence viral assembly and infectivity in another way.

Characteristics of JGN3-2, JGN3-12 and JGN3-24 and results experiments described above are pooled in figure 34 . 
A

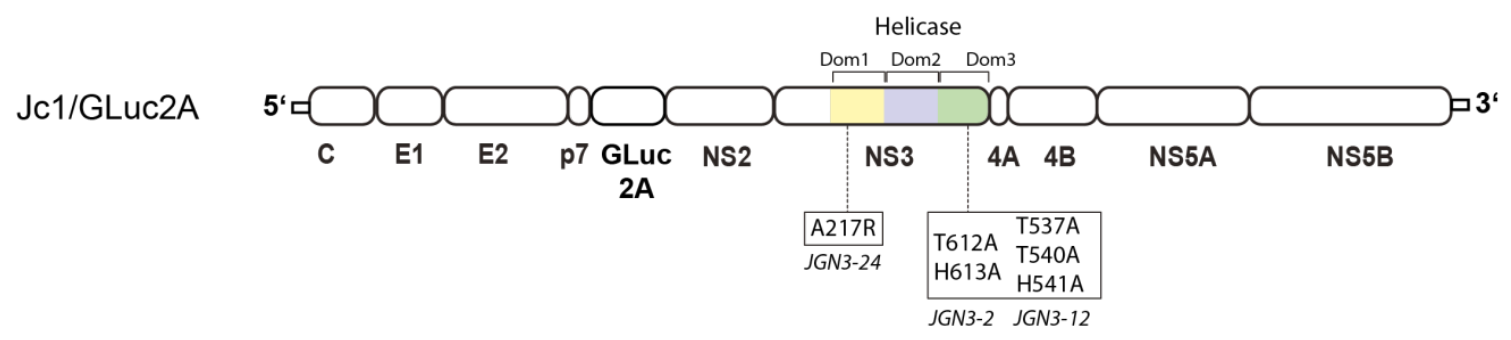

B

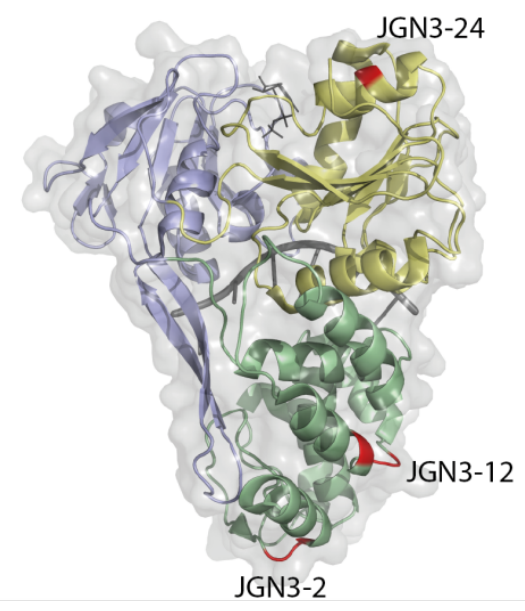

C

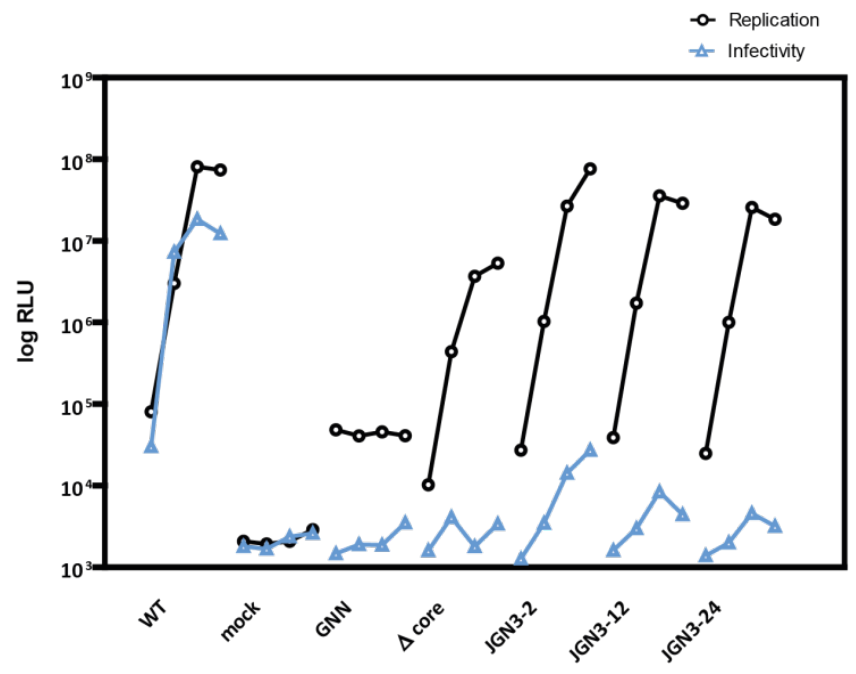

$\Delta$ core

JGN3-2

JGN3-12

JGN3-24
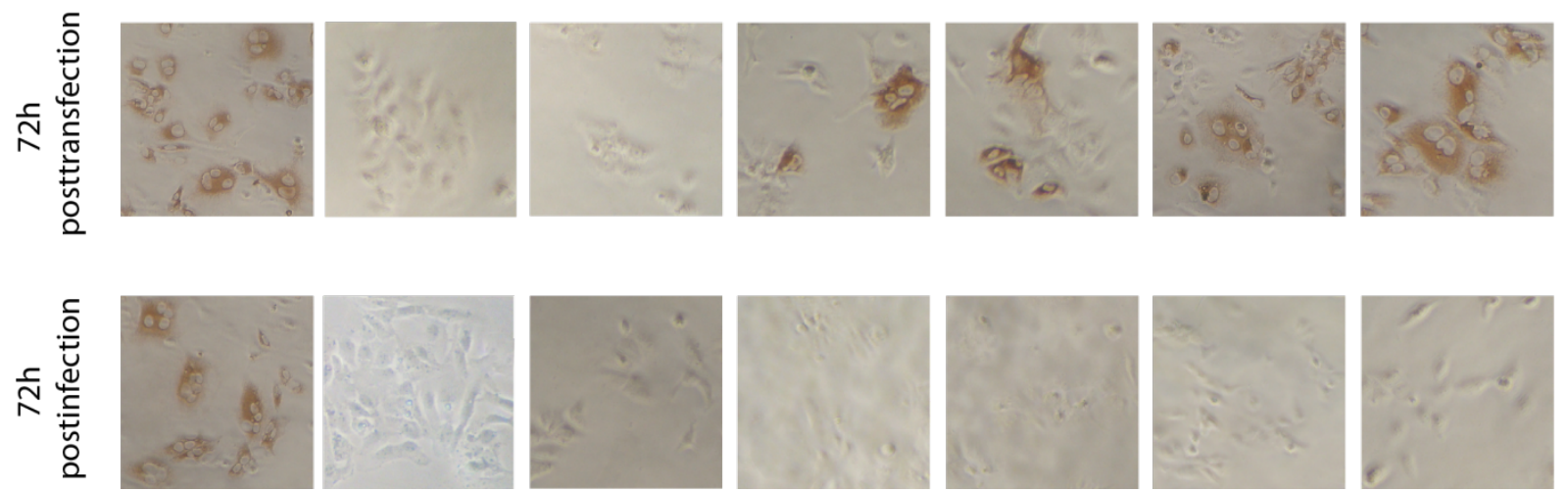

Figure 34 JGN3-2, JGN3-12 and JGN3-24 - mutations, replication and infectivity. (A) Jc1/GLuc2 construct used in these studies. NS3hel domains are colored yellow, lightblue and green. Mutations within JGN3-2 (T612A, H613A), JGN3-12 (T537A, T540A, H541A) and JGN3-24 (A217R) are shown. (B) Ribbon structure of NS3hel. Domains are colored yellow, lightblue and green according to coloring above. Mutation sites are highlighted red. (C) Summary of replication and infectivity data of JGN3-2, JGN3-12 and JGN3-24. Curves represent GLuc activity in relative light units (RLU). Black curves show replication $24 \mathrm{~h}, 48 \mathrm{~h}, 72 \mathrm{~h}$ and $96 \mathrm{~h}$ post-transfection. Blue curves show infectivity of native Huh 7.5 cells infected with medium collected in transfection experiments at mentioned time points. (D) NS5a immunostaining of Huh 7.5 cells posttransfection and post-infection. Staining confirms results of GLuc reporter virus experiments. WT, $\Delta$ core, JGN3-2, JGN3-12 and JGN3-24 replicate in Huh 7.5 cells. NS5a is detected by anti-NS5a staining (as described in 'Material and Methods' p.40) within the cells. Just WT Jc1/GLuc2 is able to infect native Huh 7.5 cells. NS5a cannot be detected within the remainder of infection experiments. Results represent at least three individual rounds of experiments. 


\subsubsection{Release of viral particles}

As described above mutants that were able to replicate but did not show to be infectious (JGN3-2, JGN3-12 and JGN3-24) were studied closer. Different reasons could cause the defect in infectivity of these mutants. The inserted mutations could inhibit the release of viral particles. Another theoretical explanation could be that viral particles are released but not infectious. To determine between these two possibilities the amount of viral RNA within the cell culture medium was measured by quantitative RT-PCR as the amount of HCV RNA within the cell culture medium represents released viral particles. Huh 7.5 cells were transfected and medium was used to measure the amount of RNA present in the medium at 6 days post-transfection.

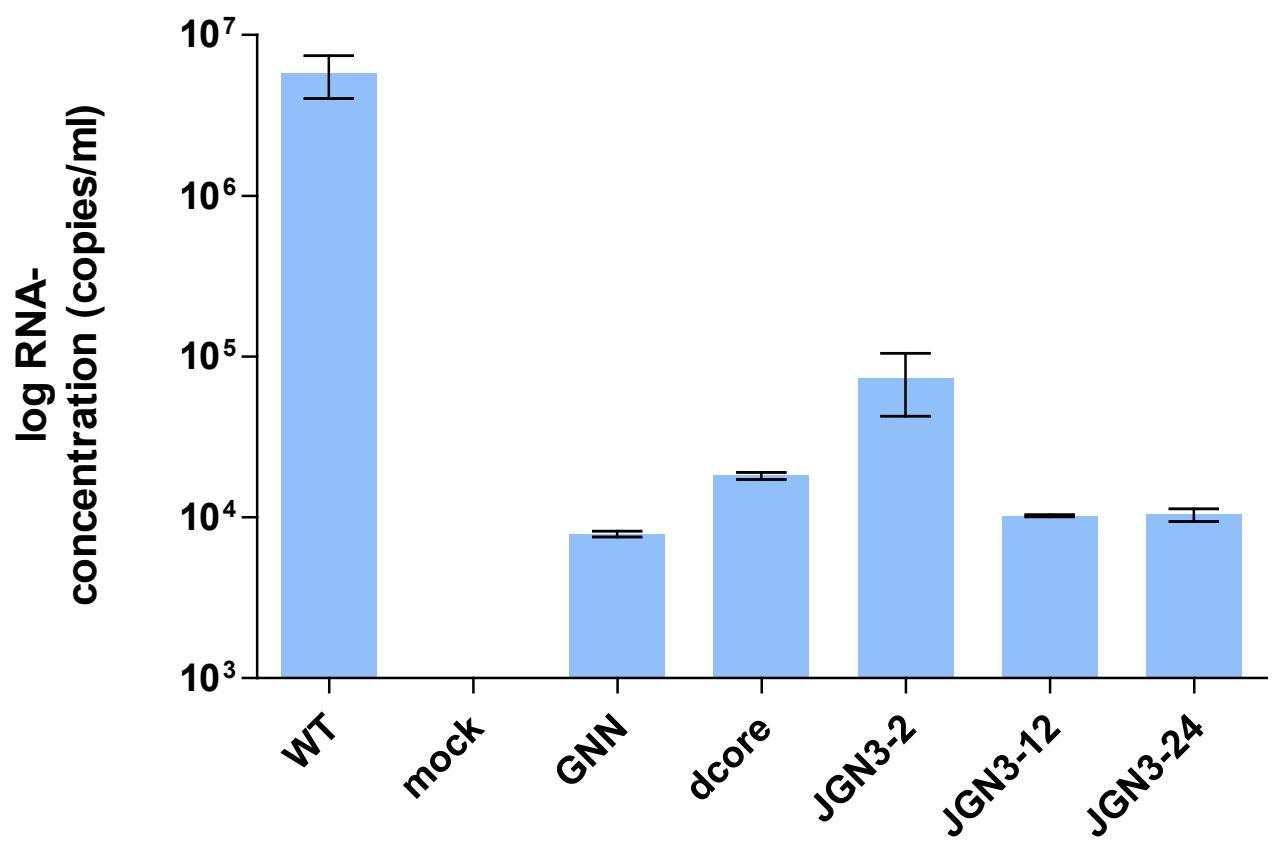

Figure 35 RNA release of infectivity-defective NS3hel mutants. The amount of RNA was measured by quantitative RT-PCR $6 \mathrm{~d}$ post-transfection. Results represent at least three individual rounds of experiments. Error bars show standard deviation of the mean.

Measurements showed that WT Jc1/GLuc2 efficiently released viral particles into the cell culture medium. An average amount of $5.7 \times 10^{6} \mathrm{copies} / \mathrm{ml}$ was release into the medium of cells transfected with WT RNA. Mock control did not release viral RNA into the cell culture medium. Replication defective control GNN released a mean of 7885 RNA copies/ml into the cell culture medium. An average of 18150 RNA copies/ml was measured in supernatant from cells transfected with $\Delta$ core which lacks the production of viral particles. Within medium of cells transfected with JGN3-2 RNA an average of $73700 \mathrm{HCV}$ RNA copies/ml could be detected. Within medium of cells transfected with RNA of JGN3-12 a mean value of 10223 
copies/ml was measured. An average of 10363 RNA copies $/ \mathrm{ml}$ could be meted in supernatant collected of cells transfected with JGN3-24 RNA. In comparison to WT all studied constructs revealed RNA levels 100 fold lower than WT levels.

\subsubsection{Intracellular infectious particles}

To determine whether the intracellular formation of viral particles of the mutants is intact, intracellular infectivity was compared to extracellular infectivity of the supernatants. HCV particles were shown to be assembled intracellular. Particles are thought to undergo maturation within the cell and are released afterwards as the buoyant density of intracellular particles differs from the one of mature extracellular virions (Gastaminza et al. 2006). To resolve if mutations within RNA of JGN3-2, JGN3-12 and JGN3-24 influence the intracellular formation of virus particle release cells were harvested $48 \mathrm{~h}$ post-transfection, freeze-thawed a couple of rounds and the cell lysate was used to infect naïve Huh 7.5 cells ('Material and Methods' p. 39).

Comparing intracellular and extracellular infectivity of the mutants, no significant difference between both could be shown regarding all studied constructs. WT Jc1/GLuc2 GLuc activity values were $3.1 \times 10^{7} \mathrm{RLU}$ when measured extracellular, intracellular values showed a mean of $1.8 \times 10^{6} \mathrm{RLU}$. Non infectious controls mock, GNN and $\Delta$ core showed average GLuc activities around $2 \times 10^{3} \mathrm{RLU}$ screening intracellular as well as extracellular infectivity.

Domain 3 mutant JGN3-2 did not show significant difference comparing intracellular and extracellular infectivity. Mean values were $4.7 \times 10^{4} \mathrm{RLU}$ when measuring intracellular infectivity and $1.3 \times 10^{4} \mathrm{RLU}$ measuring extracellular infectivity. Intracellular and extracellular infectivity of JGN3-12 did also not reveal significant differences. Mean intracellular GLuc activities were $9.1 \times 10^{3} \mathrm{RLU}$. Compared mean extracellular values were $1 \times 10^{4} \mathrm{RLU}$. Mutant JGN3-24 with changes within NS3hel domain 1 showed similar results. Intracellular infectivity levels were $4.1 \times 10^{3} \mathrm{RLU}$ and did not differ significantly from extracellular GLuc activity values of $5 \times 10^{3} \mathrm{RLU}$.

All scanned mutants could not infect naïve Huh7.5 cells neither when using extracellular cell culture medium nor using intracellular cell lysates. 


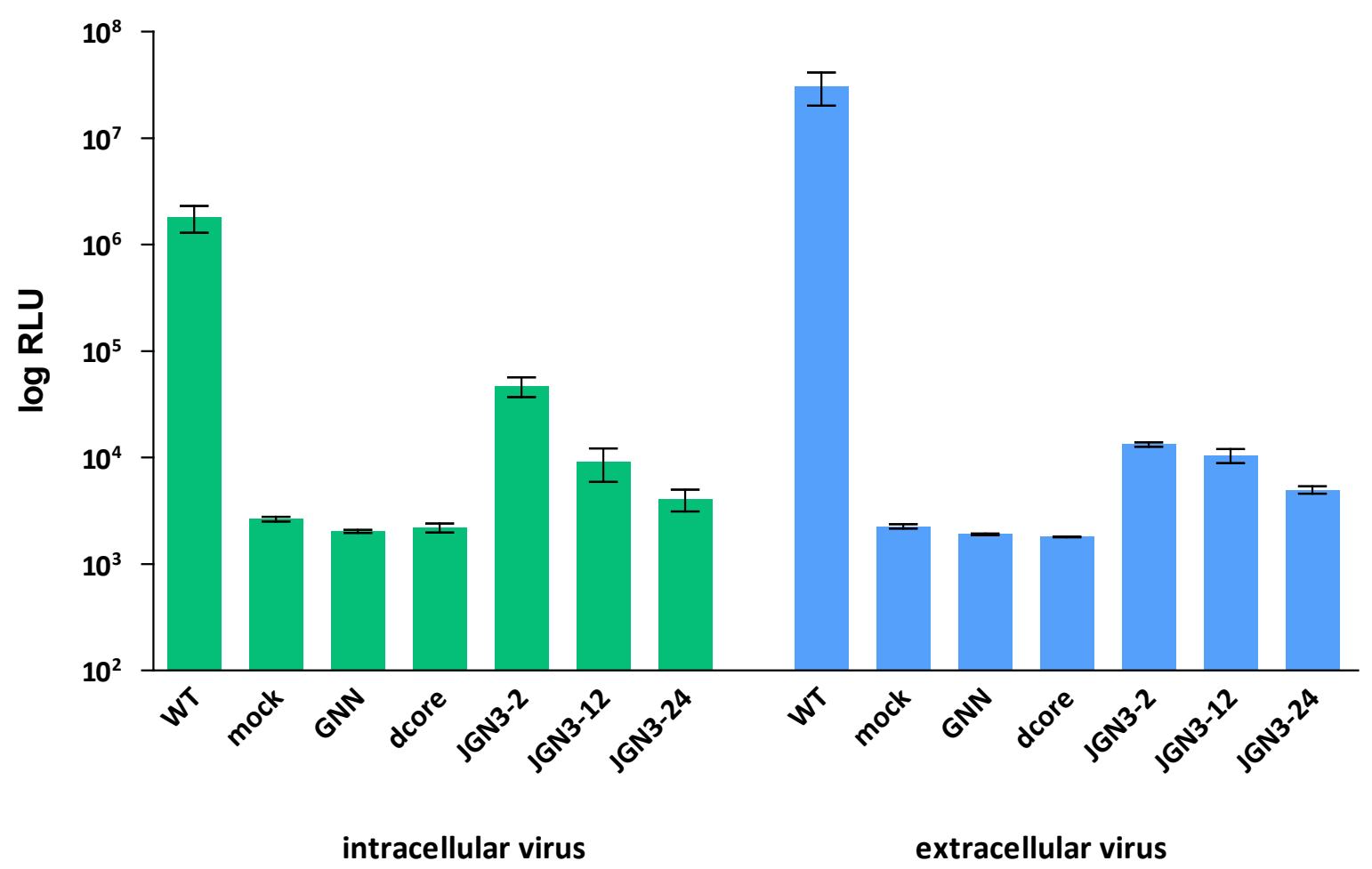

Figure 36 Intracellular infectivity vs. extracellular infectivity of NS3hel mutants. Cells were transfected with RNA of the mutants, 48h post-transfection cells were lysed and used to infect native Huh 7.5 cells. Results represent at least three individual rounds of experiments. Error bars represent standard deviation of the mean.

\subsubsection{Physical interactions of NS3hel with NS2}

The Lindenbach laboratory works on the identification of a complex which is essential for virus particle assembly and contains NS3, NS2 and the envelope glycoproteins. The involvement of NS2 in this complex as a coordinator between E1 and E2 and NS3 was published recently (Stapleford and Lindenbach 2011). As this complex is shown to be essential for HCV assembly, the involvement of NS3hel in this complex is interesting to determine and was studied in this thesis. Whether the changes T612A, H613A, T537A, H540A, H541A and A217R within constructs JGN3-2, JGN3-12 and JGN3-24 influence the formation and stability of the NS2-NS3 complex was studied.

To check interactions between NS2 and NS3hel pulldown experiments were performed. Constructs expressing the biotin acceptor peptide (BAP) at the amino-terminal end of NS2 were used. High-affinity interactions between biotin and streptavidin can be used to isolate proteins as described in the literature. The previously described mutations within JGN3-2, JGN3-12 and JGN3-24 were cloned into BAP-expressing full length HCV constructs named Jc1/bioNS2. Furthermore, Huh 7.5 cells expressing the E.coli biotin ligase BirA were transfected with RNA of the Jc1/bioNS2 mutants. WT Jc1/bioNS2 and mock, GNN and $\Delta$ core controls were used as well. Expression of the E.coli biotin ligase BirA results in the addition 
of a biotin molecule to the lysine residue of the BAP at the amino terminal end of NS2. This biotin molecule can interact with streptavidin to separate NS2 and proteins that are complexed with NS2. Stapleford and Lindenbach (2011) showed that the BAP-containing constructs do not influence viral replication or infectivity compared to untagged Jc1. 48 hours post-transfection cells were lysed. The lysate was mixed with streptavidin magnetic beads and separated on a magnetic rack to capture biotinylated proteins. NS2 and proteins that are bound to NS2 were purified from the remainder proteins of the cells. Proteins were separated by SDS-PAGE and transferred to PVDF membranes. Indicated proteins were immunoblotted. This system was used to determine whether assembly defective NS3hel mutants physically interact with NS2 or if the mutations inhibit these interactions.

All experiments were done with Huh 7.5(BirA) cell lysates harvested 48h post-transfection. Total cell lysate was blotted to show proteins present within the lysate prior to protein separation. This was compared to the cell lysate after protein separation with the previously described system.

Within total cell lysates both proteins NS2 and NS3 could be detected prior to protein separation within cells transfected with WT Jc1/BioNS2 as well as with mutants JGN32/BioNS2, JGN3-12/BioNS2 and JGN3-24/BioNS2. Proteins NS2 and NS3 migrated with molecular masses of $24 \mathrm{kDa}$ (NS2) and $70 \mathrm{kDa}$ (NS3). Results confirmed that these proteins were present as viral replication is intact.

WT Jc1 represents a control that did not include the BAP at the amino terminal end of NS2. Untagged WT Jc1 showed a distinct band at $23 \mathrm{kDa}$ (NS2) lacking the AP-sequence and at $70 \mathrm{kDa}$ (NS3). Mock control did not show any band as it did not include any HCV proteins.

All of the screened mutants and controls produced proteins NS2 and NS3. An equal amount of produced proteins compared to the WT Jc1/BioNS2 could be detected. The mutants efficiently replicated and produced viral proteins.

After protein separation of NS2 and NS2-bound proteins, WT Jc1/BioNS2 showed a distinct band at $24 \mathrm{kDa}$ (NS2). NS3 was also present in the lysate after separation. A distinct band was detected at $70 \mathrm{kDa}$. Both, NS2 and NS3 could be captured and detected with the described system. As just NS2 including the BAP can be bound to the streptavidin beads NS3 had to be bound to NS2 to show up after separation.

Furthermore, constructs JGN3-2/BioNS2, JGN3-12/BioNS2, JGN3-24/BioNS2 included the tagged NS2 and could be successfully biotinylated and separated with the streptavidin system.

JGN3-2/BioNS2 with mutations T612A and H613A within NS3hel domain 3 also showed a distinct band at $24 \mathrm{kDa}$ detecting NS2. Furthermore, NS3 could be detected migrated in the 
gel with a molecular mass of $70 \mathrm{kDa}$. The NS3 band of bound JGN3-2/BioNS2 is comparable to WT Jc1/BioNS2.

Bound JGN3-12/BioNS2 including mutations T537A, T540A and H541A showed a distinct band at $24 \mathrm{kDa}$ (NS2). Detected NS3 band at $70 \mathrm{kDa}$ showed a significant difference to WT Jc1/BioNS2. A weak band could be detected but it appears thinner compared to bound WT Jc1/BioNS2.

JGN3-24/BioNS2 with mutation A217R within NS3hel domain 1 also showed a strong band at $24 \mathrm{kDa}$ detecting NS2. However, NS3 detecting band at $70 \mathrm{kDa}$ is also significantly slighter than the bound WT band.

Results were controlled by WT Jc1 and mock. Untagged NS2 within the Jc1 construct was not captured after separation as it cannot be biotinylated. Mock controls remained negative in both total cell lysate and lysate after separation as cells were electroporated without RNA.

Mutations within the mutants JGN3-12 and JGN3-24 did influence NS2-NS3 interactions but did not completely disrupt these. However, mutant JGN3-2 seems to not influence proteinprotein interactions between NS2 and NS3.

Total
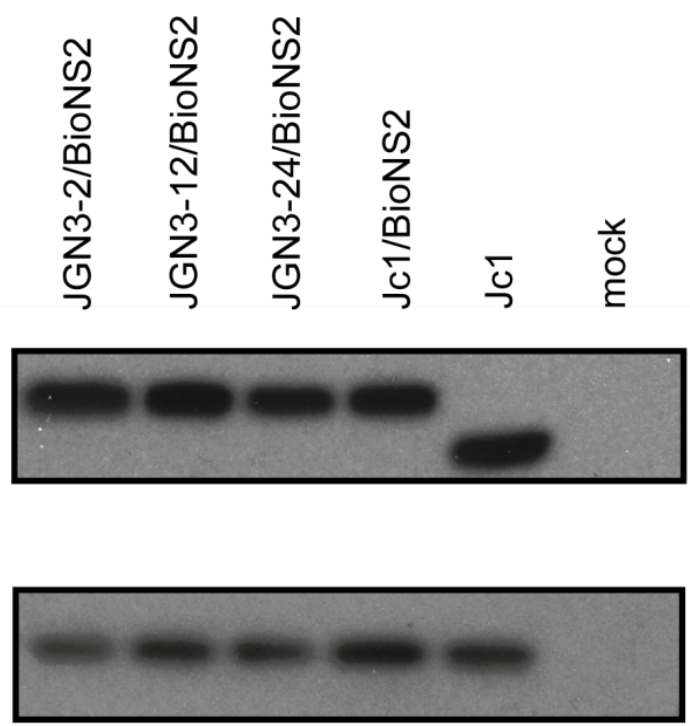

Bound

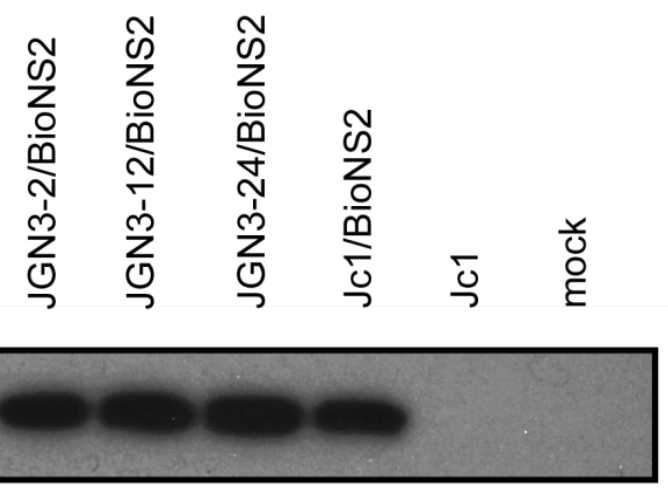

NS2

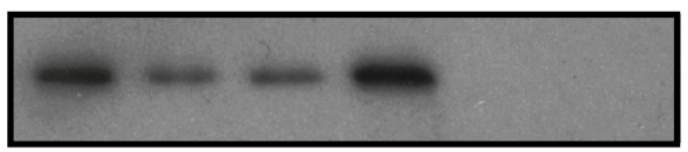

Figure 37 Pulldown of NS2(AP)-containing complexes. Cells were transfected with Jc1/BioNS2 complexes and Jc1. Biotinylated proteins were separated with streptavidin beads, eluted in SDS-PAGE sample buffer, separated by SDS-PAGE and immunoblotted for NS2 and NS3, as described in 'Material and Methods' ( $p$. 43ff). Total shows proteins present in the cell lysate before separation. Bound shows proteins separated by streptavidin beads. Results were confirmed by three individual rounds of the experiment. One of these rounds representative for all is shown here. 


\subsection{Long term infectivity - passaging experiments over 12d}

To determine the characteristics of HCV constructs JGN3-2, JGN3-12 and JGN3-24 mutants were passaged over a period of more than 96h. Supernatants of transfected Huh 7.5 cells were collected at $24 \mathrm{~h}, 48 \mathrm{~h}, 72 \mathrm{~h}, 96 \mathrm{~h}, 6 \mathrm{~d}, 9 \mathrm{~d}$ and $12 \mathrm{~d}$ to infect native Huh 7.5 cells. Phan et al. (2009) described that the Jc1/GLuc construct is stable for up to 4 weeks so the method of measuring GLuc activity can be further used as it is still reliable.

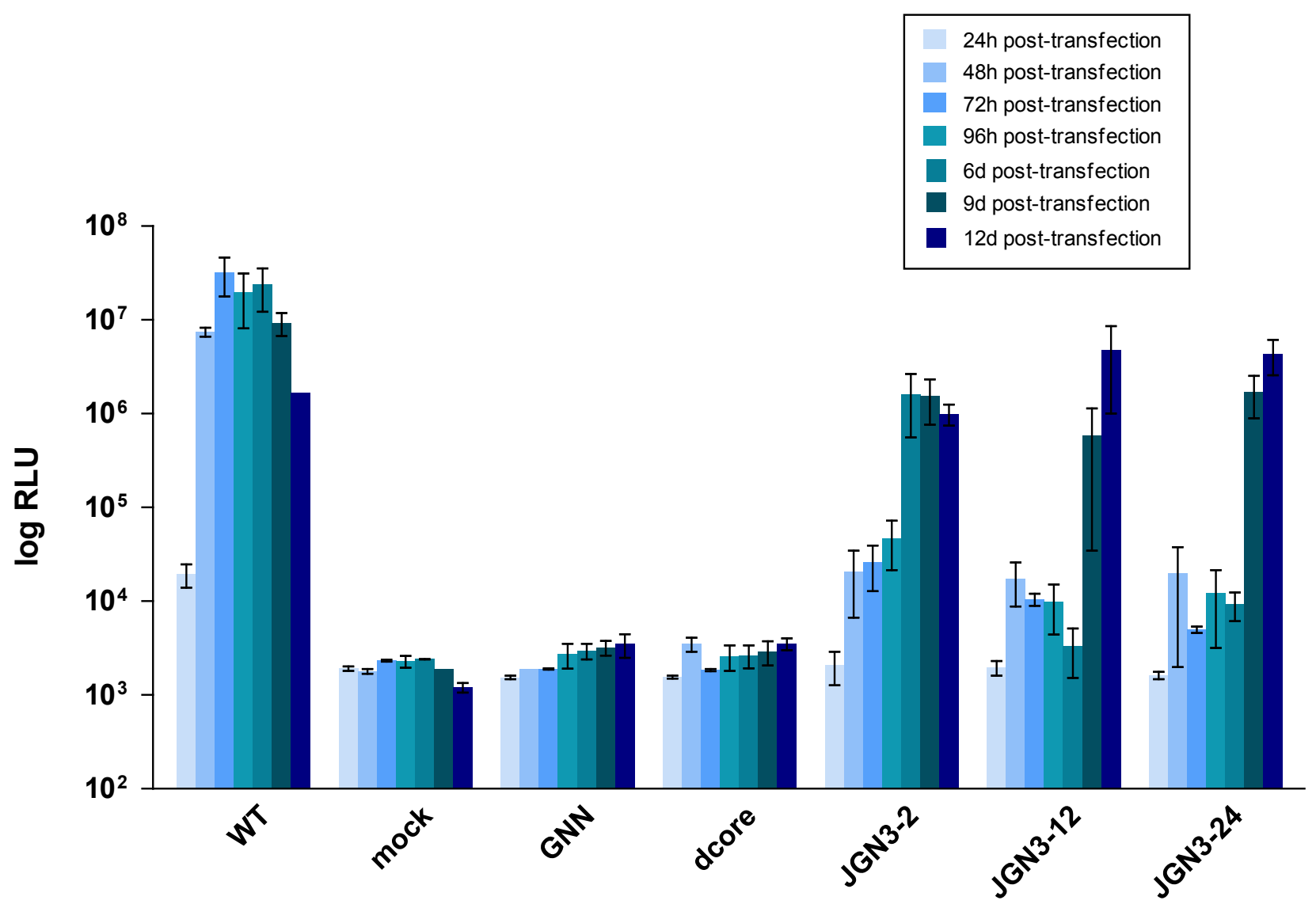

Figure 38 Infectivity of JGN3-2, JGN3-12 and JGN3-24 over time. Results represent at least three individual rounds of experiments. Error bars represent standard deviation of the mean.

Supernatant of JGN3-2 including mutations T612A and H613A infected native Huh 7.5 cells $6 \mathrm{~d}$ post-transfection. GLuc values ranged between $1.6 \times 10^{6}$ and $1 \times 10^{6} \mathrm{RLU}$ at time points 6 to 12 days post-transfection.

Mutants JGN3-12 and JGN3-24 regained infectivity after an average of 9d. GLuc levels of JGN3-12 were $5.8 \times 10^{5}$ and $4.8 \times 10^{6}$ RLU 9 and 12 days post-transfection. Levels of JGN3-24 were $1.7 \times 10^{6}$ and $4.3 \times 10^{6} \mathrm{RLU} 9$ and 12 days post-transfection. Results were confirmed by anti-NS5a staining (data not shown). 


\subsection{Summary of results}

$25 \mathrm{HCV}$ constructs with changes in NS3 helicase domains were studied in replication and infectivity. 16 mutants showed severe or moderate defects in RNA replication. 6 mutants were able to efficiently replicate and produce infectious virus similar to WT values. Three mutants were able to replicate but had severe defects in assembly.

Several residues could be mapped to be essential for viral genome replication. All changes made within NS3 domain 2 lead to severe defects in viral replication. Residues E346, P348, F349, Y350 and P355 within domain 2 were mapped to be essential for replication. Within domain 3 residues E530, E533, D543 and H545 were shown to be involved in replication as well as Y618. A couple of other residues in domain 3 seem to influence replication as constructs showed reduced replication. These residues within domain 3 included L588, K589, P590, T591 and Y618. Within NS3 domain 1 several changes also influenced HCV RNA replication. Residues Q221, G222, Y223, D308, Q309, E311 and T312 could be mapped playing a role in viral replication.

NS3 domain 1 and 3 were shown to be involved in HCV virus particle assembly. Residues T537, T540, H541, T612 and H613 within domain 3 and A217R in domain 1 were identified influencing virion assembly. T612 and H613 influenced reaction kinetics. Changes of T537, T540, H541 and A217 induced a severe defect in particle assembly.

Within this thesis NS3 domain 1 and 3 could be shown to be essential for virus particle assembly at an early step of the assembly process prior to release and formation of intracellular infections particles.

NS3 physically interacts with NS2. Changes within domain 1 and 2 could be shown to influence interactions but not to inhibit them completely.

Furthermore, the effect of the lack of HCV in RNA proofreading and its high mutation rate could be demonstrated. The virus is able to regain infectivity in cell culture after long term incubation. 


\section{Discussion}

Hepatitis C virus non structural protein 3 is a multifunctional protein with important roles within the viral life cycle. It is essential for viral replication as a part of the membraneassociated replicase complex. NS3 contains protease, NTPase and helicase activities necessary for viral replication. Next to its role in replication it is also suggested that NS3 helicase domain is involved in virus particle assembly.

The dual role of the NS3 helicase domain in the viral life cycle is demonstrated by the data presented here.

\subsection{Role of NS3hel in viral replication}

Helicases are involved in replication by binding and unwinding double-stranded DNA or RNA making the templates accessible for replication. Single-stranded Hepatitis $\mathrm{C}$ viruses also require helicase activity for RNA replication. Even though the precise role of Hepatitis C NS3 helicase is still unclear, it was previously shown to be essential for viral RNA replication (Kolykhalov et al. 2000; Lam and Frick 2006). It is hypothesized that single-stranded RNA viruses require helicase activity to separate partially double-stranded replication intermediates or pairs between templates and their nascent complementary strands (Kadaré and Haenni 1997). Within this study several residues within all three major NS3 helicase domains could be identified playing a role in viral replication. Mutational changes of these residues in the context of the genotype $2 a$ full length replicon system lead to defects in viral replication.

As helicase activity is essential for replication, defects must somehow influence enzymatic activities of NS3hel. Induced changes of surface residues tried to avoid previously described sites that are essential for helicase activity like the conserved helicase motifs and NTP and nucleic acid binding sites. Even though these sites were avoided mutations could have lead to changes in protein conformation or intra-molecular interactions. Another explanation for the observed replication defects could be extra-molecular interactions. Earlier studies revealed interactions between NS3 and the RdRp NS5B, NS5A and NS4B. They are supposed to form a membrane-associated replication complex which is a hallmark of positive-strand RNA viruses. In addition, it is described that NS3-4A protease enhances NS3 helicase activity suggesting its involvement in the replication complex (Zhang et al. 2005; Gu, B et al. 2005). Gosert et al (2003) described that NS4B is capable of forming a complex with NS3 suggesting it might play a role in recruiting NS3 to the site of RNA replication, the socalled membranous web. Moradpour et al. (2004) showed that NS3, NS4B and NS5B co- 
localize within the cell. Zhang et al (2005) described that NS3-4A protease might mediate NS3hel-NS5B-interactions during replication. Mutated NS3hel surface residues as studied here might influence protein-protein interactions resulting in replication defects.

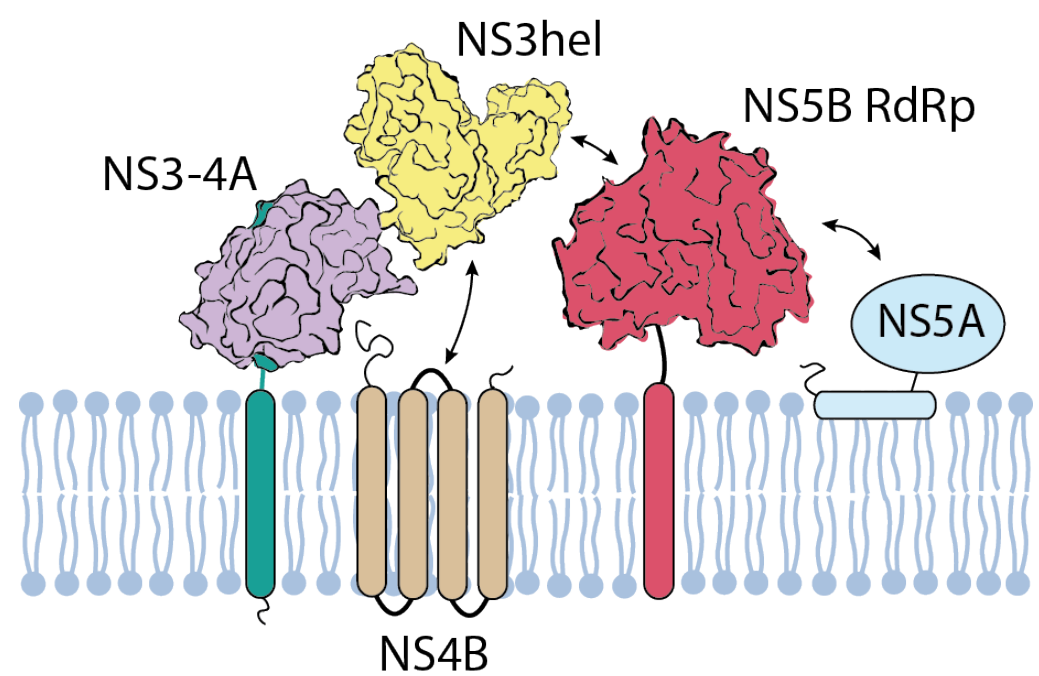

Figure 39 Replication complex. Suggested protein-protein interactions are indicated with arrows.

Within NS3hel domain 3 data presented here revealed that $\alpha$-helices 15, 16, 20 and 21 play a role in HCV replication. Residues Y618, L588, K589, E530, E533, D543, H545, D543 and H545 were identified being involved in replication.

Only a couple of residues within domain 3 essential for HCV replication were described in the literature so far. Previous studies of Lin and Kim, JL (1999) reported domain 3 residue W501 within $\alpha$-helix 13 to be important in the replication process by anchoring the nucleic acid to the active centre of the enzyme. Lam and Frick (2006) performed further studies with several NS3hel residues including W501. They described that the aromatic side chain of W501 is essential for unwinding RNA supporting replication. Another residue within domain 3 described by Lam and Frick is E493 which is located in the same a-helix as W501 and also contacts the nucleic acid. Furthermore, domain 3 residues H528, F531, W532 and F536 within a-helix 15 were described to be important for proper NS3 protein folding (Lam et al. 2003).

Within experiments of this thesis two residues important for HCV replication could be identified within alpha-helix 15. E530 and E533 were located in this area. These findings underline that $\alpha$-helix 15 is important for the active conformation of NS3hel. Furthermore, $\alpha-$ helix 15 is located right beside $\alpha$-helix 13 with residues W501 and E493 that were shown to interact directly with the nucleic acid. Though the role of $\alpha$-helix 15 might be to stabilize the conformation of NS3hel, especially stabilizing $\alpha$-helix 13 and the nucleic acid binding groove. 
Moreover, residues D543 and H545 within a-helix 16 influence viral replication. These residues might also stabilize the conformation of $\alpha$-helix 13 and the nucleic acid binding groove as $\alpha$-helix 16 is also lining $\alpha$-helix 13 maybe stabilizing both $\alpha$-helix 15 and 13 . But due to its location $\alpha$-helix 16 might also interact directly with the nucleic acid. To determine whether or not these residues interact with RNA or DNA further studies would be necessary.
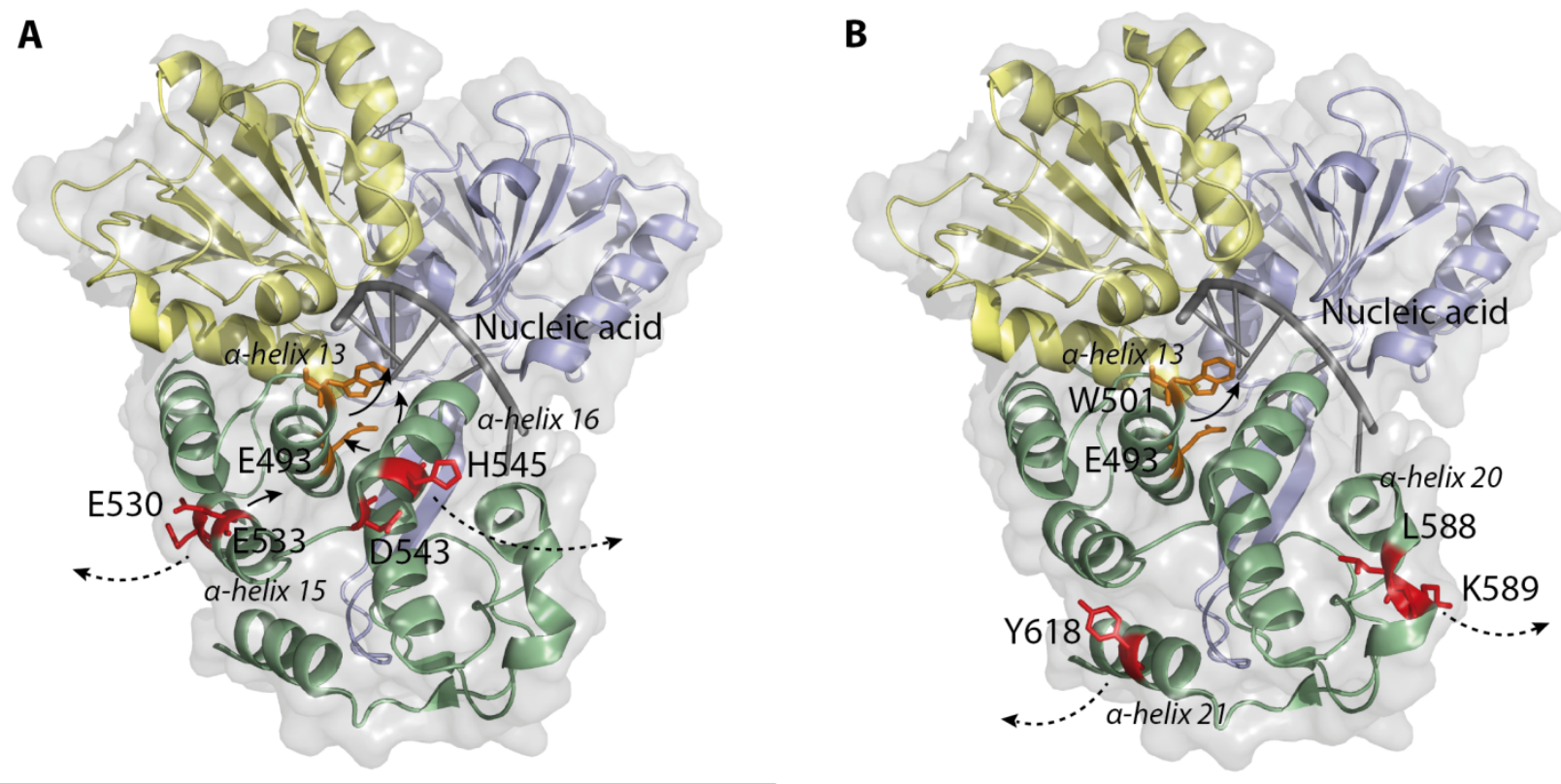

Figure 40 Involvement of studied domain 3 residues in replication. NS3hel domains 1, 2 and 3 are marked yellow, blue and green. Residues studied here are marked red and residues implicated in replication elsewhere are highlighted orange. Arrows indicate hypothesized interactions with the nucleic acid, with the NTP or intramolecular interactions. Dashed arrows indicate extramolecular protein-protein interactions. Models were rendered with PyMOL, Protein Data Bank code 3KQL. (A) Residues within alpha helix 15 and 16 implicated in replication. A-helices 15 and 16 might stabilize active conformation of the nucleic acid binding cleft or interact with other NS proteins. (B) Residues within alpha helix 20 and 21 implicated in replication. A-helices 20 and 21 are likely important for protein-protein interactions during replication.

However, studied surface residues Y618 within a-helix 21 and L588 and K589 within a-helix 20 are located more turned away from $\alpha$-helix 13. It is a possibility that they also stabilize $\alpha$ helix 13 , but they could more likely be considered to be essential to form interactions between NS3 and other NS proteins. Co-localization of NS3 and NS5B as well as with NS4B is described in the literature but no residues involved in these interactions were described so far (Ishido et al. 1998; Piccininni et al. 2002).

Interestingly, within this thesis, changes P590A and T591A in a-helix 20 that did not influence replication, restored defects of L588A and K589A when all four residues were combined in one mutant (JGN3-5). They seem to compensate conformational changes or charges fixing the replication defect.

As shown in this thesis NS3hel domains 1 and 2 were especially sensitive for changes. These findings underline their superior role in HCV replication. 
Conserved helicase motifs I, la, Y, Ib, Ic, II, and IIII are located within domain 1. Motifs V, IVa, $\mathrm{V}$ and $\mathrm{VI}$ are located within domain 2. Both domains are important for NTPase activity and nucleic acid binding. Earlier studies were able to reveal residues important for NS3 helicase activities located in domains 1 and 2. Those studies focused on residues at RNA binding and NTPase activity sites. Examined residues influence helicase activity by decreasing nucleic acid binding or NTPase activity. Lin et al. (1999) revealed several residues that appear to stabilize interactions between NS3hel and the nucleic acid. $\mathrm{Y}-\mathrm{OH}$ groups of T269 of domain 1 and T411 of domain 2 could be identified to be essential for nucleic acid binding. Unwinding activity and NTPase stimulation was abolished by changing these residues (Lin and Kim, JL 1999). W-501 of domain 3 (as described above) and V432 of domain 2 were further studied by different groups (Preugschat et al. 2000; Tai et al. 2001). These residues are implicated of being essential for unwinding activity. Considering the inchworm model these residues are believed to act as an anchor for the nucleic acid during conformational changes of the enzyme. Furthermore Tai et al. (2001) identified other residues essential for helicase activity. Residues within conserved Walker $A$ and Walker $B$ motifs as well as residues not located in these motifs were shown to be crucial for NS3hel ATPase activity. Domain 1 residues K210, D290, E291, C292 and H293 within the conserved motifs revealed a complete loss of ATPase activity when mutated. Outside these motifs residues T322, T324 within domain 1 and Q460 and R461 within domain 2 could be identified to be important for NTP hydrolysis. Furthermore, additional conserved motifs essential for DNA/RNA binding and strand separation were described by Lam et al. (2003). Therefore an Arg-clamp and a Phe-loop structure were described within domain 2. Residue R393 was shown to clamp RNA into the implicated binding cleft in presence and absence of ATP. Furthermore, interactions between F438 and F444 with domain 3 residues H528, F528, W532 and F536 were described to be important for proper protein folding (Lam et al. 2003).

Studying NS3hel domain 1 and 2 here affirmed the critical role of these domains in helicase activity. Within domain 2 residues E346, P348, F349, Y350 and P355 within beta sheet E1 as well as adjacent residues S342, S343 and T344 could be identified being essential for replication. Beta sheet E1 is located relatively close to T411 and R393 that were identified to interact with nucleic acids. Residues within $\beta E 1$ might influence conformation of the nucleic acid binding region. Next to being involved in replication by stabilizing active protein conformation studied domain 2 residues could also be involved in protein-protein interactions with other NS proteins.

Within domain 1 residues D308, Q309, E311 and T312 located within a-helix 8 on the surface of the back of domain 1 as well as residues Q221, G222 and Y223 were shown to be essential for viral replication. Residues are outlying of previously described sites identified being involved in NTPase activity or nucleic acid binding. Residues might be important for 
proper protein folding. But it is also likely that they interact with other NS proteins. To determine between these possibilities further studies have to be done but were not subject of this thesis. Interestingly, mutation Q221L was previously described as a cell culture adaptive mutation. It was found to enhance virus production in cell culture. Furthermore it was shown to suppress assembly defects in NS2 (Ma et al. 2008; Phan et al. 2009). This site seems to influence functional RNA binding of NS3-4A. Phan et al. (2009) hypothesized that Q221 might influence RNA-stimulated interactions of the ATP-binding pocket.
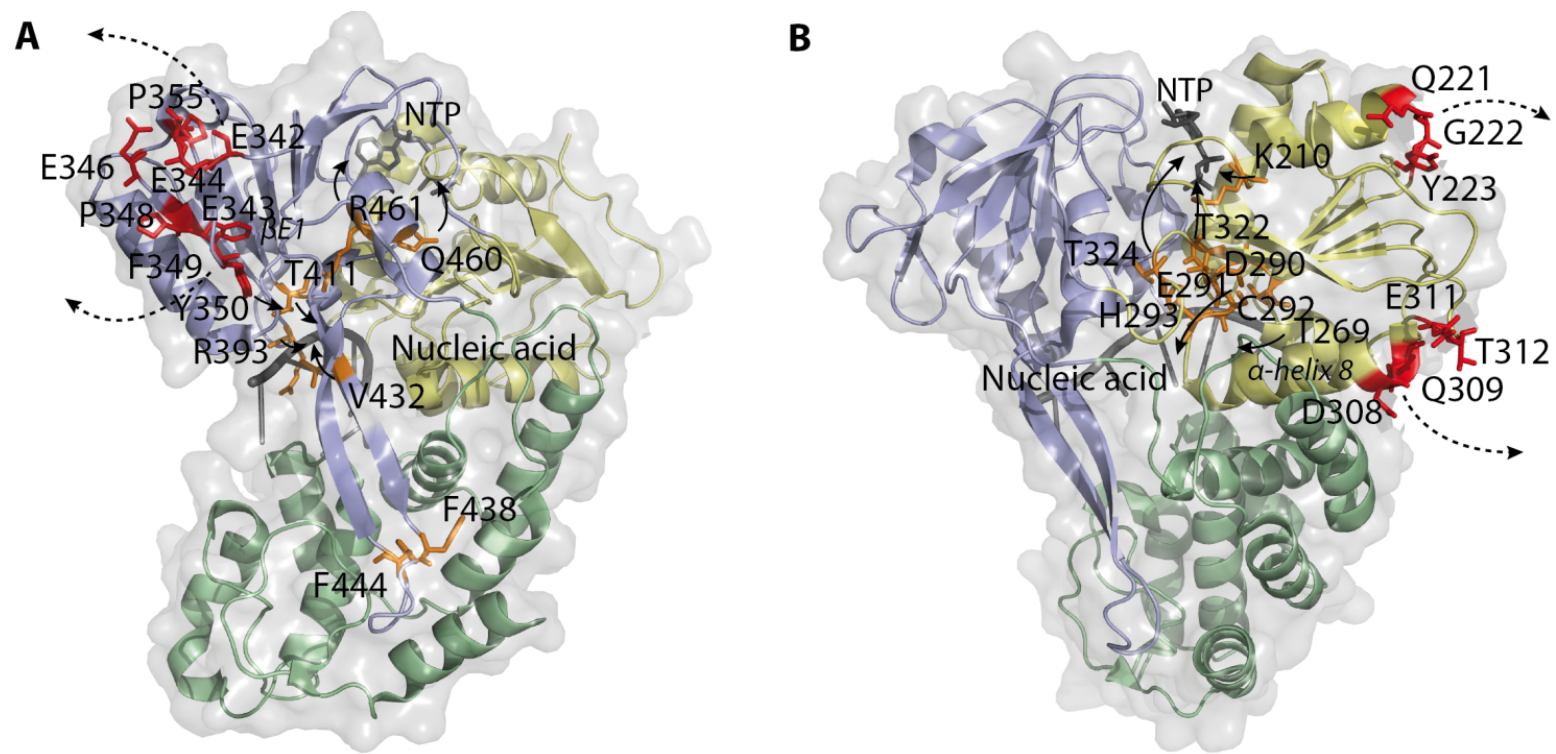

Figure 41 Involvement of studied domain 1 and 2 residues in replication. NS3hel domains 1,2 and 3 are marked yellow, blue and green. Residues studied here are marked red and residues implicated in replication elsewhere are highlighted orange. Arrows indicate hypothesized interactions with the nucleic acid, with the NTP or intramolecular interactions. Dashed arrows indicate extramolecular protein-protein interactions. Models were rendered with PyMOL, Protein Data Bank code 3KQL. (A) Domain 2 residues implicated in replication. While residues S342, S343, T344, E346, P348, F349 and P355 are likely playing a role interacting with other NS proteins during replication, Y350 might also play a role in stabilizing active conformation of the nucleic acid binding cleft. (B) Domain 1 residues implicated in replication. Residues Q221, G222, Y223, D308, Q309, E311 and T312 seem to be involved in protein-protein interactions.

\subsection{Infectivity}

Infectivity screenings confirmed that without efficient replication infectious particles cannot be produced as no viral RNA can be packaged. However, several residues that did not or just minimally influence replication and infectivity, could be identified to be silent mutations. Sites are not involved in both functions. Residues D503, E508, R512, S548, Q552, P614 and K617 do not influence infectivity capability. Interestingly, mutant JGN3-5 that included mutations of residues L588, K589, P590 and T591 was replication competent and infectious comparable to WT. But mutant JGN3-6 that included only L588 combined with K589 was not able to replicate or to be infectious. JGN3-7 including P590 combined with T591 was able to 
replicate and to be infectious but activity of both functions was decreased. The combination of all four mutations restores defects of L588 and K589 alone.

The most striking findings within the infectivity experiments were represented by JGN3-2, JGN3-12 and JGN3-24. These constructs, including changes of residues A217, T537, T540, H541, T612 and H614, were able to replicate but lacked the ability to infect naïve Huh7.5 cells. As the replication machinery could be shown to be intact, these constructs could be perfectly used as models to study HCV particle assembly. Changes have to interfere the production of infectious viral particles at a post-replication step within the virion production pathway. Therefore, the problem has to be either in the formation of viral particles or the release of infectious virions.

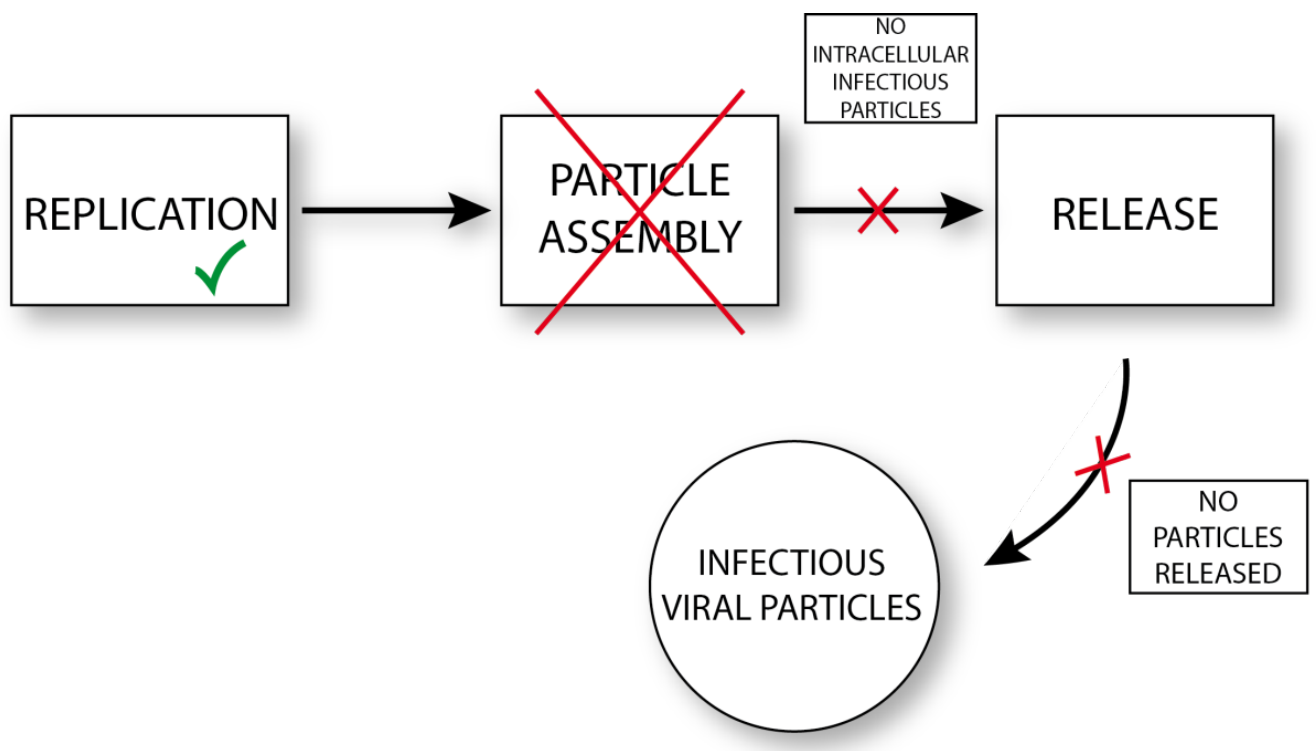

Figure 42 NS3hel is essential for an early step of particle assembly prior to the formation of intracellular viral particles. The figure summarizes steps essential for the formation of infectious viral particles and defects caused by studied mutations. As replication is intact, a post-replication step has to be blocked within JGN3-2, JGN3-12 and JGN3-24.

\subsection{JGN3-2, JGN3-12 and JGN3-24 - constructs that were able to replicate but not to be infectious}

To determine whether the lack of infectivity of JGN3-2, JGN3-12 and JGN3-24 is attributable to defects in the release of infectious viral particles or in the process of particle assembly further experiments were performed.

By using qRT-PCR studies the amount of viral RNA released into the cell culture medium was measured assuming that a lack of viral RNA within the medium points out a defect in assembly or release. If higher RNA levels can be found one can assume that viral particles are released but virions are not infectious which might point out a problem in the entry process within the viral life cycle. Results revealed that WT was able to replicate RNA and assembled and released infectious virions. The mock control did not release viral RNA into 
the cell culture medium as cells were transfected with no viral RNA. Replication defective control GNN and assembly defective control $\Delta$ core are not able to release viral particles. The low amount of viral RNA measured within the cell culture medium is caused by residual leftover from the transfection. Another possible cause was described by Lindenbach et al. (2005) and other groups. HCV replicons which are able to replicate, release small amounts of viral RNA into the cell culture (Wakita et al. 2005). This might explain, why the level of RNA within the medium of replication competent $\Delta$ core was slightly higher compared to replication defective GNN. JGN3-2, JGN3-12 and JGN3-24 show an as low amount of viral RNA comparable to the negative controls. These mutants with mutations T612A, H613A, T537A, T540A and H541A and A217R did not release HCV RNA into the cell culture medium. JGN-2 showed a slightly higher amount of particles released six days posttransfection. Defects might have been already restored by other mutations in some of the experimental rounds carried out. Alternatively, a higher amount of RNA could have been secreted into the media.

Altogether, one can say that none of the three constructs was able to release viral particles showing that the defect can be found within the process of particle assembly or the release process itself. To determine between these possibilities further experiments were performed. To find out whether intracellular infectious HCV particles are produced within the cells transfected with the replication competent constructs, intracellular infectivity experiments were carried out. Results revealed that none of the examined mutants was able to infect Huh7.5 cells showing that no infectious intracellular particles are produced. These results implicate that NS3hel is essential for an early step of virus assembly prior to the formation of intracellular infectious particles.

\subsubsection{NS3hel and virus particle assembly}

The process of HCV particle assembly could not be unraveled to date. Also the complete role of the NS3 helicase is not known. Recent studies revealed a novel role for the NS3 helicase domain in virus particle assembly. Single mutation Q221L within NS3hel domain 1 was shown to compensate NS2 defects in virion production (Ma et al. 2008; Phan et al. 2011). Furthermore, Ma et al. described mutation I286V within NS3hel domain 1 to restore changes within domain 2 (I399V). These studies revealed evidence for the involvement of the two major subdomains 1 and 2 of NS3hel in the process of HCV particle assembly.

In related helicases of flaviviruses and pestiviruses, like Kunjin virus (KUN), yellow fever virus (YFV) and bovine viral diarrhea virus (BVDV), the involvement of NS3 in assembly of infectious particles could be described (Agapov et al. 2004; Kümmerer and Rice 2002; Liu et al. 2002). 
Due to these findings alanine-scanning mutagenesis of residues located within all major NS3hel subdomains was performed within this thesis. Findings, based on experiments using the full-length Jc1 replicon-based cell culture system, report the essential role of NS3hel in virus particle assembly indentifying residues within domain 1 and 3 that are involved in this process.

Results presented here demonstrating that NS3 helicase domains 1 and 3 are involved in HCV particle assembly agree with previous finding of Ma et al. No novel residues within NS3 domain 2 involved in assembly could be revealed. However, the involvement of NS3hel domain 3 could be presented here for the first time.

Furthermore, the affinity purification system developed by the Lindenbach laboratory was used to study protein-protein interactions between NS2 and NS3. The efficiency of this system was described recently (Stapleford and Lindenbach 2011) NS2 and associated proteins could be captured in the context of genotype 2a virus producing Huh 7.5 cells.

Data presented here confirm Stapleford and Lindenbach (2011). NS2-NS3 interactions could be demonstrated and residues T537, T540 and H541 within domain 3 and A217 within domain 1 could be mapped influencing these interactions.

\subsubsection{How does NS3hel contribute to virus particle assembly?}

NS3hel mutations affecting virus assembly could be mapped on surface residues within domain 1 and 3 . It was also demonstrated that enzyme activities, NTPase and helicase activity, were not influenced by these mutations as replication was intact. Thus, these findings point to the assumption that NS3hel interacts with other HCV proteins or so far unidentified host cell proteins within the process of virus particle assembly.

Stapleford and Lindenbach (2011) revealed that NS2 physically interacts with E1, E2 and NS3. It is also thought that p7 and NS4A are a part of an NS2 complex. NS2 is described to coordinate interactions between E1-E2 with NS3-4A. It is assumed that these interactions within this complex play a central role in virus particle assembly.

On the basis of these results the role of NS3 helicase in virion assembly and especially in this complex was determined here. Assuming that assembly is based on this complex, disruption of the complex has to lead to a defect in the assembly of viral components leading to a lack of infectivity.

Further analysis of NS3 helicase residues which are implicated in viral assembly by mutational experiments in this study reveal whether the lack of infectivity is caused by influencing protein-protein interactions of the described assembly complex. With the newly described protein-complex purification system, interactions between NS2 and NS3 could be confirmed. Physical interactions between NS2 and wild-type NS3 were demonstrated. 
Furthermore, mutations T537A, T540A and H541A within domain 3 and mutation A217R within domain 1 revealed significant decreases in NS2-NS3 interaction levels. These residues that are proven to be essential for virus particle production affect protein-protein interactions. Affinity purification experiments revealed decreased levels of NS2-NS3 interactions, but a certain fraction of NS2-NS3 complexes were proven.

NS2 is thought to be stabilized by NS3 forming a dimeric proteolytically active NS2-3 protease conformation (Schregel et al. 2009). Interactions between NS2 and NS3 are further supposed to be involved in the formation of the replicase complex (Kiiver et al. 2006). These NS2-3 interactions cannot be excluded getting captured within the pulldown experiments which would explain these findings.

Whereas, another possible explanation might be that interactions between NS2 and NS3 are unstable but not all complexes are disrupted resulting in a significantly decreased amount of intact complexes leading to a lack of infectivity.

The involvement of NS3hel domain 1 and domain 3 in virus assembly could be determined in an early step of the assembly process prior to the formation of intracellular infectious virions assuming that the described complex is involved in assembly during this early step.

Previous studies suggested that cytoplasmic lipid droplets (LD) play a key role in the formation of viral particles. Core and LDs were described by Boulant et al. (2007) and Jones, DM and McLauchlan (2010) to be involved in initiation of viral assembly. Core was suggested to coat lipid droplets and recruit NS proteins and replication complexes to LD and associated membranes (Miyanari et al. 2007). Disrupted interactions between core and LD were shown to lack down particle assembly (Boulant et al. 2007). It has also been described that core affects the localization of LD within the infected cell (Lyn et al. 2010). Furthermore, NS5A could be localized on lipid droplets as well. Interactions between NS5A and LDs are further described being essential for particle assembly (Tellinghuisen et al. 2008). Miyanari et al. (2007) demonstrated that core is directly localized on the surface of lipid droplets while NS5A was mainly found within membranous structures surrounding lipid droplets that are coreassociated.

The membranous structures are assumed to be ER derivates as they were found near the ER around the nuclear membrane. These membrane alterations are described for HCV replication and assumed as a site for assembly (Bartenschlager et al. 2004). The corecoated lipid droplets associate with these ER membranes that include HCV proteins. This association is thought to be mediated by NS5A.

As the first step of assembly, core-coated LD and NS5A might build an initiation complex arranging assembly-essential components. The NS2-NS3 protein complex might arrange in close proximity of the core and NS5A associated LD. It might be important in another early step for initiation of viral assembly together with core, NS5A and lipid droplets (LDs). 
Proposed interactions might establish physical closeness between components that build the infectious particle, bringing together core and the ER membrane and a complex of E1-E2, while NS2 is important for coordination and NS3 handles the viral genome.

Next to their role of catalyzing the disruption of hydrogen bonds for unwinding duplex DNA or RNA, helicases are also described to move along nucleic acid strands in a polar way. King et al. (2001) proposed that DNA helicase complexes of adeno-associated virus type 2 can act as molecular motors that insert ssDNA into preformed capsids. Based on these findings one can assume that helicases are able to target single-stranded genomes to the sites of viral packaging, inducing integration of the genome into capsids or forming capsids around it. NS3 in a complex with NS2 and E1-E2 could function as a RNA binding protein that unwinds double stranded RNA and moves ssRNA to the site of endocapsid forming and induces the packaging of the viral genome. The protein complex could obtain the role of stabilizing interactions and building a physical closeness between components involved in particle assembly. Lorenz (2010) described that NS2 forms a dimer for catalytic active conformation. It is likely that NS2 is involved in viral assembly in its dimeric form coordinating E1-E2/NS3 interactions. Due to its relatively small size of $21 \mathrm{kDa}$ dimeric conformation might physically simplify maintaining the function as a linker between E1-E2 and NS3 but the actual conformation has to be determined in future studies. In flavivirus assembly NS2A was shown to induce membrane alterations and play a role in rearranging envelope proteins (Leung et al. 2008). HCV NS2 could play a similar role.

A possible model of the function of the NS2-NS3 complex is proposed here in which NS2 coordinates the interaction of NS3 with E1-E2 which induces the formation of core as a nucleocapsid around the viral genome. Figure 43 shows a model of HCV assembly including proposed protein-protein interactions. 
A

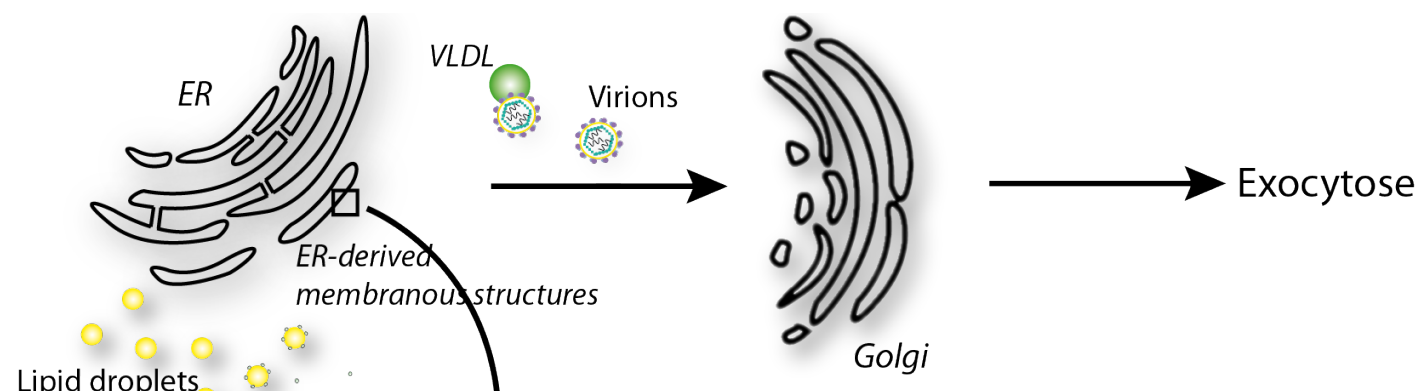

B

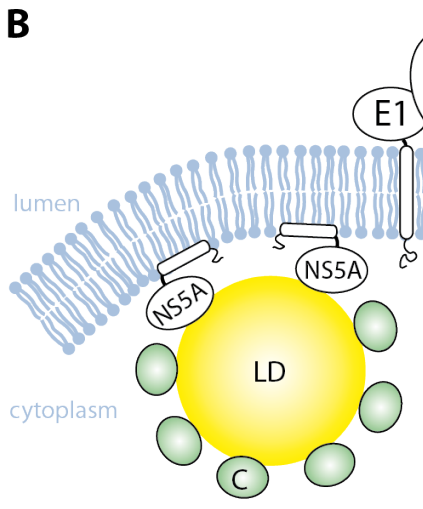

D
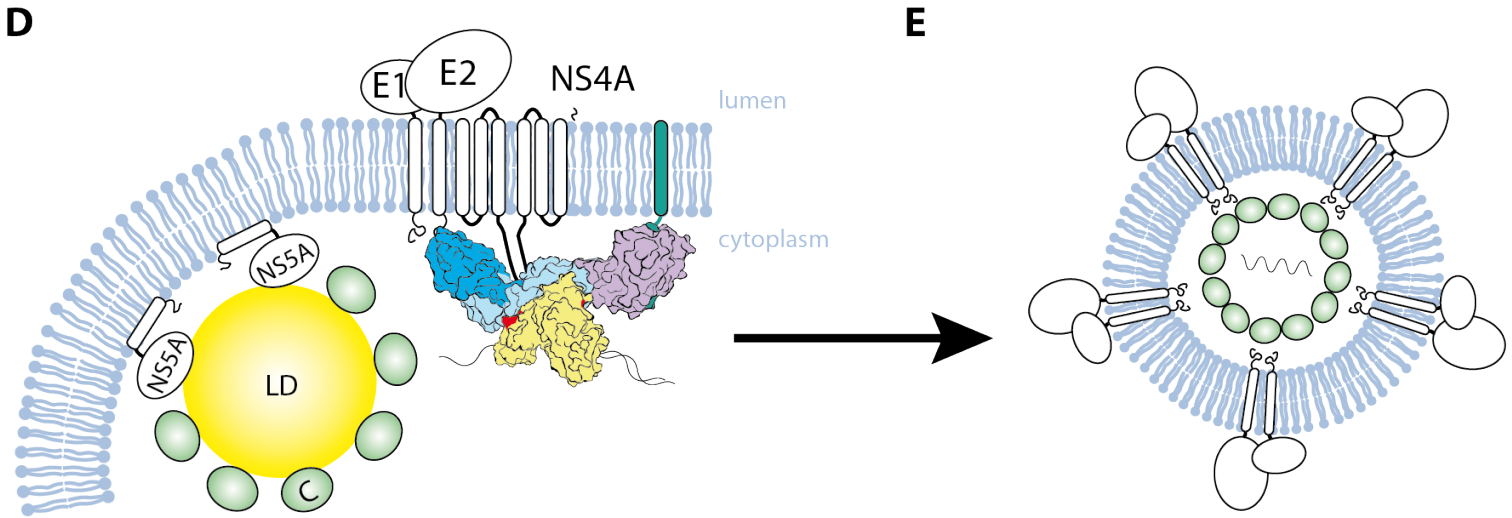

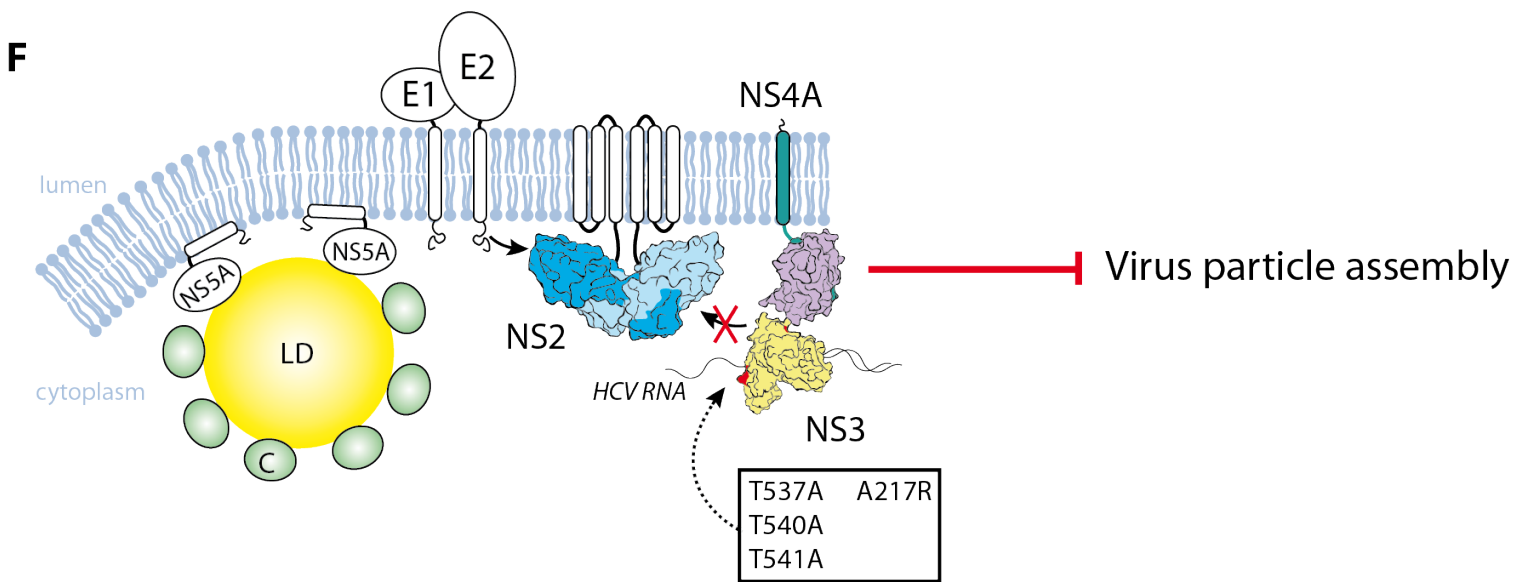

Figure 43 Model of HCV virus particle assembly. (A) Overview on virion assembly pathway within the infected cell. It is thought that early steps of particle assembly are located at ER derived membranes in close proximity to the rough ER and cytoplasmic lipid droplets. Further pathway is assumed to include binding of (pre-) VLDL or HDL particles and processing within the Golgi apparatus followed by exocytose. (B) Core- and NS5A-coated lipid droplets might initiate assembly inducing 
the formation of a NS2-coordinated complex. Within HCV infected cells core induced lipid droplet dynamics are described (Lyn RK et al. 2010). Furthermore, interactions might induce the formation of a complex between E1-E2 with NS2 and NS3. NS2 is shown in its dimeric form as recently described in the context of viral its catalytic activity within the NS2-3 protease. Monomers are colored blue and lightblue, transmembrane domains are shown in white. NS3 protease domain is colored purple, the helicase domain is colored yellow. NS4A is colored green. Viral RNA is simplified. Core, NS5A, E1-E2 and NS4A are shown schematically. NS2 and NS3 are shown as protein surface models. Protein models were made on the basis of 3D models rendered with PyMOL. Protein data bank entries $2 \mathrm{HDO}, 3 \mathrm{KQL}$ and $3 \mathrm{P} 8 \mathrm{~N}$ were used. Arrows indicate interactions between proteins as revealed here and by Stapleford et al. (2011). (C) Model of proposed complex of E1-E2/NS2/NS3. Results of this study indicate that NS2 interacts with NS3hel. Sites implicated in this interaction, T537, T540, H541 and A217, are highlighted red. (D) Formation of a virus particle. The NS2 complex coordinates interactions between E1-E2 and NS3. NS3hel might coordinate viral RNA to the site of assembly. Interactions between core and E1-E2 lead to encapsidation of viral RNA. (E) Virus particle. ER derived membranes including structural proteins are formed around the encapsidated viral genome. (F) Mutagenesis of residues T537, T540, H541 and A217 disrupt interactions with NS2 and thereby the stability of the complex. Mutations block virus particle assembly as interactions with E1-E2 are reduced and viral RNA is not introduced to assembly sites and is not encapsidated.

However, mutations T612A and H613A within NS3hel domain 3 do not significantly influence NS2-NS3 interactions but lead also to a defect in particle assembly. NS3 was captured in an equal level compared to WT genotype 2a construct. Mutations within JGN3-2 have to interfere somewhere else in the process of particle assembly. Surface residues T612 and H613 seem to influence assembly in a different way than residues T537, T540, H541 and A217. They might interact with other HCV proteins or host cell proteins or obtain other roles within the assembly pathway.

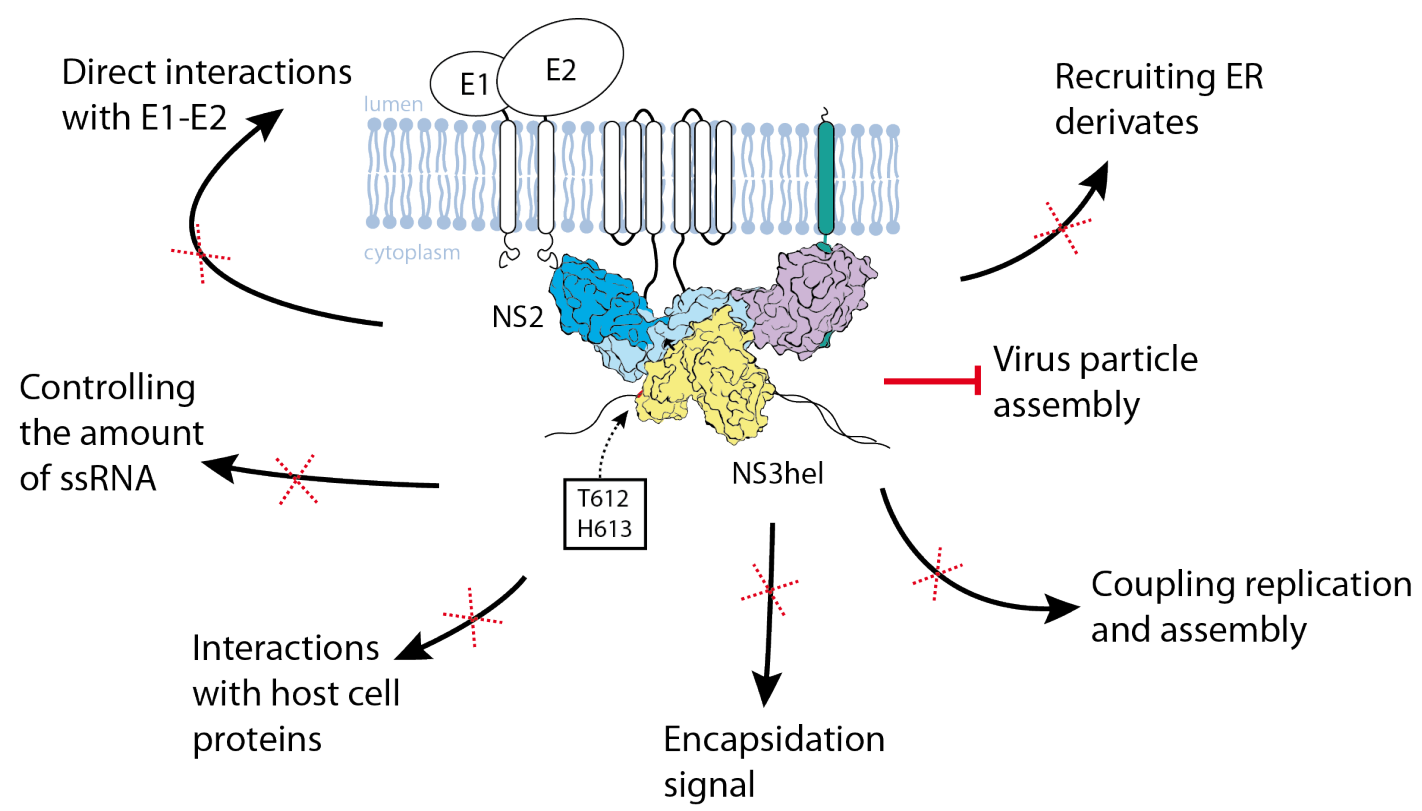

Figure 44 Suggested interactions of NS3hel during HCV particle assembly. The figure shows NS3 in complex with NS2. NS3hel residues T612 and $\mathrm{H} 613$ within domain 3 are highlighted red. Possible interactions are shown dashed crossed out arrows indicate suggested defects that might lead to a lack of particle assembly. Further descriptions are found in the text. 
Interactions between NS3 and so far unknown host cell proteins could facilitate genome packaging and virion assembly which could be influenced by surface residues of NS3 domain 1. The protein-protein affinity pulldown system could be further used to determine NS3hel interactions with host cell proteins.

Packaging pathway of the adenovirus type 2 and the rotavirus showed that its genome encapsidation is mediated by NTPase or helicase activity (King et al. 2001; Taraporewala and Patton 2004). However, as replication and thereby NTPase and helicase activities were intact, this role of NS3hel can be ruled out.

Moreover, NS3hel might be important for coupling RNA replication and packaging. Coupling between these functions is previously described for polioviruses (Nugent et al. 1999). As NS3 is involved in both viral replication and packaging it could function as a link between replication and assembly. Different authors suggested that within the poliovirus context only actively replicating RNA could be encapsidated (Baltimore 1969; Nugent et al. 1999). Khromykh et al. (2001) demonstrated a functional coupling between the replication and packaging of flavivirus RNA. These findings indicate that both complexes, the replication and packaging complex, have to be coupled somehow. A protein like NS3 that is involved in both, replication and assembly, is predestinated for the role of linking these functions and regulating particle assembly.

Suggesting that helicases are able to control the amount of sSRNA and regulate replication by their unwinding activities another possible role of NS3hel in viral assembly could be the regulation of virus assembly. By controlling the amount of single-stranded HCV genome NS3hel could be a limiting factor for particle assembly. If no single-stranded genome is available for packaging, assembly is blocked. However, controlling only assembly without replication seems to be unlikely. There might be other factors that stimulate NS3hel to act in assembly instead of replication and that are influenced by the inserted mutations.

In other RNA viruses, like alpha viruses, an encapsidation signal on the RNA level is required for specific genome packaging into infectious particles. A specific sequence is important for capsid recognition and initiates the formation of viral nucleocapsids. In flavivirus assembly such an encapsidation signal is not yet identified. As identified residues involved in virus assembly are well conserved they could possibly represent such a signal sequence. However, as residues are located on the surface of the completely folded protein it is more likely that they are involved in interactions on the protein level.

Dentzer et al. (2009) revealed several C-terminal NS2 residues that are required for the production of infectious virus particles. These residues did not influence replication or NS2 protease activity. Determined residues might be involved in protein-protein interactions with E1-E2 or NS3. Stapleford and Lindenbach (2011) revealed that NS2 might act as a coordinator between NS3 and E1-E2. Interactions between NS3 and E1-E2 were disrupted 
without active NS2. Based on these findings it seems unlikely that NS3 interacts directly with E1-E2. NS2 seems to bring together NS3 and E1-E2 to the sites of assembly. But afterwards NS3 and E1-E2 might interact directly and these direct interactions may be disrupted by introducing the changes to NS3hel. NS2-NS3 interactions would be intact but assembly would be blocked at the following step. These speculations have to be studied further.

Furthermore, the site of HCV replication was identified as ER derived membranous web. NS4B induces membrane alterations that provide a location for the replication complex. ER derived membrane alterations are also described in the context of virion assembly (Bartenschlager et al. 2004). Membrane alterations are thought to be induced by NS2 to provide sites for virion assembly alike to NS2A in other flaviviruses (Leung et al. 2008). If these membranous structures are recruited from the ER or from other origins is not described. As there is a possibility that replication and assembly are linked, the membranous web might be the origin of membrane structures for viral assembly sites. NS3 that functions in the RNA replication complex could possibly play a role in recruiting these membranes. NS3 could also play a role in recruiting ER derivates. But these speculations remain unknown so far.

\subsection{Long term infectivity}

Next to the assembly studies, the ability of HCV to restore defects in infectivity was confirmed. Therefore, transfected Huh7.5 cells were constantly passaged over a period of twelve days. All three mutants - JGN3-2, JGN3-12 and JGN3-24 - regained infectivity. JGN3-2 regained infectivity of an average of 6 days, JGN3-12 and JGN3-24 after nine days. HCV lacks RdRp proofreading activity, which leads to a high mutation rate. Mutations within the used mutants fixed the defect in virus assembly. New mutations could have been second site changes or reversions to WT.

As JGN3-2 is regaining infectivity after an average of six days, in contrast to above suggestions regarding virion assembly, residues T612 and H613 might only influence reaction kinetics within the assembly pathway resulting in a delay of virus particle production. A low amount of virions might be produced but not enough for infecting naïve cells. However, as replication is intact kinetics of the NS3 helicase enzyme activities cannot be affected which makes a problem with reaction kinetics unlikely. 


\subsection{Outlook}

Understanding the role of NS3hel in virus particle assembly helps to unravel the process of the formation of infectious virions. Understanding the role of HCV assembly has potential to help finding a way to block infectivity of the virus. Understanding this pathway can lead to discover novel ways to interfere HCV infectivity. Finding novel substances to treat hepatitis $\mathrm{C}$ or to find a vaccine against it depends on understanding every step of the HCV life cycle. Virus particle assembly and NS3 might be important targets for future drug developments as a mostly undiscovered part of the virus life cycle.

NS3hel could be shown to be essential for viral particle assembly. It is also shown to interact with NS2. To identify the process of HCV particle assembly and the role of NS3hel in this process further studies have to carry on the previous work. Interactions between NS3 and host cell proteins should be studied. Whether NS3hel functions as a coordinator of ssRNA for encapsidation or is just maintaining its unwinding activity has to be confirmed by future experiments. One major problem determining these interactions is that RNA binding cannot be blocked as replication has to be intact. Furthermore, whether NS3hel acts a linker between replication and assembly has to be studied deeper.

However, results presented here contribute in unraveling HCV particle assembly. Domains 1 and 3 could be demonstrated being essential for this process and protein-protein interactions could be proven being crucial for virion assembly. Domain 3 could be presented to be involved in this pathway for the first time. 


\section{Summary}

Hepatitis C virus non-structural protein 3 (NS3) is a multifunctional protein that obtains serine protease, RNA helicase and NTPase activities. The enzyme is essential for viral RNA replication. Its protease domain cleaves the viral polyprotein at multiple sites. Furthermore, NS3 has recently been implicated to play a role in virus particle assembly. But its function in the assembly process is unknown so far.

To determine the role of NS3 in HCV particle assembly alanine scanning mutagenesis within the NS3 helicase domain of chimerical genotype 2a full length replicons was performed. 25 mutants with changes of surface residues within the helicase domain were tested. 16 mutants showed moderate or severe defects in replication. 6 mutants were able to replicate and produce infectious virus as efficiently as wild type Jc1. 3 mutants efficiently replicated but had severe defects in virus particle assembly. Residues T612, H613 T537, T540, H541 and A217 within helicase subdomain 1 and 3 were identified being essential for HCV virion assembly. Domain 3 could be shown to be involved in particle assembly for the first time. Furthermore, RT-PCR and freeze-thawing experiments were used to determine the step within the particle assembly process affected by the NS3 helicase domain. The involvement of NS3 helicase domains 1 and 3 in virion assembly could be identified at an early step of the process prior to the formation of intracellular infectious particles. To determine interactions with NS2, protein complexes containing biotinylated NS2 were affinity captured with streptavidin magnetic beads. Previously described physically interactions between NS2 and NS3 were confirmed and 2 of the assembly defective NS3 helicase mutants were shown to influence these interactions but not completely disrupt NS2-NS3 protein complexes. The remaining mutant did not influence these interactions.

Data within this thesis reveal that NS3 helicase is involved in an early step of HCV particle assembly. Helicase domain 1 and 3 could be identified to be essential in this process. Furthermore, interactions with NS2 seem to play a central role in the assembly process. 


\section{Appendix}

\section{List of figures}

Figure 1 Phylogenetic tree of major HCV genotypes and subtypes........................................

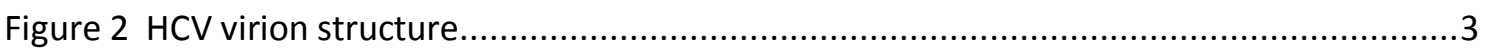

Figure $3 \mathrm{HCV}$ genome structure and polyprotein processing ............................................ 5

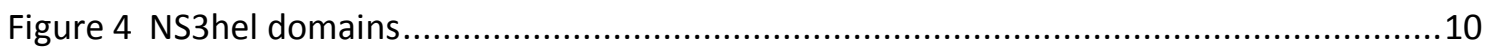

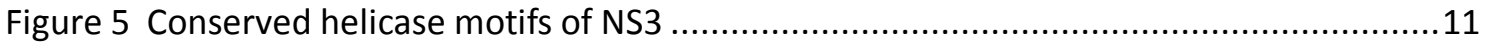

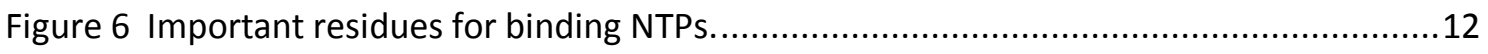

Figure 7 Model of NS3hel nucleic acid translocation and unwinding .....................................14

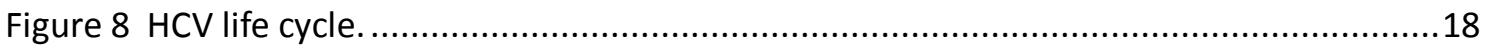

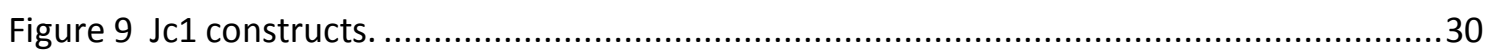

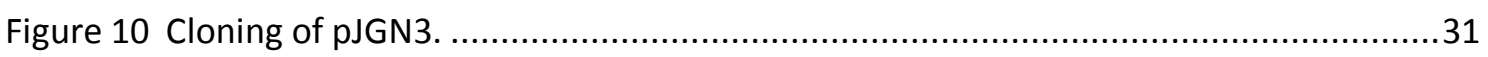

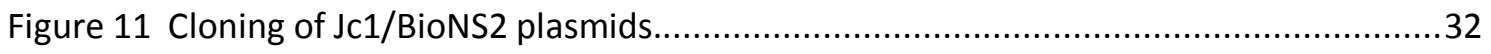

Figure 12 Huh 7.5 cells in different stages of growth after splitting......................................36

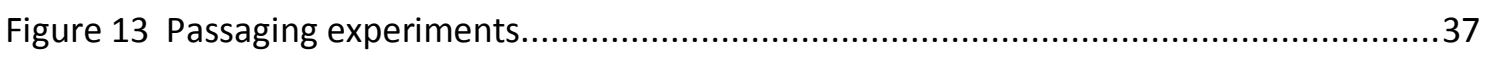

Figure 14 GLuc is a sensitive reporter for virus replication and infectivity.............................38

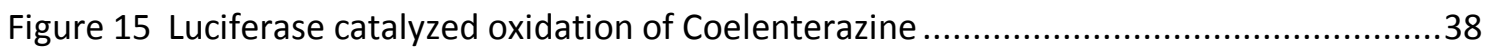

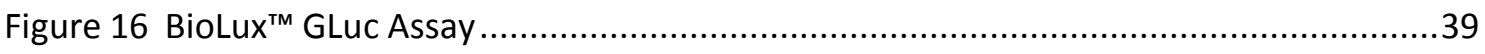

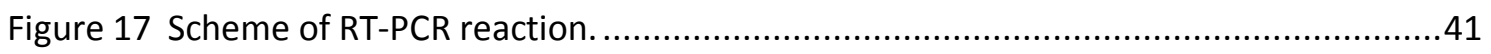

Figure 18 Biotinylation of the biotin acceptor peptide (BAP) catalysed by E.coli BirA ..............43

Figure 19 Pulldown of biotinylated proteins using Streptavidin magnetic beads .....................45

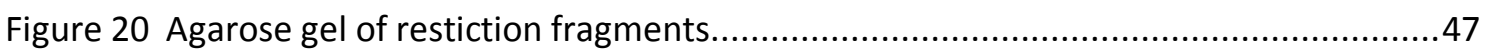

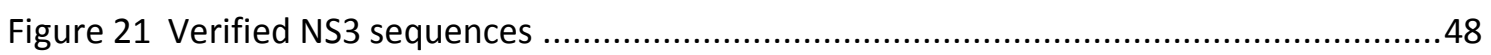

Figure 22 Locations of mutations within NS3hel domain 3 ..............................................49

Figure 23 Replication of JGN3 constructs with changes in $\alpha$-helices 20 and 21 of dom3..........52

Figure 24 Replication of JGN3 constructs with changes in $\alpha$-helices 14,15 and 16 of dom3 ....53

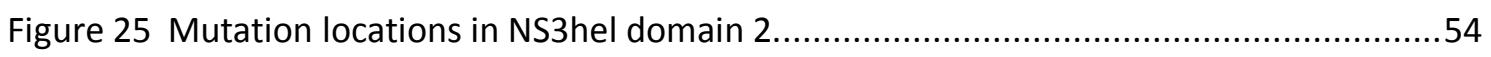

Figure 26 Replication of JGN3 constructs with changes in domain 2 ..................................56

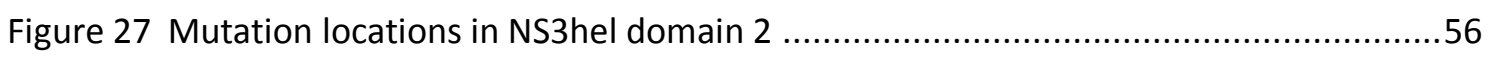

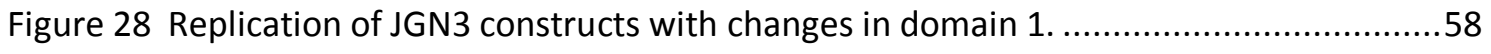

Figure 29 Infectivity results of JGN3 constructs with changes within domain 3 alpha

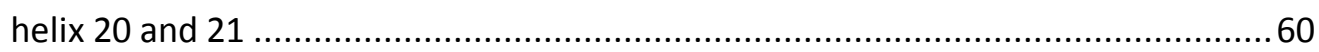

Figure 30 Infectivity of JGN3 constructs with changes within domain 3 alpha helix

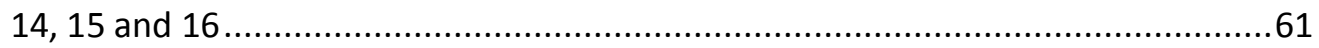

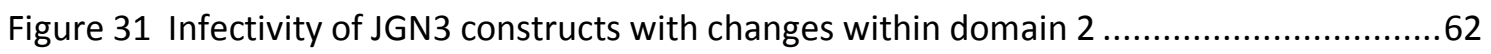

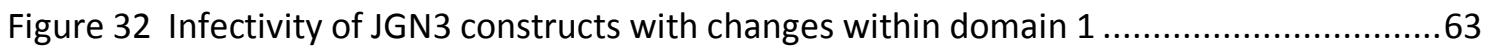

Figure 33 Summary of replication and infectivity experiments and NS5a immunostaining

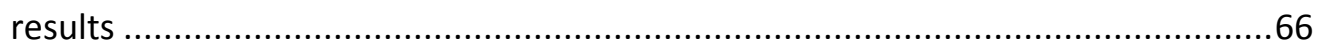

Figure 34 JGN3-2, JGN3-12 and JGN3-24 - mutations, replication and infectivity ..................67

Figure 35 RNA release of infectivity-defective NS3hel mutants..........................................68 
Figure 36 Intracellular infectivity vs. extracellular infectivity of NS3hel mutants ....................70

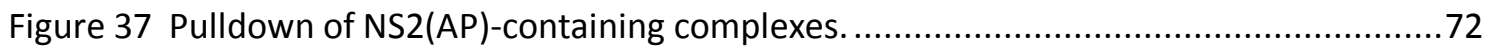

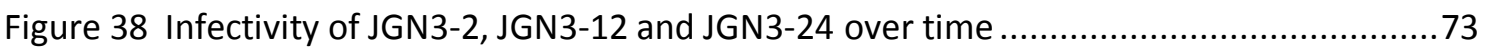

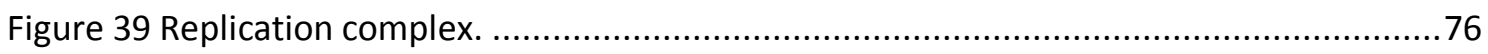

Figure 40 Involvement of studied domain 3 residues in replication.........................................77

Figure 41 Involvement of studied domain 1 and 2 residues in replications. ..........................79

Figure $42 \mathrm{NS} 3 \mathrm{hel}$ is essential for an early step of particle assembly prior to the formation

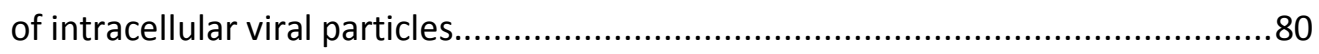

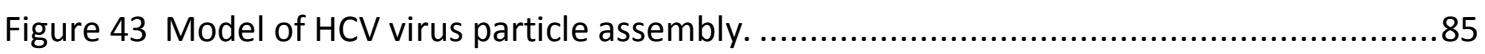

Figure 44 Suggested interactions of NS3hel during HCV particle assembly..............................86

References of figures are shown in the title below each figure. Figures without references are own pictures or photographs made with PyMOL, GraphPad Prism 5 and Adobe Illustrator.

\section{List of tables}

Table 1 Structural and non-structural HCV proteins. ...........................................................

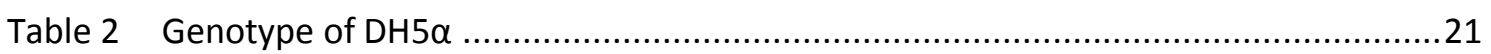

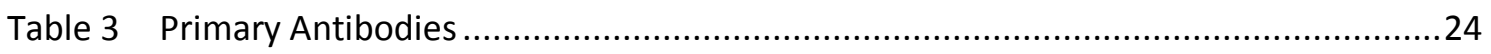

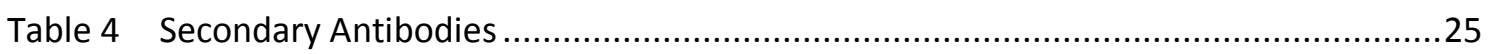

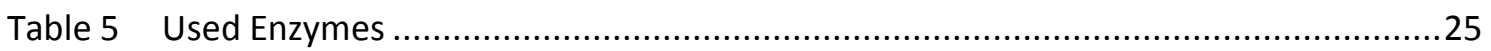

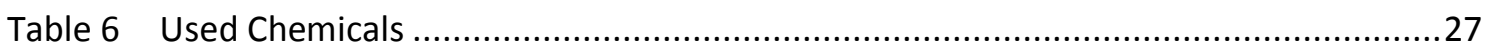

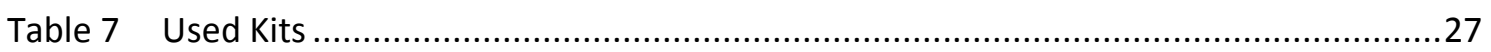

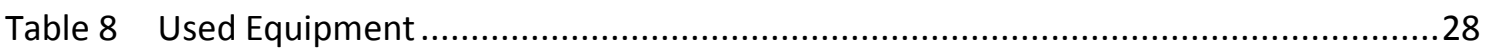

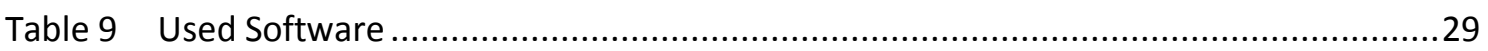

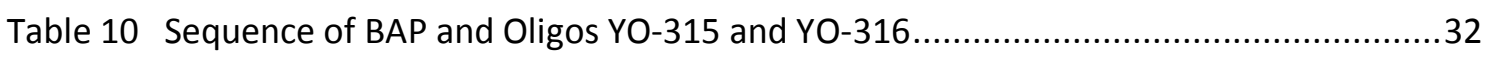

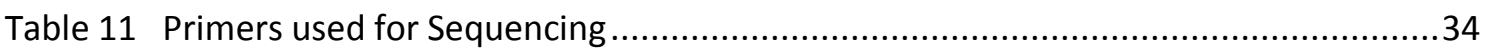

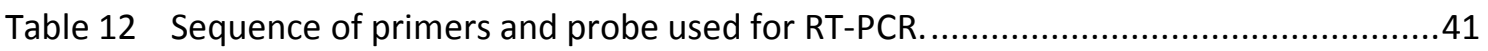

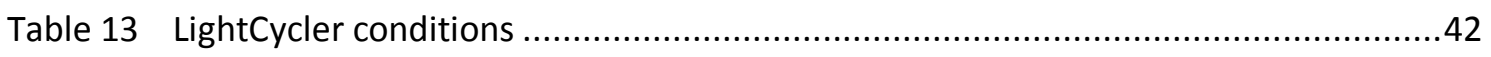

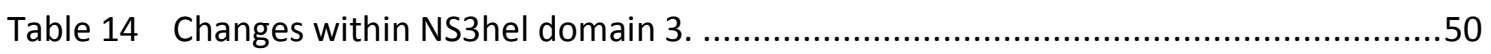

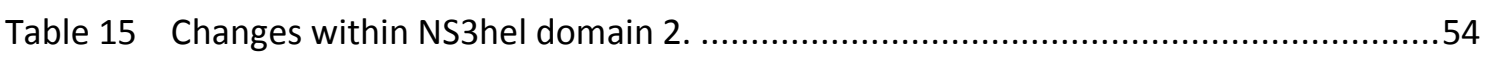

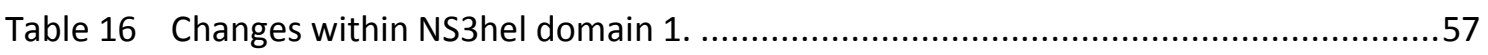




\section{References}

Agapov, E V; Murray, C L; Frolov, I; Qu, L; Myers, T M; Rice, C M (2004): Uncleaved NS2-3 is required for production of infectious bovine viral diarrhea virus. J Virol $\underline{78}$ (5), 2414-2425.

Baltimore, D: The replication of picomaviruses. In: Levy, H B (Eds.) The biochemistry of viruses. Dekker, New York-London 1969, 101-176.

Barbato, G; Cicero, D O; Nardi, M C; Steinkuhler, C; Cortese, R; Francesco, R de; Bazzo, R (1999): The solution structure of the $\mathrm{N}$-terminal proteinase domain of the hepatitis $\mathrm{C}$ virus (HCV) NS3 protein provides new insights into its activation and catalytic mechanism. J Mol Biol $\underline{289}$ (2), 371-384.

Bartenschlager R; Frese M; Pietschmann T (2004): Novel insights into hepatitis C virus replication and persistence. Adv Virus Res $\underline{63}, 71-180$.

Bartosch, B; Cosset, F-L (2006): Cell entry of hepatitis C virus. Virology $\underline{348}$ (1), 1-12.

Blight, K J; Kolykhalov, A A; Rice, C M (2000): Efficient initiation of HCV RNA replication in cell culture. Science 290 (5498), 1972-1974.

Blight, K J; McKeating, J A; Rice, C M (2002): Highly permissive cell lines for subgenomic and genomic hepatitis C virus RNA replication. J Virol 76 (24), 13001-13014.

Boulant S; Targett-Adams P; McLauchlan J (2007): Disrupting the association of hepatitis C virus core protein with lipid droplets correlates with a loss in production of infectious virus. J Gen Virol $\underline{88}$ (8), 2204-2213.

Bradley, D; McCaustland, K; Krawczynski, K; Spelbring, J; Humphrey, C; Cook, E H (1991): Hepatitis C virus: buoyant density of the factor VIII-derived isolate in sucrose. J Med Virol 34 (3), 206-208.

Carrere-Kremer, S; Montpellier-Pala, C; Cocquerel, L; Wychowski, C; Penin, F; Dubuisson, J (2002): Subcellular Localization and Topology of the p7 Polypeptide of Hepatitis C Virus. J Virol 76 (8), 3720-3730.

Choo, Q L; Richman, K H; Han, J H; Berger, K; Lee, C; Dong, C; Gallegos, C; Coit, D; Medina-Selby, R; Barr, P J (1991): Genetic organization and diversity of the hepatitis C virus. Proc Natl Acad Sci USA 88 (6), 2451-2455.

Cocquerel, L; Voisset, C; Dubuisson, J (2006): Hepatitis C virus entry: potential receptors and their biological functions. J Gen Virol $\underline{87}$ (5), 1075-1084.

Cormier, E G; Tsamis, F; Kajumo, F; Durso, R J; Gardner, J P; Dragic, T (2004): CD81 is an entry coreceptor for hepatitis C virus. Proc Natl Acad Sci USA 101 (19), 7270-7274.

De Francesco, R; Urbani, A; Nardi, M C; Tomei, L; Steinkühler, C; Tramontano, A (1996): A Zinc Binding Site in Viral Serine Proteinases. Biochemistry 35 (41), 13282-13287.

Dentzer, T G; Lorenz, I C; Evans, M J; Rice, C M (2009): Determinants of the hepatitis C virus nonstructural protein 2 protease domain required for production of infectious virus. $J$ Virol 83 (24), 12702-12713. 
Dillingham, M S; Soultanas, P; Wiley, P; Webb, M R; Wigley, D B (2001): Defining the roles of individual residues in the single-stranded DNA binding site of PcrA helicase. Proc Natl Acad Sci USA $\underline{98}$ (15), 8381-8387.

Dubuisson, J (2007): Hepatitis C virus proteins. World J Gastroenterol 13 (17), 2406-2415.

Egger, D; Wölk, B; Gosert, R; Bianchi, L; Blum, H E; Moradpour, D; Bienz, K (2002): Expression of hepatitis $C$ virus proteins induces distinct membrane alterations including a candidate viral replication complex. J Virol $\underline{76}$ (12), 5974-5984.

Einav, S; Elazar, M; Danieli, T; Glenn, J S (2004): A nucleotide binding motif in hepatitis C virus (HCV) NS4B mediates HCV RNA replication. J Virol $\underline{78}$ (20), 11288-11295.

Evans, M J; Rice, C M; Goff, S P (2004): Phosphorylation of hepatitis C virus nonstructural protein $5 \mathrm{~A}$ modulates its protein interactions and viral RNA replication. Proc Natl Acad Sci USA 101 (35), 13038-13043.

Friebe, P; Lohmann, V; Krieger, N; Bartenschlager, R (2001): Sequences in the 5' Nontranslated Region of Hepatitis C Virus Required for RNA Replication. J Virol 75 (24), 12047-12057.

Gastaminza, P; Kapadia, S B; Chisari, F V (2006): Differential biophysical properties of infectious intracellular and secreted hepatitis C virus particles. J Virol $\underline{80}$ (22), 11074-11081.

Gorbalenya, A; Koonin, E (1993): Helicases: amino acid sequence comparisons and structure-function relationships. Curr Opin Struct Biol $\underline{3}, 419-429$.

Gosert, R; Egger, D; Lohmann, V; Bartenschlager, R; Blum, H E; Bienz, K; Moradpour, D (2003): Identification of the hepatitis C virus RNA replication complex in Huh-7 cells harboring subgenomic replicons. J Virol $\underline{77}$ (9), 5487-5492.

Griffin, S D C; Beales, L P; Clarke, D S; Worsfold, O; Evans, S D; Jaeger, J; Harris, M P G; Rowlands, D J (2003): The p7 protein of hepatitis $C$ virus forms an ion channel that is blocked by the antiviral drug, Amantadine. FEBS Lett $\underline{535}$ (1-3), 34-38.

Gu, B; Pruss, C M; Gates, A T; Khandekar, S S (2005): The RNA-unwinding activity of hepatitis $C$ virus non-structural protein 3 (NS3) is positively modulated by its protease domain. Protein Pept Lett 12 (4), 315-321.

Gu, M; Rice, C M (2010): Inaugural Article: Three conformational snapshots of the hepatitis $C$ virus NS3 helicase reveal a ratchet translocation mechanism. Proc Natl Acad Sci USA 107 (2), 521-528.

He, L F; Alling, D; Popkin, T; Shapiro, M; Alter, H J; Purcell, R H (1987): Determining the size of non-A, non-B hepatitis virus by filtration. J Infect Dis 156 (4), 636-640.

Hijikata, M; Shimizu, Y K; Kato, H; Iwamoto, A; Shih, J W; Alter, H J; Purcell, R H; Yoshikura, $H$ (1993): Equilibrium centrifugation studies of hepatitis $C$ virus: evidence for circulating immune complexes. J Virol $\underline{67}$ (4), 1953-1958.

Honda, M; Ping, L H; Rijnbrand, R C; Amphlett, E; Clarke, B; Rowlands, D; Lemon, S M (1996): Structural requirements for initiation of translation by internal ribosome entry within genome-length hepatitis C virus RNA. Virology 222 (1), 31-42. 
Ishido, S; Fujita, T; Hotta, H (1998): Complex formation of NS5B with NS3 and NS4A proteins of hepatitis C virus. Biochem Biophys Res Commun 244 (1), 35-40.

Ivashkina, N; Wölk, B; Lohmann, V; Bartenschlager, R; Blum, H E; Penin, F; Moradpour, D (2002): The hepatitis $C$ virus RNA-dependent RNA polymerase membrane insertion sequence is a transmembrane segment. J Virol $\underline{76}$ (24), 13088-13093.

Jankowsky, E (2010): RNA Helicase database. With assistance of Anja Jankowski et al. Available online at www.mahelicase.org, checked on 16/11/2011.

Jankowsky, E; Fairman, M E (2007): RNA helicases--one fold for many functions. Curr Opin Struct Biol 17 (3), 316-324.

Jirasko, V; Montserret, R; Lee, J Y; Gouttenoire, J; Moradpour, D; Penin, F; Bartenschlager, $R$ (2010): Structural and functional studies of nonstructural protein 2 of the hepatitis $C$ virus reveal its key role as organizer of virion assembly. PLoS Pathog $\underline{6}$ (12), e1001233.

Jones C T; Murray CL; Eastman DK; Tassello J; Rice CM (2007): Hepatitis C virus p7 and NS2 proteins are essential for production of infectious virus. J Virol 81 (16), 8374-8383.

Jones, D M; McLauchlan, J (2010): Hepatitis C virus: assembly and release of virus particles. J Biol Chem $\underline{285}$ (30), 22733-22739.

Kadaré, G; Haenni, A L (1997): Virus-encoded RNA helicases. J Virol 71 (4), 2583-2590.

Kato, T; Date, T; Miyamoto, M; Furusaka, A; Tokushige, K; Mizokami, M; Wakita, T (2003): Efficient replication of the genotype $2 a$ hepatitis $C$ virus subgenomic replicon. Gastroenterology $\underline{125}$ (6), 1808-1817.

Khromykh, A A; Varnavski, A N; Sedlak, P L; Westaway, E G (2001): Coupling between replication and packaging of flavivirus RNA: evidence derived from the use of DNA-based full-length cDNA clones of Kunjin virus. J Virol 75 (10), 4633-4640.

Kiiver, K; Merits, A; Ustav, M; Zusinaite, E (2006): Complex formation between hepatitis C virus NS2 and NS3 proteins. Virus Res 117 (2), 264-272.

Kim, J L; Morgenstern, K A; Griffith, J P; Dwyer, M D; Thomson, J A; Murcko, M A; Lin, C; Caron, P R (1998): Hepatitis C virus NS3 RNA helicase domain with a bound oligonucleotide: the crystal structure provides insights into the mode of unwinding. Structure $\underline{6}(1), 89-100$.

Kim, Y K; Lee, S H; Kim, C S; Seol, S K; Jang, S K (2003): Long-range RNA-RNA interaction between the 5 ' nontranslated region and the core-coding sequences of hepatitis $C$ virus modulates the IRES-dependent translation. RNA $\underline{9}$ (5), 599-606.

King, J A; Dubielzig, R; Grimm, D; Kleinschmidt, J A (2001): DNA helicase-mediated packaging of adeno-associated virus type 2 genomes into preformed capsids. EMBO J 20 (12), 3282-3291.

Koch, J O; Bartenschlager, R (1999): Modulation of hepatitis C virus NS5A hyperphosphorylation by nonstructural proteins NS3, NS4A, and NS4B. J Virol $\underline{73}$ (9), 71387146. 
Kolykhalov, A A; Mihalik, K; Feinstone, S M; Rice, C M (2000): Hepatitis C virus-encoded enzymatic activities and conserved RNA elements in the 3 ' nontranslated region are essential for virus replication in vivo. J Virol $\underline{74}$ (4), 2046-2051.

Krekulová, L; Rehák, V; Riley, L W (2006): Structure and functions of hepatitis C virus proteins: 15 years after. Folia Microbiol $\underline{51}$ (6), 665-680.

Kümmerer, B M; Rice, C M (2002): Mutations in the yellow fever virus nonstructural protein NS2A selectively block production of infectious particles. J Virol $\underline{76}$ (10), 4773-4784.

Lam, A M I; Frick, D N (2006): Hepatitis C virus subgenomic replicon requires an active NS3 RNA helicase. J Virol $\underline{80}$ (1), 404-411.

Lam AM; Keeney D; Frick DN (2003): Two novel conserved motifs in the hepatitis C virus NS3 protein critical for helicase action. J Biol Chem 278 (45), 44514-44524.

Lam, A M I; Rypma, R S; Frick, D N (2004): Enhanced nucleic acid binding to ATP-bound hepatitis $\mathrm{C}$ virus NS3 helicase at low pH activates RNA unwinding. Nucleic Acids Res $\underline{32}$ (13), 4060-4070.

Lemon, S M; Walker, C; Alter, M J; MinKyung, Y: Hepatitis C Virus. In: Knipe, David Mahan; Howley, Peter M. (Eds.): Fields' virology. Lippincott Williams \& Wilkins, Philadelphia 2007, 1253-1305.

Lesburg, C A; Cable, M B; Ferrari, E; Hong, Z; Mannarino, A F; Weber, P C (1999): Crystal structure of the RNA-dependent RNA polymerase from hepatitis $C$ virus reveals a fully encircled active site. Nat Struct Biol $\underline{6}$ (10), 937-943.

Leung JY; Pijlman GP; Kondratieva N; Hyde J; Mackenzie JM; Khromykh AA (2008): Role of nonstructural protein NS2A in flavivirus assembly. J Virol $\underline{82}$ (10), 4731-4741.

Li, K; Foy, E; Ferreon, J C; Nakamura, M; Ferreon, A C M; Ikeda, M; Ray, S C; Gale, M; Lemon, S M (2005): Immune evasion by hepatitis C virus NS3/4A protease-mediated cleavage of the Toll-like receptor 3 adaptor protein TRIF. Proc Natl Acad Sci USA 102 (8), 2992-2997.

Lin, C; Kim, J L (1999): Structure-based mutagenesis study of hepatitis C virus NS3 helicase. J Virol $\underline{73}$ (10), 8798-8807.

Lindenbach, B D; Rice, C M (2005): Unravelling hepatitis C virus replication from genome to function. Nature $\underline{436}$ (7053), 933-938.

Lindenbach, B D; Thiel, H-J; Rice, C M: Flaviviridae: The Viruses and Their Replication. In: Knipe, David Mahan; Howley, Peter M. (Eds.): Fields' virology. Lippincott Williams \& Wilkins, Philadelphia 2007, 1101-1152.

Liu, W J; Sedlak, P L; Kondratieva, N; Khromykh, A A (2002): Complementation analysis of the flavivirus Kunjin NS3 and NS5 proteins defines the minimal regions essential for formation of a replication complex and shows a requirement of NS3 in cis for virus assembly. J Virol $\underline{76}$ (21), 10766-10775.

Lo, S Y; Masiarz, F; Hwang, S B; Lai, M M; Ou, J H (1995): Differential subcellular localization of hepatitis $C$ virus core gene products. Virology 213 (2), 455-461. 
Lohmann, V; Körner, F; Koch, J; Herian, U; Theilmann, L; Bartenschlager, R (1999): Replication of subgenomic hepatitis $\mathrm{C}$ virus RNAs in a hepatoma cell line. Science $\underline{\mathbf{2 8 5}}$ (5424), 110-113.

Lorenz, I C (2010): The Hepatitis C Virus Nonstructural Protein 2 (NS2): An Up-and-Coming Antiviral Drug Target. Viruses 2 (8), 1635-1646.

Lyn RK; Kennedy DC; Stolow A; Ridsdale A; Pezacki JP (2010): Dynamics of lipid droplets induced by the hepatitis C virus core protein. Biochem Biophys Res Commun 399 (4), 518524.

Ma, Y; Yates, J; Liang Y; Lemon, S M; Yi, M (2008): NS3 helicase domains involved in infectious intracellular hepatitis C virus particle assembly. J Virol $\underline{82}$ (15), 7624-7639.

Ma, Y; Anantpadma, M; Timpe, J M; Shanmugam, S; Singh, S M; Lemon, S M; Yi, M (2011): Hepatitis $C$ virus NS2 protein serves as a scaffold for virus assembly by interacting with both structural and nonstructural proteins. J Virol $\underline{85}$ (1), 86-97.

Manns, M P; McHutchison, J G; Gordon, S C; Rustgi, V K; Shiffman, M; Reindollar, R; Goodman, Z D; Koury, K; Ling, M; Albrecht, J K (2001): Peginterferon alfa-2b plus ribavirin compared with interferon alfa-2b plus ribavirin for initial treatment of chronic hepatitis $\mathrm{C}: \mathrm{a}$ randomised trial. Lancet 358 (9286), 958-965.

McLauchlan, J (2000): Properties of the hepatitis C virus core protein: a structural protein that modulates cellular processes. J Viral Hepat $\underline{7}$ (1), 2-14.

Merola, M; Brazzoli, M; Cocchiarella, F; Heile, J M; Helenius, A; Weiner, A J; Houghton, M; Abrignani, S (2001): Folding of hepatitis C virus E1 glycoprotein in a cell-free system. J Virol $\underline{75}$ (22), 11205-11217.

Miyanari, Y; Atsuzawa, K; Usuda, N; Watashi, K; Hishiki, T; Zayas, M; Bartenschlager, R; Wakita, T; Hijikata, M; Shimotohno, K (2007): The lipid droplet is an important organelle for hepatitis C virus production. Nat Cell Biol $\underline{9}$ (9), 1089-1097.

Moradpour, D; Evans, M J; Gosert, R; Yuan, Z; Blum, H E; Goff, S P; Lindenbach, B D; Rice, C M (2004): Insertion of green fluorescent protein into nonstructural protein 5A allows direct visualization of functional hepatitis C virus replication complexes. J Virol $\underline{78}$ (14), 7400-7409.

New England Biolabs (2011): Manual Gaussia Luciferase Kit E3300S. Available online at http://www.neb.com/nebecomm/ManualFiles/manualE3300.pdf, updated on 07/2011, checked on $21 / 10 / 2011$.

Nugent, C I; Johnson, K L; Sarnow, P; Kirkegaard, K (1999): Functional coupling between replication and packaging of poliovirus replicon RNA. J Virol $\underline{73}$ (1), 427-435.

Owsianka, A M; Patel, A H (1999): Hepatitis C virus core protein interacts with a human DEAD box protein DDX3. Virology 257 (2), 330-340.

Pawlotsky, J-M (2003): Hepatitis C virus genetic variability: pathogenic and clinical implications. Clin Liver Dis $\underline{7}(1), 45-66$.

Phan, T; Beran, R K F; Peters, C; Lorenz, I C; Lindenbach, B D (2009): Hepatitis C virus NS2 protein contributes to virus particle assembly via opposing epistatic interactions with the E1E2 glycoprotein and NS3-NS4A enzyme complexes. J Virol $\underline{83}$ (17), 8379-8395. 
Phan, T; Kohlway, A; Dimberu, P; Pyle, A M; Lindenbach, B D (2011): The acidic domain of hepatitis $\mathrm{C}$ virus NS4A contributes to RNA replication and virus particle assembly. J Virol $\underline{85}$ (3), 1193-1204.

Piccininni, S; Varaklioti, A; Nardelli, M; Dave, B; Raney, K D; McCarthy, J E G (2002): Modulation of the hepatitis $C$ virus RNA-dependent RNA polymerase activity by the nonstructural (NS) 3 helicase and the NS4B membrane protein. J Biol Chem $\underline{277}$ (47), 4567045679 .

Pietschmann, T; Kaul, A; Koutsoudakis, G; Shavinskaya, A; Kallis, S; Steinmann, E; Abid, K; Negro, F; Dreux, M; Cosset, F-L; Bartenschlager, R (2006): Construction and characterization of infectious intragenotypic and intergenotypic hepatitis $\mathrm{C}$ virus chimeras. Proc Natl Acad Sci USA 103 (19), 7408-7413.

Pileri, P; Uematsu, Y; Campagnoli, S; Galli, G; Falugi, F; Petracca, R; Weiner, A J; Houghton, M; Rosa, D; Grandi, G; Abrignani, S (1998): Binding of hepatitis C virus to CD81. Science 282 (5390), 938-941.

Preugschat, F; Danger, D P; Carter, L H; Davis, R G; Porter, D J (2000): Kinetic analysis of the effects of mutagenesis of W501 and V432 of the hepatitis C virus NS3 helicase domain on ATPase and strand-separating activity. Biochemistry 17 . 5174-5183.

Raney, K D; Sharma, S D; Moustafa, I M; Cameron, C E (2010): Hepatitis C Virus Nonstructural Protein 3 (HCV NS3): A Multifunctional Antiviral Target. J Biol Chem $\underline{285}$ (30), 22725-22731.

Reynolds, J E; Kaminski, A; Kettinen, H J; Grace, K; Clarke, B E; Carroll, A R; Rowlands, D $\mathrm{J}$; Jackson, R J (1995): Unique features of internal initiation of hepatitis C virus RNA translation. EMBO J 14 (23), 6010-6020.

Saikrishnan, K; Griffiths, S P; Cook, N; Court, R; Wigley, D B (2008): DNA binding to RecD: role of the 1B domain in SF1B helicase activity. EMBO J $\underline{27}$ (16), 2222-2229.

Sakai, A; Claire, M S; Faulk, K; Govindarajan, S; Emerson, S U; Purcell, R H; Bukh, J (2003): The $p 7$ polypeptide of hepatitis $C$ virus is critical for infectivity and contains functionally important genotype-specific sequences. Proc Natl Acad Sci USA 100 (20), 11646-11651.

Scarselli, E; Ansuini, H; Cerino, R; Roccasecca, R M; Acali, S; Filocamo, G; Traboni, C; Nicosia, A; Cortese, R; Vitelli, A (2002): The human scavenger receptor class B type I is a novel candidate receptor for the hepatitis C virus. EMBO J 21 (19), 5017-5025.

Schregel, V; Jacobi, S; Penin, F; Tautz, N (2009): Hepatitis C virus NS2 is a protease stimulated by cofactor domains in NS3. Proc Natl Acad Sci USA 106 (13), 5342-5347.

Shi, S T; Lai, M M: HCV 5' and 3'UTR: When Translation Meets Replication. In: Tan, SengLai (Eds.): Hepatitis C Viruses: Genomes and Molecular Biology. Horizon Bioscience, Norfolk, UK 2006, 49-89.

Simmonds, P (2004): Genetic diversity and evolution of hepatitis C virus--15 years on. J Gen Virol $\underline{85}$ (Pt 11), 3173-3188.

Spahn, C M; Kieft, J S; Grassucci, R A; Penczek, P A; Zhou, K; Doudna, J A; Frank, J (2001): Hepatitis $C$ virus IRES RNA-induced changes in the conformation of the 40s ribosomal subunit. Science 291 (5510), 1959-1962. 
Stapleford, K A; Lindenbach, B D (2011): Hepatitis C virus NS2 coordinates virus particle assembly through physical interactions with the E1-E2 glycoprotein and NS3-NS4A enzyme complexes. J Virol $\underline{85}$ (4), 1706-1717.

Steinmann, E; Penin, F; Kallis, S; Patel, A H; Bartenschlager, R; Pietschmann, T (2007): Hepatitis $C$ virus $p 7$ protein is crucial for assembly and release of infectious virions. PLoS Pathog $\underline{3}(7)$, e103.

Suzich, J A; Tamura, J K; Palmer-Hill, F; Warrener, P; Grakoui, A; Rice, C M; Feinstone, S M; Collett, M S (1993): Hepatitis C virus NS3 protein polynucleotide-stimulated nucleoside triphosphatase and comparison with the related pestivirus and flavivirus enzymes. J Virol $\underline{67}$ (10), 6152-6158.

Tai, C L; Chi, W K; Chen, D S; Hwang, L H (1996): The helicase activity associated with hepatitis $\mathrm{C}$ virus nonstructural protein 3 (NS3). J Virol $\underline{70}$ (12), 8477-8484.

Tai C L; Pan W C; Liaw SH; Yang UC; Hwang LH; Chen DS (2001): Structure-based mutational analysis of the hepatitis C virus NS3 helicase. J Virol $\underline{75}$ (17), 8289-8297.

Tannous, B A; Kim, D-E; Fernandez, J L; Weissleder, R; Breakefield, X O (2005): Codonoptimized Gaussia luciferase cDNA for mammalian gene expression in culture and in vivo. Mol Ther 11 (3), 435-443.

Taraporewala, Z F; Patton, J T (2004): Nonstructural proteins involved in genome packaging and replication of rotaviruses and other members of the Reoviridae. Virus Res 101 (1), 5766.

Tellinghuisen, T L; Marcotrigiano, J; Rice, C M (2005): Structure of the zinc-binding domain of an essential component of the hepatitis C virus replicase. Nature 435 (7040), 374-379.

Tellinghuisen, T L; Foss, K L; Treadaway, J (2008): Regulation of hepatitis C virion production via phosphorylation of the NS5A protein. PLoS Pathog 4 (3), e1000032.

Tsukiyama-Kohara, K; lizuka, N; Kohara, M; Nomoto, A (1992): Internal ribosome entry site within hepatitis C virus RNA. J Virol $\underline{66}$ (3), 1476-1483.

Wakita, T; Pietschmann, T; Kato, T; Date, T; Miyamoto, M; Zhao, Z; Murthy, K; Habermann, A; Kräusslich, H-G; Mizokami, M; Bartenschlager, R; Liang, T J (2005): Production of infectious hepatitis $C$ virus in tissue culture from a cloned viral genome. Nat Med 11 (7), 791796.

Walker, J E; Saraste, M; Runswick, M J; Gay, N J (1982): Distantly related sequences in the alpha- and beta-subunits of ATP synthase, myosin, kinases and other ATP-requiring enzymes and a common nucleotide binding fold. EMBO J 1 (8), 945-951.

Wang, C; Sarnow, P; Siddiqui, A (1993): Translation of human hepatitis C virus RNA in cultured cells is mediated by an internal ribosome-binding mechanism. J Virol $\underline{67}$ (6), 33383344.

Welbourn, S; Pause, A (2007): The hepatitis C virus NS2/3 protease. Curr Issues Mol Biol $\underline{9}$ (1), 63-69.

Wiles, S; Ferguson, K; Stefanidou, M; Young, D B; Robertson, B D (2005): Alternative luciferase for monitoring bacterial cells under adverse conditions. Appl Environ Microbiol 71 (7), 3427-3432. 
Wölk, B; Sansonno, D; Kräusslich, H G; Dammacco, F; Rice, C M; Blum, H E; Moradpour, D (2000): Subcellular localization, stability, and trans-cleavage competence of the hepatitis $C$ virus NS3-NS4A complex expressed in tetracycline-regulated cell lines. J Virol $\underline{74}$ (5), 22932304.

$\mathrm{Xu}, \mathrm{Z}$ (2001): Synthesis of a novel hepatitis $\mathrm{C}$ virus protein by ribosomal frameshift. The EMBO 20 (14), 3840-3848.

Yamaga, A K; Ou, J H (2002): Membrane topology of the hepatitis C virus NS2 protein. J Biol Chem 277 (36), 33228-33234.

Yao, N; Hesson, T; Cable, M; Hong, Z; Kwong, A; Le, H; Weber, P C (1997): Structure of the hepatitis $\mathrm{C}$ virus RNA helicase domain. Nat Struct Mol Biol 4 (6), 463-467.

Yi, M; Lemon, S M (2003): 3' Nontranslated RNA Signals Required for Replication of Hepatitis C Virus RNA. J Virol $\underline{77}$ (6), 3557-3568.

You, S; Rice, C M (2008): 3' RNA elements in hepatitis C virus replication: kissing partners and long poly(U). J Virol $\underline{82}$ (1), 184-195.

Yuasa, T; Ishikawa, G; Manabe, S; Sekiguchi, S; Takeuchi, K; Miyamura, T (1991): The particle size of hepatitis $C$ virus estimated by filtration through microporous regenerated cellulose fibre. J Gen Virol $\underline{72}$ (8)2021-2024.

Zhang, C; Cai, Z; Kim, Y-C; Kumar, R; Yuan, F; Shi, P-Y; Kao, C; Luo, G (2005): Stimulation of hepatitis $\mathrm{C}$ virus (HCV) nonstructural protein 3 (NS3) helicase activity by the NS3 protease domain and by HCV RNA-dependent RNA polymerase. J Virol $\underline{79}$ (14), 8687-8697. 


\section{Acknowledgements}

I would like to thank my thesis advisor, Professor Frank Hufert, for his support and encouragement regarding my plans for this project.

I am most grateful to Brett Lindenbach from the Yale University for giving me the unique opportunity carrying out my doctoral thesis in his laboratory and working on this interesting project. I am heartily thankful for his constant guidance and support during the project.

Furthermore, I would like to thank all members of the Lindenbach lab - Natalie Counihan, Peniel Dimberu, Teymur Kazakov, Chris Peters, Tung Phan and Kenny Stapleford - for their consistent help and scientific advice during the whole time. It was a great pleasure for me to work with you and I am deeply appreciating all the support I got from you.

I am very grateful to the German Academic Exchange Service (DAAD) for supporting me with a scholarship.

I addition I would like to thank my sister Yvonne for her invaluable scientific help and support during this thesis. 


\section{Lebenslauf}

Ich, Caroline Bouter, wurde am 10.05.1987 als Tochter des Diplom-Informatikers Jaap Bouter und der Realschullehrerin Marion Bouter geb. Schrader geboren.

Von 1993 bis 1999 besuchte ich die Grundschule und die Orientierungsstufe Groß Ilsede. Im Anschluss daran ging ich 1999 bis 2006 zum Gymnasium Groß Ilsede und schloss meine schulische Laufbahn mit dem Abitur mit der Note 1,7 ab.

Im April 2007 begann ich mein Studium der Humanmedizin an der Justus-LiebigUniversität in Gießen. Im März 2009 absolvierte ich den Ersten Abschnitt der Ärztlichen Prüfung mit der Note „sehr gut“.

Im April 2009 wechselte ich zum klinischen Studienabschnitt an die Georg-AugustUniversität Göttingen. Im Mai 2010 begann ich mit der experimentellen Arbeit zu der vorliegenden Dissertation an der Yale University in New Haven, CT, USA im Department of Microbial Pathogenesis in der Arbeitsgruppe von Brett Lindenbach unter der Betreuung von Prof. Dr. Frank Hufert aus der Abteilung Virologie der Universität Göttingen. Für meine Zeit in den USA erhielt ich ein Stipendium des Deutschen Akademischen Austauschdienstes. 